\title{
Synthesis of Polycyclic Benzonitriles via a One-Pot Aryl Alkylation/Cyanation Reaction
}

\author{
Brian Mariampillai, Dino Alberico, Valérie Bidau and Mark Lautens* \\ Davenport Research Laboratories, Department of Chemistry, University of Toronto, Toronto, \\ Ontario, Canada M5S 3 H6
}

\section{SUPPORTING INFORMATION}

General Experimental _ S2

Synthesis of Bicyclic Substrates $\quad$ S3

Synthesis of Tricyclic Substrates _ S7

Tandem Ortho Alkylation/Cyanation Reaction __ S12

Modification of Benzonitrile Products __ S17

Spectral Data_ $\quad$ S19 


\section{General Experimental}

Infrared (IR) spectra were recorded on a Perkin Elmer FT-IR spectrometer, SPECTRUM 1000. ${ }^{1} \mathrm{H}$ and ${ }^{13} \mathrm{C}$ NMR spectra were measured on Varian Unity 500, Varian Mercury 400, Varian $400 \mathrm{MHz}$ NMR System, Varian Mercury 300 spectrometer. Splitting patterns are indicated as s, singlet; d, doublet; t, triplet; q, quartet; m, multiplet; br, broad peak. For thin layer chromatography (TLC) analysis throughout this work, Silicycle precoated TLC plates (silica gel $60 \mathrm{~F}_{254}$ on aluminum sheet) were used. The products were purified by preparative column chromatography on silica gel $(60 \AA$, ultrapure silica gel, SILICYCLE). The high-resolution mass spectra (HRMS) analysis was performed at the Department of Chemistry, University of Toronto (EI and ESI). Microwave-assisted reactions were performed in Emrys Liberator model from Personal Chemistry. Reactants were filled into appropriate microwave process vials. Dimethoxyethane was distilled from calcium hydride still under nitrogen prior to use. 3-Iodo-4-methyl-phenol, 3Bromo-4-methyl-phenylamine, 3,5-dimethoxyphenylmagnesium chloride and 2,4,5triiodophenol were purchased from Aldrich and used as received. N-(3-Iodo-4-methylphenyl)-4-methyl-benzenesulfonamide, 3-Iodo-4-methoxy-phenol, and 3-Iodo-4-nitrophenol were made according to literature procedure ${ }^{1,2,3}$ respectively.

\footnotetext{
${ }^{1}$ Bressy, C.; Alberico, D.; Lautens, M. J. Am. Chem.Soc. 2005, 127, 13148-13149.

${ }^{2}$ Hoeger, S. Liebigs Annalen/ Recueil 1997, 1, 273-277.

${ }^{3}$ Shen, Y.; Xie, Q.; Norberg, M.; Sausville, E.; Vande Woude, G.; Wenkert, D. Bioorg. Med. Chem. 2005, 13, 4960-4971.
} 


\section{Synthesis of Bicyclic Substrates}<smiles></smiles>

$\boldsymbol{N}$-(3-Bromo-4-methyl-phenyl)-4-methyl-benzenesulfonamide (28): To a solution of 3-Bromo-4-methyl-phenylamine $(1 \mathrm{~g}, 5.37 \mathrm{mmol})$ in pyridine $(10 \mathrm{~mL})$ was added paratoluenesulfonyl chloride $(1.05 \mathrm{~g}, 5.5 \mathrm{mmol})$ The resulting solution was heated at $70{ }^{\circ} \mathrm{C}$ for 8 hours and the concentrated under reduced pressure, dissolved in dichloromethane and washed with $10 \%$ aqueous hydrochloric acid, brine and dried over magnesium sulfate. The solution was concentrated under reduced pressure to give $1.78 \mathrm{~g}$ ( $95 \%$ yield) of 28 as a pale brown solid, $\mathrm{mp}=139-140{ }^{\circ} \mathrm{C} .{ }^{1} \mathrm{H}$ NMR $\left(\mathrm{CDCl}_{3}, 400 \mathrm{MHz}\right) \delta 7.69$ (d, $J=8.4 \mathrm{~Hz}, 2 \mathrm{H}), 7.38$ (br s, 1H), $7.29(\mathrm{~d}, J=2.2 \mathrm{~Hz}, 1 \mathrm{H}), 7.22(\mathrm{~d}, J=8.0 \mathrm{~Hz}, 2 \mathrm{H}), 7.04$ (d, $J=8.2 \mathrm{~Hz}, 1 \mathrm{H}) 6.96(\mathrm{dd}, J=8.3 \mathrm{~Hz}, J=2.2 \mathrm{~Hz}, 1 \mathrm{H}), 2.37(\mathrm{~s}, 3 \mathrm{H}), 2.28$ (s, 3H) $;{ }^{13} \mathrm{C}-$ NMR $\left(\mathrm{CDCl}_{3}, 100 \mathrm{MHz}\right) 144.1,135.7,135.3,134.8,131.1,129.8,127.3,125.1,124.9$, 120.4, 22.2, 21.6; $\delta$ IR (film) 3250, 2920, 2360, 1603, 1490, 1457, 1374, 1323, 1160, 1091, 1038, 927, $812 \mathrm{~cm}^{-1}$; HRMS-ESI $(\mathrm{m} / \mathrm{z}):\left[\mathrm{M}^{+}\right]$calcd for $\left[\mathrm{C}_{14} \mathrm{H}_{14} \mathrm{NO}_{2} \mathrm{NaSBr}\right]^{+}$: 361.9820, found 361.9838;

General procedure for bicyclic precursors: To the corresponding phenol or tosylated aniline was added 5 equivalents $\mathrm{K}_{2} \mathrm{CO}_{3}$ and 10 equivalents of dibromoalkane $(1 \mathrm{M}$ in acetone). The resulting solution was refluxed under argon for 60 hours. The mixture was the diluted with water and extracted with dichloromethane. The organic phase was dried over magnesium sulfate and evaporated under reduced pressure.<smiles>Cc1ccc(OCCBr)cc1I</smiles>

4-(2-Bromo-ethoxy)-2-iodo-1-methyl-benzene (1): Following the general procedure above on $1 \mathrm{~g}$ ( $4.27 \mathrm{mmol}$ ) of 3-Iodo-4-methyl-phenol, the crude product was purified by column chromatography using $10 \%$ ether in hexanes to give $827 \mathrm{mg}$ (57\% yield) of 1 as a white solid, $\mathrm{mp}=27-28{ }^{\circ} \mathrm{C} .{ }^{1} \mathrm{H} \mathrm{NMR}\left(\mathrm{CDCl}_{3}, 400 \mathrm{MHz}\right) \delta 7.37(\mathrm{~d}, J=2.6 \mathrm{~Hz}, 1 \mathrm{H})$, $7.12(\mathrm{~d}, J=8.4 \mathrm{~Hz}, 1 \mathrm{H}), 6.82(\mathrm{dd}, J=8.4 \mathrm{~Hz}, J=2.6 \mathrm{~Hz}, 1 \mathrm{H}), 4.24(\mathrm{t}, J=7.2 \mathrm{~Hz}, 2 \mathrm{H}), 3.61$ $(\mathrm{t}, J=6.3 \mathrm{~Hz}, 2 \mathrm{H}), 2.37(\mathrm{~s}, 3 \mathrm{H}) ;{ }^{13} \mathrm{C}-\mathrm{NMR} \quad\left(\mathrm{CDCl}_{3}, 100 \mathrm{MHz}\right) \delta 156.3,134.3,129.8$, 125.1, 115.0, 100.8, 68.2, 28.9, 26.9; IR (film) 3057, 3018, 2967, 2917, 2858, 1596, $1565,1486,1453,1420,1380,1287,1234,1076,1016,896,862,841,802,763.688$, $668 \mathrm{~cm}^{-1}$; HRMS-EI (m/z): $\left[\mathrm{M}^{+}\right]$calcd for $\mathrm{C}_{9} \mathrm{H}_{10} \mathrm{BrIO}: 339.8959$, found 339.8960 . 


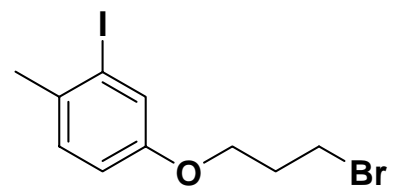

4-(3-Bromo-propoxy)-2-iodo-1-methyl-benzene (3): Following the general procedure above on $1 \mathrm{~g}$ ( $4.27 \mathrm{mmol}$ ) of 3-Iodo-4-methyl-phenol, the crude product was purified by column chromatography using $10 \%$ ether in hexanes to give $992 \mathrm{mg}$ (66\% yield) of 3 as a clear, colorless oil. ${ }^{1} \mathrm{H} \mathrm{NMR}\left(\mathrm{CDCl}_{3}, 400 \mathrm{MHz}\right) \delta 7.39(\mathrm{~d}, J=2.6 \mathrm{~Hz}, 1 \mathrm{H}), 7.12(\mathrm{~d}$, $J=8.2 \mathrm{~Hz}, 1 \mathrm{H}), 6.82(\mathrm{dd}, J=8.3 \mathrm{~Hz}, J=2.6 \mathrm{~Hz}, 1 \mathrm{H}), 4.05(\mathrm{t}, J=5.8 \mathrm{~Hz}, 2 \mathrm{H}), 3.59 \quad$ (t, $J=6.4 \mathrm{~Hz}, 2 \mathrm{H}), 2.38(\mathrm{~s}, 3 \mathrm{H}) 2.34-2.25(\mathrm{~m}, 2 \mathrm{H}) ;{ }^{13} \mathrm{C}-\mathrm{NMR}\left(\mathrm{CDCl}_{3}, 100 \mathrm{MHz}\right) \delta 156.8$, 133.5, 129.7, 124.7, 114.7, 100.9, 65.6, 32.3, 29.9, 26.9; IR (film) 2925, 2920, 1596, $1560,1489,1467,1437,1380,1284,1234,1200,1153,1020,936,856,804,708 \mathrm{~cm}^{-1}$; HRMS-EI (m/z): $\left[\mathrm{M}^{+}\right]$calcd for $\mathrm{C}_{10} \mathrm{H}_{12} \mathrm{BrIO}$ : 353.9116, found 353.9114.<smiles>Cc1ccc(OCCCCBr)cc1I</smiles>

4-(4-Bromo-butoxy)-2-iodo-1-methyl-benzene (5): Following the general procedure above on $1 \mathrm{~g}$ ( $4.27 \mathrm{mmol})$ of 3-Iodo-4-methyl-phenol, the crude product was purified by column chromatography using $10 \%$ ether in hexanes to give $1127 \mathrm{mg}$ ( $72 \%$ yield) of 5 as a clear colorless oil. ${ }^{1} \mathrm{H}$ NMR $\left(\mathrm{CDCl}_{3}, 400 \mathrm{MHz}\right) \delta 7.33(\mathrm{~d}, J=2.6 \mathrm{~Hz}, 1 \mathrm{H}), 7.08$ (d, $J=8.4 \mathrm{~Hz}, 1 \mathrm{H}), 6.77(\mathrm{dd}, J=8.4 \mathrm{~Hz}, J=2.6 \mathrm{~Hz}, 1 \mathrm{H}), 3.91(\mathrm{t}, J=6.0 \mathrm{~Hz}, 2 \mathrm{H}), 3.45 \quad$ (t, $J=6.6 \mathrm{~Hz}, 2 \mathrm{H}), 2.34(\mathrm{~s}, 3 \mathrm{H}) 2.10-1.96(\mathrm{~m}, 2 \mathrm{H}), 1.95-1.84(\mathrm{~m}, 2 \mathrm{H}) ;{ }^{13} \mathrm{C}-\mathrm{NMR}\left(\mathrm{CDCl}_{3}, 100\right.$ MHz) $\delta 157.0,133.3,129.7,124.6,114.6,100.8,67.1,33.4,29.4,27.8,26.9$; IR (film) 2942, 2930, 1596, 1560, 1489, 1469, 1437, 1380, 1285, 1233, 1201, 1021, 946, 908, 861, 812, $732 \mathrm{~cm}^{-1}$; HRMS-EI (m/z): [ $\left.\mathrm{M}^{+}\right]$calcd for $\mathrm{C}_{11} \mathrm{H}_{14} \mathrm{BrIO}$ : 367.9272 , found 367.9278 .<smiles>O=[N+]([O-])c1ccc(OCCBr)cc1I</smiles>

4-(2-Bromo-ethoxy)-2-iodo-1-nitro-benzene (7): Following the general procedure above on $1 \mathrm{~g}$ (3.77 mmol) of 3-Iodo-4-nitro-phenol, the crude product was purified by column chromatography using $10 \%$ ether in hexanes to give $569 \mathrm{mg}$ (41\% yield) of 7 as a bright yellow solid, $\mathrm{mp}=73-75{ }^{\circ} \mathrm{C}$. ${ }^{1} \mathrm{H} \mathrm{NMR}\left(\mathrm{CDCl}_{3}, 400 \mathrm{MHz}\right) \delta 7.95(\mathrm{~d}, J=9.1 \mathrm{~Hz}$, $1 \mathrm{H}), 7.53(\mathrm{~d}, J=2.6 \mathrm{~Hz}, 1 \mathrm{H}), 6.95(\mathrm{dd}, J=9.0 \mathrm{~Hz}, J=2.7 \mathrm{~Hz} 1 \mathrm{H}), 4.35$ (t, $J=6.0 \mathrm{~Hz}, 2 \mathrm{H})$, $3.65(\mathrm{t}, J=6.0 \mathrm{~Hz}, 2 \mathrm{H}) ;{ }^{13} \mathrm{C}-\mathrm{NMR} \quad\left(\mathrm{CDCl}_{3}, 100 \mathrm{MHz}\right) \delta 160.9,145.8,127.7,127.5$, 114.5, 88.2, 68.5, 28.2; IR (film)3628, 2942, 2252, 1587, 1539, 1521, 1453, 1369, 1341, 1278, 1247, 1227, 1014, 908, 850, 734, $650 \mathrm{~cm}^{-1}$; HRMS-EI $(\mathrm{m} / \mathrm{z}):\left[\mathrm{M}^{+}\right]$calcd for $\mathrm{C}_{8} \mathrm{H}_{7} \mathrm{BrINO}_{3}: 370.8654$, found 370.8655 . 
<smiles>COc1ccc(OCCBr)cc1I</smiles>

4-(2-Bromo-ethoxy)-2-iodo-1-methoxy-benzene (9): Following the general procedure above on $1 \mathrm{~g}$ ( $4.00 \mathrm{mmol})$ of 3-Iodo-4-methoxy-phenol, the crude product was purified by column chromatography using $10 \%$ ether in hexanes to give $1135 \mathrm{mg}$ (79\% yield) of 9 as a clear, light yellow oil. ${ }^{1} \mathrm{H}$ NMR $\left(\mathrm{CDCl}_{3}, 400 \mathrm{MHz}\right) \delta 7.36(\mathrm{~d}, J=2.9 \mathrm{~Hz}, 1 \mathrm{H})$, $6.88(\mathrm{dd}, J=8.9 \mathrm{~Hz}, J=2.8 \mathrm{~Hz}, 1 \mathrm{H}), 6.74(\mathrm{~d}, J=8.9 \mathrm{~Hz}, 1 \mathrm{H}), 4.21 \quad(\mathrm{t}, J=6.2 \mathrm{~Hz}, 2 \mathrm{H}), 3.82$ $(\mathrm{s}, 3 \mathrm{H}), 3.59$ (t, $J=6.3 \mathrm{~Hz}, 2 \mathrm{H}) ;{ }^{13} \mathrm{C}-\mathrm{NMR} \quad\left(\mathrm{CDCl}_{3}, 75 \mathrm{MHz}\right) \delta 153.5,152.8,126.4$, 116.1, 111.6, 86.2, 69.1, 57.1, 29.3; IR (film) 2946, 2834, 1598, 2224, 1598, 1572, 1489, 1457, 1437, 1420, 1384, 1271, 1212, 1180, 1077, 1047, 1014, 909, 867, 804, 758, 734, $648 \mathrm{~cm}^{-1}$; HRMS-EI $(\mathrm{m} / \mathrm{z}):\left[\mathrm{M}^{+}\right]$calcd for $\mathrm{C}_{9} \mathrm{H}_{10} \mathrm{BrIO}_{2}: 355.8908$, found 355.8910 .<smiles>Cc1ccc(N(S)CCBr)cc1I</smiles>

\section{$N$-(2-Bromo-ethyl)- $N$-(3-iodo-4-methyl-phenyl)-4-methyl-benzenesulfonamide}

(11a): Following the general procedure above on $1 \mathrm{~g}$ (2.58 mmol) of $\mathrm{N}$-(3-Iodo-4methyl-phenyl)-4-methyl-benzenesulfonamide, the crude product was purified by column chromatography using $20 \%$ ether in hexanes to give $1128 \mathrm{mg}(88 \%$ yield) of $\mathbf{1 1}$ as a white solid, $\mathrm{mp}=.75-76^{\circ} \mathrm{C} .{ }^{1} \mathrm{H} \mathrm{NMR}\left(\mathrm{CDCl}_{3}, 400 \mathrm{MHz}\right) \delta 7.50(\mathrm{~d}, J=8.3 \mathrm{~Hz}, 2 \mathrm{H}), 7.42$ $(\mathrm{d}, J=2.2 \mathrm{~Hz}, 1 \mathrm{H}), 7.29$ (d, $J=8.0 \mathrm{~Hz}, 2 \mathrm{H}), 7.18$ (d, $J=8.1 \mathrm{~Hz}, 1 \mathrm{H}), 6.99$ (dd, $J=7.9 \mathrm{~Hz}$, $J=2.2 \mathrm{~Hz}, 1 \mathrm{H}), 3.81 \quad(\mathrm{t}, J=7.4 \mathrm{~Hz}, 2 \mathrm{H}), 3.38(\mathrm{t}, J=7.4 \mathrm{~Hz}, 2 \mathrm{H}), 2.45(\mathrm{~s}, 3 \mathrm{H}), 2.42 \quad(\mathrm{~s}$, $3 \mathrm{H}) ;{ }^{13} \mathrm{C}-\mathrm{NMR} \quad\left(\mathrm{CDCl}_{3}, 100 \mathrm{MHz}\right) \delta 144.0,142.0,138.9,137.5,134.8,129.8,129.6$, 128.8, 127.7, 100.3, 52.6, 28.7, 27.7, 21.6; IR (film) 3054, 2971, 2919, 1917, 1592, $1556,1481,1445,1349,1304,1275,1250,1220,1032,1017,965,903,814,736,706$,

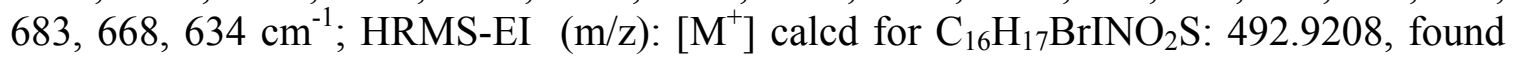
492.9206 .<smiles>Cc1ccc(N([As])CCCBr)cc1I</smiles>

$N$-(3-Bromo-propyl)- $N$-(3-iodo-4-methyl-phenyl)-4-methyl-benzenesulfonamide (13a): Following the general procedure above on $1 \mathrm{~g} \quad(2.58 \mathrm{mmol})$ of $\mathrm{N}$-(3-Iodo-4methyl-phenyl)-4-methyl-benzenesulfonamide, the crude product was purified by column chromatography using 20\% ether in hexanes to give $1227 \mathrm{mg}$ ( $94 \%$ yield) 13 clear colorless oil. ${ }^{1} \mathrm{H} \mathrm{NMR}\left(\mathrm{CDCl}_{3}, 400 \mathrm{MHz}\right) \delta 7.46(\mathrm{~d}, J=7.7 \mathrm{~Hz}, 2 \mathrm{H}), 7.40(\mathrm{~d}, J=1.5 \mathrm{~Hz}$, 
1H), 7.27 (d, $J=8.1 \mathrm{~Hz}, 2 \mathrm{H}), 7.17$ (d, $J=8.1 \mathrm{~Hz}, 1 \mathrm{H}), 6.96$ (dd, $J=8.0 \mathrm{~Hz}, J=1.5 \mathrm{~Hz}, 1 \mathrm{H}$ ), 3.60 (t, $J=6.4 \mathrm{~Hz}, 2 \mathrm{H}), 3.41$ (t, $J=6.5 \mathrm{~Hz}, 2 \mathrm{H}), 2.44(\mathrm{~s}, 3 \mathrm{H}), 2.42$ (s, 3H), 2.05-1.94 (m, $2 \mathrm{H}) ;{ }^{13} \mathrm{C}-\mathrm{NMR}\left(\mathrm{CDCl}_{3}, 100 \mathrm{MHz}\right) \delta 143.8,141.6,138.4,137.6,134.5,129.6,129.5$, 128.4, 127.7, 100.3, 49.1, 31.5, 29.8, 27.6, 21.5; IR (film) 2954, 2943, 2932, 2255, 1744, 1592, 1554, 1482, 1445, 1348, 1304, 1237, 1184, 1165, 1090, 1031, 911, 814, 731, 707, 684, $669 \mathrm{~cm}^{-1}$; HRMS-EI $(\mathrm{m} / \mathrm{z})$ : $\left[\mathrm{M}^{+}\right]$calcd for $\mathrm{C}_{17} \mathrm{H}_{19} \mathrm{BrINO}_{2} \mathrm{~S}$ : 506.9364, found 506.9377 .<smiles>Cc1ccc(N(C)S)cc1I</smiles>

$\mathrm{N}$-(4-Bromo-butyl)- $\mathrm{N}$-(3-iodo-4-methyl-phenyl)-4-methyl-benzenesulfonamide (15a): Following the general procedure above on $1 \mathrm{~g} \quad(2.58 \mathrm{mmol})$ of N- (3-Iodo-4methyl-phenyl)-4-methyl-benzenesulfonamide, the crude product was purified by column chromatography using $20 \%$ ether in hexanes to give $1209 \mathrm{mg}$ (92\% yield) of $\mathbf{1 5}$ as a white solid, $\mathrm{mp}=66-68{ }^{\circ} \mathrm{C} .{ }^{1} \mathrm{H} \mathrm{NMR}\left(\mathrm{CDCl}_{3}, 300 \mathrm{MHz}\right) \delta 7.46(\mathrm{~d}, J=8.3 \mathrm{~Hz}, 2 \mathrm{H}), 7.39$ $(\mathrm{d}, J=2.2 \mathrm{~Hz}, 1 \mathrm{H}), 7.27(\mathrm{~d}, J=8.0 \mathrm{~Hz}, 2 \mathrm{H}), 7.18(\mathrm{~d}, J=8.1 \mathrm{~Hz}, 1 \mathrm{H}), 6.96(\mathrm{dd}, J=8.1 \mathrm{~Hz}$, $J=2.2 \mathrm{~Hz}, 1 \mathrm{H}), 3.49$ (t, $J=7.4 \mathrm{~Hz}, 2 \mathrm{H}), 3.40 \quad(\mathrm{t}, J=7.4 \mathrm{~Hz}, 2 \mathrm{H}), 2.44$ (s, 3H), $2.42 \quad$ (s, $3 \mathrm{H}), 1.92(\mathrm{~m}, 2 \mathrm{H}), 1.55(\mathrm{~m}, 2 \mathrm{H}) ;{ }^{13} \mathrm{C}-\mathrm{NMR}\left(\mathrm{CDCl}_{3}, 75 \mathrm{MHz}\right) \delta 143.9,141.8,138.8$, 137.6, 135.0, 129.9, 129.7, 1.8, 128.0, 100.5, 49.6, 33.3, 29.4, 27.9, 26.5, 21.8; IR (film) 2943, 2255 1740, 1592, 1554, 1477, 1444, 1379, 1348, 1304, 1255, 1184, 1164, 1090, 1030, 910, 814, 707, 684, $669 \mathrm{~cm}^{-1}$; HRMS-EI $(\mathrm{m} / \mathrm{z}):\left[\mathrm{M}^{+}\right]$calcd for $\mathrm{C}_{18} \mathrm{H}_{21} \mathrm{BrINO}_{2} \mathrm{~S}$ : 520.9521 , found 520.9525 .<smiles>Cc1ccc(N(S)CCBr)cc1Br</smiles>

$\mathrm{N}$-(2-Bromo-ethyl)- $\mathrm{N}$-(3-bromo-4-methyl-phenyl)-4-methyl-benzenesulfonamide

(11b): Following the general procedure above on $1 \mathrm{~g}(2.93 \mathrm{mmol})$ of $\mathbf{2 8}$, the crude product was purified by column chromatography using $20 \%$ ether in hexanes to give $1043 \mathrm{mg}(80 \%$ yield $)$ of $\mathbf{1 1 b}$ as a white solid, $\mathrm{mp}=81-82{ }^{\circ} \mathrm{C} .{ }^{1} \mathrm{H} \mathrm{NMR}\left(\mathrm{CDCl}_{3}, 400\right.$ MHz) $\delta 7.49$ (d, $J=8.3 \mathrm{~Hz}, 2 \mathrm{H}), 7.27$ (d, $J=8.2 \mathrm{~Hz}, 2 \mathrm{H}), 7.21(\mathrm{~d}, J=8.0 \mathrm{~Hz}, 1 \mathrm{H}), 7.18$ (d, $J=8.1 \mathrm{~Hz}, 1 \mathrm{H}), 6.93$ (dd, $J=8.1 \mathrm{~Hz}, J=2.2 \mathrm{~Hz}, 1 \mathrm{H}), 3.82$ (t, $J=7.4 \mathrm{~Hz}, 2 \mathrm{H}), 3.37$ (t, $J=7.4 \mathrm{~Hz}, 2 \mathrm{H}), 2.43(\mathrm{~s}, 3 \mathrm{H}), 2.38(\mathrm{~s}, 3 \mathrm{H}) ;{ }^{13} \mathrm{C}-\mathrm{NMR}\left(\mathrm{CDCl}_{3}, 75 \mathrm{MHz}\right) \delta 144.2,138.7$, 137.9, 135.0, 132.7, 131.2, 129.8, 128.0, 127.9, 124.9, 52.7, 28.9, 22.8, 21.8; IR (film) 3025, 2922, 2256, 1597, 1597, 1560, 1487, 1447, 1350, 1305, 1250, 1222, 1160, 1089, 1038, 1018, 965, 908, 804, 732, 707, 668, 635, $601 \mathrm{~cm}^{-1}$; HRMS-EI (m/z): $\left[\mathrm{M}^{+}\right]$calcd for $\mathrm{C}_{16} \mathrm{H}_{17} \mathrm{Br}_{2} \mathrm{NO}_{2} \mathrm{~S}$ : 444.9346, found 444.9350 . 
<smiles>Cc1ccc(N([As])CCCBr)cc1Br</smiles>

$N$-(3-Bromo-4-methyl-phenyl)- $N$-(3-bromo-propyl)-4-methyl-benzenesulfonamide (13b): Following the general procedure above on $0.5 \mathrm{~g}(1.46 \mathrm{mmol})$ of $\mathbf{2 8}$, the crude product was purified by column chromatography using $20 \%$ ether in hexanes to give 608 mg $\left(89 \%\right.$ yield) of $\mathbf{1 3 b}$ as a thick colourless oil. ${ }^{1} \mathrm{H} \mathrm{NMR}\left(\mathrm{CDCl}_{3}, 400 \mathrm{MHz}\right) \delta 7.47$ (d, $J=8.3 \mathrm{~Hz}, 2 \mathrm{H}), 7.27(\mathrm{~d}, J=10.3 \mathrm{~Hz}, 2 \mathrm{H}), 7.17-7.19(\mathrm{~m}, 2 \mathrm{H}), 6.91 \quad(\mathrm{dd}, J=7.9 \mathrm{~Hz}, J=2.2$ $\mathrm{Hz}, 1 \mathrm{H}), 3.62(\mathrm{t}, J=6.7 \mathrm{~Hz}, 2 \mathrm{H}), 3.42(\mathrm{t}, J=6.7 \mathrm{~Hz}, 2 \mathrm{H}), 2.44(\mathrm{~s}, 3 \mathrm{H}), 2.38(\mathrm{~s}, 3 \mathrm{H}), 2.00$ $(\mathrm{m}, 2 \mathrm{H}) ;{ }^{13} \mathrm{C}-\mathrm{NMR} \quad\left(\mathrm{CDCl}_{3}, 100 \mathrm{MHz}\right) \delta 143.8,138.1,137.8,134.4,132.0,130.9$, 129.5, 127.7, 127.4, 124.6, 49.0, 31.4, 29.8, 22.5, 21.5; IR (film) 2921, 2865, 2361 1733, 1597, 1558, 1489, 1349, 1304, 1238, 1184, 1163, 1090, 1037, 913, 814, 707, 694, 676, 652, $605 \mathrm{~cm}^{-1}$; HRMS-EI $(\mathrm{m} / \mathrm{z}):\left[\mathrm{M}^{+}\right]$calcd for $\mathrm{C}_{17} \mathrm{H}_{20} \mathrm{Br}_{2} \mathrm{NO}_{2} \mathrm{~S}: 459.9575$, found 459.9564 .<smiles>Cc1ccc(N(C)S)cc1Br</smiles>

$\mathrm{N}$-(4-Bromo-butyl)- $\mathrm{N}$-(3-bromo-4-methyl-phenyl)-4-methyl-benzenesulfonamide (15b): Following the general procedure above on $0.5 \mathrm{~g}(1.46 \mathrm{mmol})$ of $\mathbf{2 8}$, the crude product was purified by column chromatography using $20 \%$ ether in hexanes to give 640 mg $\left(92 \%\right.$ yield) of $\mathbf{1 5 b}$ as a white solid, $\mathrm{mp}=67-69^{\circ} \mathrm{C} .{ }^{1} \mathrm{H} \mathrm{NMR}\left(\mathrm{CDCl}_{3}, 400 \mathrm{MHz}\right) \delta$ $7.46(\mathrm{~d}, J=8.3 \mathrm{~Hz}, 2 \mathrm{H}), 7.27$ (d, $J=7.2 \mathrm{~Hz}, 2 \mathrm{H}), 7.18(\mathrm{~m}, 2 \mathrm{H}), 6.92$ (dd, $J=8.1 \mathrm{~Hz}, J=2.0$ $\mathrm{Hz}, 1 \mathrm{H}), 3.50$ (t, $J=6.8 \mathrm{~Hz}, 2 \mathrm{H}), 3.40$ (t, $J=6.7 \mathrm{~Hz}, 2 \mathrm{H}), 2.43(\mathrm{~s}, 3 \mathrm{H}), 2.38(\mathrm{~s}, 3 \mathrm{H}), 1.93$ $(\mathrm{m}, 2 \mathrm{H}), 1.55(\mathrm{~m}, 2 \mathrm{H}) ;{ }^{13} \mathrm{C}-\mathrm{NMR}\left(\mathrm{CDCl}_{3}, 100 \mathrm{MHz}\right) \delta 143.9,138.2,137.7,134.8,132.3$, 131.1, 129.7, 127.9, 127.8, 124.7, 49.4, 33.3, 29.3, 26.4, 22.8, 21.8; IR (film) 3027, 2922, 2867, 2255, 1917, 1734, 1597, 1558, 1489, 1447, 1379, 1348, 1304, 1286, 1255, 1230, 1183, 1162, 1090, 1037, 927, 814, 735, 707, 694, 675, 652, $600 \mathrm{~cm}^{-1}$; HRMS-EI $(\mathrm{m} / \mathrm{z})$ : $\left[\mathrm{M}^{+}\right]$calcd for $\mathrm{C}_{18} \mathrm{H}_{22} \mathrm{Br}_{2} \mathrm{NO}_{2} \mathrm{~S}: 473.9732$, found 473.9721 .

\section{Synthesis of Tricyclic Substrates}<smiles>COc1cc(I)cc(OC)c1</smiles>

1-Iodo-3,5-dimethoxybenzene (29): To a solution of 3,5-dimethoxyphenylmagnesium chloride $(50.0 \mathrm{~mL}, 50.0 \mathrm{mmol}, 1 \mathrm{M}$ in THF, 1 equiv) was added drop wise a solution of iodine $(8.38 \mathrm{~g}, 33.0 \mathrm{~mL}, 0.66$ equiv) in THF $(30 \mathrm{~mL})$. The resulting mixture was stirred 
at $0{ }^{\circ} \mathrm{C}$ for $2 \mathrm{~h}$ then quenched with $1 \mathrm{M}$ aqueous $\mathrm{HCl}(30 \mathrm{~mL})$. Ether $(25 \mathrm{~mL})$ was added and the organic layer was separated and washed with $1 \mathrm{M}$ aqueous $\mathrm{Na}_{2} \mathrm{~S}_{2} \mathrm{O}_{3}$ solution $(2 \times$ $30 \mathrm{~mL}$ ). After drying with anhydrous Magnesium sulfate, removal of the solvent gave a crude solid that was purified by column chromatography using $10 \%$ EtOAc in hexanes to give $11 \mathrm{~g}$ (93\% yield) of 30 as a white solid, $\mathrm{mp}=74-75^{\circ} \mathrm{C} .{ }^{1} \mathrm{H}$ NMR $(300 \mathrm{MHz}$, $\left.\mathrm{CDCl}_{3}\right) \delta 6.85(\mathrm{~d}, 2 \mathrm{H}, J=2.2 \mathrm{~Hz}), 6.40(\mathrm{t}, 1 \mathrm{H}, J=2.2 \mathrm{~Hz}), 3.76(\mathrm{~s}, 6 \mathrm{H}) ;{ }^{13} \mathrm{C} \mathrm{NMR}(75$ $\mathrm{MHz}, \mathrm{CDCl}_{3}$ ) $\delta 161.2,116.0,100.8,94.3,55.7$; IR (film) 1577, 1425, 1294, 1199, 1164 , $1032 \mathrm{~cm}^{-1}$; HRMS (m/z) : calcd for $\mathrm{C}_{8} \mathrm{H}_{9} \mathrm{IO}_{2}[\mathrm{M}]^{+} 263.9654$, found 263.9647 .<smiles>Oc1cc(O)cc(I)c1</smiles>

5-Iodobenzene-1,3-diol (30): A solution of 29 ( $8.30 \mathrm{~g}, 31.4 \mathrm{mmol}, 1$ equiv) and HI (47 wt $\%$ in water, $75 \mathrm{~mL}$ ) was heated at reflux for $24 \mathrm{~h}$. The solution was cooled to $\mathrm{rt}$ and water $(100 \mathrm{~mL})$ and ether $(100 \mathrm{~mL})$ were added. The organic layer was separated and washed with $1 \mathrm{M}$ sodium thiosulfate solution $(2 \times 75 \mathrm{~mL})$ and once with water $(50 \mathrm{~mL})$. After drying with anhydrous Magnesium sulfate, removal of the solvent gave a crude oil that was purified by column chromatography using 25\% EtOAc in hexanes to give 6.90 $\mathrm{g}(97 \%$ yield $)$ of 30 as a white solid, $\mathrm{mp}=94-95{ }^{\circ} \mathrm{C} .{ }^{1} \mathrm{H}$ NMR $\left(300 \mathrm{MHz}\right.$, acetone- $\left.d_{6}\right) \delta$ 8.40 (br s, 2OH), $6.33(\mathrm{~s}, 2 \mathrm{H}), 6.72(\mathrm{~s}, 1 \mathrm{H}) ;{ }^{13} \mathrm{C}$ NMR $\left(75 \mathrm{MHz}\right.$, acetone- $\left.d_{6}\right) \delta 159.3$, 116.3, 106.8, 93.8; IR (film) 3330, 1591, 1470, 1294, 1152, $\mathrm{cm}^{-1} ;$ HRMS (m/z): calcd for $\mathrm{C}_{6} \mathrm{H}_{5} \mathrm{IO}_{2}[\mathrm{M}]^{+}$235.9334, found 235.9334 .<smiles>BrCCOc1cc(I)cc(OCCBr)c1</smiles>

1,3-Bis-(2-bromo-ethoxy)-5-iodo-benzene (17): To a solution of 30 (8.00 g, 33.9 mmol, 1 equiv) and 1,2-dibromoethane $(44.0 \mathrm{~mL}, 508 \mathrm{mmol}, 15$ equiv) in acetone (70 $\mathrm{mL}$ ) was added $\mathrm{K}_{2} \mathrm{CO}_{3}$ (23.4 g, $170 \mathrm{mmol}, 5$ equiv). The mixture was heated at reflux for $36 \mathrm{~h}$. The reaction was cooled to $\mathrm{rt}$ and filtered through Celite ${ }^{\mathrm{TM}}$. Distillation of the excess 1,2-dibromoethane gave a crude oil that was purified by column chromatography using 10\% EtOAc in hexanes to give $9.3 \mathrm{~g}$ (62\% yield) of 17 as a white solid, $\mathrm{mp}=97$ $98{ }^{\circ} \mathrm{C} .{ }^{1} \mathrm{H} \mathrm{NMR}\left(300 \mathrm{MHz}, \mathrm{CDCl}_{3}\right) \delta 6.89(\mathrm{~s}, 2 \mathrm{H}), 6.44(\mathrm{~s}, 1 \mathrm{H}), 4.24(\mathrm{t}, 4 \mathrm{H}, J=6.0$ $\mathrm{Hz}), 3.61(\mathrm{t}, 4 \mathrm{H}, J=6.0 \mathrm{~Hz}) ;{ }^{13} \mathrm{C} \mathrm{NMR}\left(75 \mathrm{MHz}, \mathrm{CDCl}_{3}\right) \delta 159.8,117.4,102.3,94.3$, 68.3, 28.9; IR (film) 1421, 1168, $1052 \mathrm{~cm}^{-1}$; HRMS (m/z): calcd for $\mathrm{C}_{10} \mathrm{H}_{11} \mathrm{Br}_{2} \mathrm{IO}_{2}[\mathrm{M}]^{+}$ 447.8161 , found 447.8170 . 
<smiles>BrCCCOc1cc(I)cc(OCCCBr)c1</smiles>

1,3-Bis-(3-bromo-propoxy)-5-iodo-benzene (19): To a solution of 30 (1.24 g, 5.25 mmol, 1 equiv) and 1,3-dibromopropane $(5.30 \mathrm{~mL}, 52.5 \mathrm{mmol}, 10$ equiv) in acetone (15 $\mathrm{mL}$ ) was added $\mathrm{K}_{2} \mathrm{CO}_{3}$ (3.63 g, $26.3 \mathrm{mmol}, 5$ equiv). The mixture was heated at reflux for $36 \mathrm{~h}$. The reaction was cooled to $\mathrm{rt}$ and filtered through Celite ${ }^{\mathrm{TM}}$. Distillation of the excess 1,3-dibromopropane gave a crude oil that was purified by column chromatography using 10\% EtOAc in hexanes to give $1.5 \mathrm{~g}$ (60\% yield) of 19 as a white solid, $\mathrm{mp}=62-63{ }^{\circ} \mathrm{C} .{ }^{1} \mathrm{H} \mathrm{NMR}\left(300 \mathrm{MHz}, \mathrm{CDCl}_{3}\right) \delta 6.87(\mathrm{~d}, 2 \mathrm{H}, J=2.2 \mathrm{~Hz}), 6.42$ (t, $1 \mathrm{H}, J=2.2 \mathrm{~Hz}), 4.06(\mathrm{t}, 4 \mathrm{H}, J=5.8 \mathrm{~Hz}), 3.58(\mathrm{t}, 4 \mathrm{H}, J=6.4 \mathrm{~Hz}), 2.29$ (quintet, $4 \mathrm{H}, J=$ $0.015 \mathrm{~Hz}) ;{ }^{13} \mathrm{C} \mathrm{NMR}\left(75 \mathrm{MHz}, \mathrm{CDCl}_{3}\right) \delta 116.8,160.3,116.8,101.8,94.3,65.8,32.4$, 29.9; IR (film) 1593, 1434, 1166, $1053 \mathrm{~cm}^{-1}$; HRMS (m/z): calcd for $\mathrm{C}_{12} \mathrm{H}_{15} \mathrm{Br}_{2} \mathrm{IO}_{2}[\mathrm{M}]^{+}$ 475.8477, found 475.8483 .<smiles>Oc1cc(I)cc(OCCBr)c1</smiles>

3-(2-Bromoethoxy)-5-iodophenol (31) : To a solution of 30 (2.75 g, $11.6 \mathrm{mmol}, 1$ equiv) and 1,2-dibromoethane $(7.0 \mathrm{~mL}, 81.6 \mathrm{mmol}, 7$ equiv) in acetone $(20 \mathrm{~mL})$ was added $\mathrm{K}_{2} \mathrm{CO}_{3}$ ( $2.10 \mathrm{~g}, 15.1 \mathrm{mmol}, 1.3$ equiv). The mixture was heated at reflux for $24 \mathrm{~h}$. The reaction was cooled to $\mathrm{rt}$ and filtered through Celite ${ }^{\mathrm{TM}}$. Distillation of the excess 1,2dibromoethane gave a crude oil that was purified by column chromatography using $10 \%$ EtOAc in hexanes to give $1.3 \mathrm{~g}$ (34\% yield) of 31 as a white solid, $\mathrm{mp}=139-141{ }^{\circ} \mathrm{C} .{ }^{1} \mathrm{H}$ NMR (400 MHz, $\left.\mathrm{CDCl}_{3}\right) \delta 6.83(\mathrm{~m}, 2 \mathrm{H}), 6.36(\mathrm{t}, 1 \mathrm{H}, J=2.2 \mathrm{~Hz}), 4.89$ (br s, 1OH), $4.21(\mathrm{t}, 2 \mathrm{H}, J=6.2 \mathrm{~Hz}), 3.59(\mathrm{t}, 2 \mathrm{H}, J=6.2 \mathrm{~Hz}) ;{ }^{13} \mathrm{C} \mathrm{NMR}\left(75 \mathrm{MHz}, \mathrm{CDCl}_{3}\right) \delta 159.8$, 157.2, 118.3, 116.8, 102.6, 94.3, 68.2, 28.9; IR (film) 3385, 1596, 1493, 1279, $1148 \mathrm{~cm}^{-}$ ; $\mathrm{HRMS}(\mathrm{m} / \mathrm{z})$ : calcd for $\mathrm{C}_{8} \mathrm{H}_{8} \mathrm{BrIO}_{2}[\mathrm{M}]^{+}$341.8752, found 341.8752 .<smiles>BrCCCOc1cc(I)cc(OCCBr)c1</smiles>

1-(2-Bromoethoxy)-3-(3-bromopropoxy)-5-iodobenzene (21): To a solution of 31 (1.88 g, $5.48 \mathrm{mmol}, 1$ equiv) and 1,3-dibromopropane (5.6 mL, $54.8 \mathrm{mmol}, 10$ equiv) in acetone $(15 \mathrm{~mL})$ was added $\mathrm{K}_{2} \mathrm{CO}_{3}(2.27 \mathrm{~g}, 16.4 \mathrm{mmol}, 3$ equiv). The mixture was heated at reflux for $36 \mathrm{~h}$. The reaction was cooled to $\mathrm{rt}$ and filtered through Celite ${ }^{\mathrm{TM}}$. Distillation of the excess 1,3-dibromopropane gave a crude oil that was purified by column chromatography using 10\% EtOAc in hexanes to give $1.0 \mathrm{~g}$ (40\% yield) of 21 as 
a white solid, $\mathrm{mp}=63-64{ }^{\circ} \mathrm{C} .{ }^{1} \mathrm{H}$ NMR $\left(400 \mathrm{MHz}, \mathrm{CDCl}_{3}\right) \delta 6.90 \quad(\mathrm{~m}, 1 \mathrm{H}), 6.86$ (m, $1 \mathrm{H}), 6.43(\mathrm{t}, 1 \mathrm{H}, J=2.7 \mathrm{~Hz}), 4.24(\mathrm{t}, 2 \mathrm{H}, J=6.2 \mathrm{~Hz}), 4.06(\mathrm{t}, 2 \mathrm{H}, J=6.2 \mathrm{~Hz}), 3.61(\mathrm{t}$, $2 \mathrm{H}, J=2.7 \mathrm{~Hz}$ ), $3.56(\mathrm{t}, 2 \mathrm{H}, J=6.2 \mathrm{~Hz}), 2.29$ (quint, $2 \mathrm{H}, J=6.2 \mathrm{~Hz}) ;{ }^{13} \mathrm{C} \mathrm{NMR}(100$ $\left.\mathrm{MHz}, \mathrm{CDCl}_{3}\right) \delta 160.4,159.7,117.4,116.9,102.1,94.3,68.2,65.8,32.3,29.9,28.9$; IR (film) 1574, 1434, 1277, $1163 \mathrm{~cm}^{-1}$; $\mathrm{HRMS}\left(\mathrm{m} / \mathrm{z}\right.$ ): calcd for $\mathrm{C}_{11} \mathrm{H}_{13} \mathrm{Br}_{2} \mathrm{IO}_{2}[\mathrm{M}]^{+} 461.8333$, found 461.8327.<smiles>COc1c(I)cc(I)cc1I</smiles>

1,3,5-Triiodo-2-methoxybenzene (32): To a solution of 2,4,5-triiodophenol (10.0 g, $21.2 \mathrm{mmol}, 1$ equiv) in acetone $(175 \mathrm{~mL})$ was added $\mathrm{K}_{2} \mathrm{CO}_{3}(4.40 \mathrm{~g}, 31.8 \mathrm{mmol}, 1.5$ equiv). The mixture was stirred at $\mathrm{rt}$ for $30 \mathrm{~min}$ then iodomethane $(2.00 \mathrm{~mL}, 31.8 \mathrm{mmol}$, 1.5 equiv) was added. The mixture was heated at reflux for $3 \mathrm{~h}$, cooled to rt and filtered through Celite ${ }^{\mathrm{TM}}$. Evaporation of the volatiles gave a crude product that was purified by column chromatography using $10 \%$ EtOAc in hexanes to give $10 \mathrm{~g}$ (98\% yield) of 32 as a pale yellow solid, $\mathrm{mp}=93-94{ }^{\circ} \mathrm{C} .{ }^{1} \mathrm{H} \mathrm{NMR}\left(300 \mathrm{MHz}, \mathrm{CDCl}_{3}\right) \delta 8.05(\mathrm{~s}, 2 \mathrm{H}), 3.84(\mathrm{~s}$, $3 \mathrm{H}) ;{ }^{13} \mathrm{C} \mathrm{NMR}\left(75 \mathrm{MHz}, \mathrm{CDCl}_{3}\right) \delta 159.3,147.5,91.9,89.5,61.0$; IR (film) 1455, 1402 $\mathrm{cm}^{-1}$; HRMS (m/z): calcd for $\mathrm{C}_{7} \mathrm{H}_{5} \mathrm{I}_{3} \mathrm{O}[\mathrm{M}]^{+}$485.7481, found 485.7474.<smiles>COc1c(O)cc(I)cc1O</smiles>

5-Iodo-2-methoxybenzene-1,3-diol (33): To a $-78{ }^{\circ} \mathrm{C}$ solution of 32 (8.61 g, 17.7 mmol, 1 equiv) in ether $(165 \mathrm{~mL})$ was added drop wise $n$-BuLi $(24.4 \mathrm{~mL}, 38.9 \mathrm{mmol}$, 1.6 $\mathrm{M}$ in hexanes, 2.2 equiv). The mixture was stirred at $-78{ }^{\circ} \mathrm{C}$ for $1 \mathrm{~h}$ then trimethyl borate $(20.0 \mathrm{~mL}, 17.7 \mathrm{mmol}, 10$ equiv) was added at once. The reaction was warmed to $\mathrm{rt}$ over $16 \mathrm{~h}$ then cooled to $0^{\circ} \mathrm{C}$. Peracetic acid solution $(12.0 \mathrm{~mL}, 17.7 \mathrm{mmol}, 32 \mathrm{wt} \%$ in dilute acetic acid, 10 equiv) was added drop wise. The reaction was stirred at $0{ }^{\circ} \mathrm{C}$ for 30 min then quenched with saturated aqueous $\mathrm{NaHCO}_{3}(12 \mathrm{~mL})$ and then warmed to rt. Water $(170 \mathrm{~mL})$ and ether $(170 \mathrm{~mL})$ were added and the organic layer was washed with saturated aqueous $\mathrm{Na}_{2} \mathrm{~S}_{2} \mathrm{O}_{3}$, brine, dried with Magnesium sulfate, filtered, and concentrated. The crude mixture was purified by column chromatography using $25 \%$ EtOAc in hexanes to give $3.6 \mathrm{~g}$ (77\% yield) of 33 as a pale yellow solid, $\mathrm{mp}=90-91{ }^{\circ} \mathrm{C}$. ${ }^{1} \mathrm{H}$ NMR $\left(300 \mathrm{MHz}, \mathrm{CDCl}_{3}\right) \delta 6,87$ (s, 2H), 5.93 (br s, 2OH), 3.85 (s, 3H); ${ }^{13} \mathrm{C} \mathrm{NMR}$ $\left(75 \mathrm{MHz}, \mathrm{CDCl}_{3}\right) \delta 149.8,135.0,117.9,87.2$, 61.4; IR (film) 3395, 1582, 1487, 1161 $\mathrm{cm}^{-1}$; HRMS (m/z): calcd for $\mathrm{C}_{7} \mathrm{H}_{7} \mathrm{IO}_{3}[\mathrm{M}]^{+} 265.9439$, found 265.9440 . 
<smiles>COc1c(OCCBr)cc(I)cc1OCCBr</smiles>

1,3-Bis(2-bromoethoxy)-5-iodo-2-methoxybenzene (23): To a solution of 33 (4.00 g, $19.4 \mathrm{mmol}, 1$ equiv) and 1,2-dibromoethane $(25.0 \mathrm{~mL}, 291 \mathrm{mmol}, 15$ equiv) in acetone (35 mL) was added $\mathrm{K}_{2} \mathrm{CO}_{3}$ (13.5 g, $97.1 \mathrm{mmol}, 5$ equiv). The mixture was heated at reflux for $36 \mathrm{~h}$. The reaction was cooled to $\mathrm{rt}$ and filtered through Celite ${ }^{\mathrm{TM}}$. Distillation of the excess 1,2-dibromoethane gave a crude oil that was purified by column chromatography using 10\% EtOAc in hexanes to give $7.8 \mathrm{~g}$ (84\% yield) of 23 as a white solid, $\mathrm{mp}=82-83{ }^{\circ} \mathrm{C} .{ }^{1} \mathrm{H} \mathrm{NMR}\left(300 \mathrm{MHz}, \mathrm{CDCl}_{3}\right) \delta 6.92(\mathrm{~s}, 2 \mathrm{H}), 4.24 \quad(\mathrm{t}, 4 \mathrm{H}, J=6.1$ $\mathrm{Hz}), 3.87$ (s, 3H), 3.65 (t, $4 \mathrm{H}, J=6.1 \mathrm{~Hz}) ;{ }^{13} \mathrm{C} \mathrm{NMR}\left(75 \mathrm{MHz}, \mathrm{CDCl}_{3}\right) \delta 153.1,140.1$, 118.3, 85.9, 69.6, 61.5, 29.2; IR (film) 1581, 1496, 1417, $1128 \mathrm{~cm}^{-1}$; HRMS (m/z): calcd for $\mathrm{C}_{11} \mathrm{H}_{13} \mathrm{Br}_{2} \mathrm{IO}_{3}[\mathrm{M}]^{+} 477.8283$, found 477.8276 . 


\section{Tandem Ortho Alkylation/Cyanation Reaction}

General Procedure for Cyanation Reaction: To a $5 \mathrm{ml}$ microwave vial containing a magnetic stir bar was added 1 equivalent substrate, 0.1 equivalents palladium acetate, 0.22 equivalents triphenylphosphine, 1 equivalent zinc cyanide, 3 equivalents norbornene and 2 equivalents cesium carbonate. The vial was the sealed and flushed with argon. Dimethoxyethane was added to give a final substrate concentration of $0.05 \mathrm{M}$. The resulting solution was then purged with argon for 5 minutes followed by microwave irradiation at $150{ }^{\circ} \mathrm{C}$ for 4000 seconds. The pressure noted at this temperature was 5.5 bars. The mixture was then diluted with water and extracted with dichloromethane. The combined organic phases were dried with magnesium sulphate and evaporated under reduced pressure.<smiles>Cc1ccc2c(c1C#N)CCO2</smiles>

5-Methyl-2,3-dihydro-benzofuran-4-carbonitrile (2): Following the general procedure above on $0.2 \mathrm{mmol}$ of $\mathbf{1}$, the crude product was purified by column chromatography using $10 \%$ ether in hexanes to give $29.0 \mathrm{mg}$ (91\% Yield) of 2 as a white solid, $\mathrm{mp}=46$ $48{ }^{\circ} \mathrm{C}$. Alternatively the sealed vial could be placed in a $150{ }^{\circ} \mathrm{C}$ preheated oil bath for 4000 seconds to give $24.5 \mathrm{mg}$ (77\% yield) of $2 .{ }^{1} \mathrm{H} \mathrm{NMR}\left(\mathrm{CDCl}_{3}, 400 \mathrm{MHz}\right) \delta 7.02(\mathrm{~d}$, $J=8.2 \mathrm{~Hz}, 1 \mathrm{H}), 6.86(\mathrm{~d}, J=8.2 \mathrm{~Hz}, 1 \mathrm{H}), 4.64(\mathrm{t}, J=8.8 \mathrm{~Hz}, 2 \mathrm{H}), 3.37$ (t, $J=8.8 \mathrm{~Hz}, 2 \mathrm{H})$, $2.44(\mathrm{~s}, 3 \mathrm{H}) ;{ }^{13} \mathrm{C}-\mathrm{NMR}\left(\mathrm{CDCl}_{3}, 75 \mathrm{MHz}\right) \delta 158.3,133.1,131.5,129.6,116.6,113.5$, 109.3, 71.5, 29.7, 19.5, IR (film) 2960, 2224, 1739, 1605, 1480, 1470, 1438, 1144, 1035, 976, 945, $820 \mathrm{~cm}^{-1}$; HRMS-EI (m/z): $\left[\mathrm{M}^{+}\right]$calcd for $\mathrm{C}_{10} \mathrm{H}_{9} \mathrm{NO}: 159.0684$, found 159.0688 .<smiles>Cc1ccc2c(c1C#N)CCCO2</smiles>

6-Methyl-chroman-5-carbonitrile (4): Following the general procedure above on 0.2 mmol of 3 , the crude product was purified by column chromatography using $10 \%$ ether in hexanes to give $27.0 \mathrm{mg}$ ( $78 \%$ Yield) of 4 as a white solid, $\mathrm{mp}=34-35^{\circ} \mathrm{C} .{ }^{1} \mathrm{H}$ NMR $\left(\mathrm{CDCl}_{3}, 400 \mathrm{MHz}\right) \delta 7.00(\mathrm{~d}, J=8.4 \mathrm{~Hz}, 1 \mathrm{H}), 6.90(\mathrm{~d}, J=8.4 \mathrm{~Hz}, 1 \mathrm{H}), 4.19-4.15(\mathrm{~m}, 2 \mathrm{H})$, $2.94(\mathrm{t}, J=6.6 \mathrm{~Hz}, 2 \mathrm{H}), 2.45(\mathrm{~s}, 3 \mathrm{H}), 2.09-2.02(\mathrm{~m}, 2 \mathrm{H}) ;{ }^{13} \mathrm{C}-\mathrm{NMR}\left(\mathrm{CDCl}_{3}, 100 \mathrm{MHz}\right) \delta$ 153.0, 134.0, 128.6, 125.2, 121.2, 116.7, 113.2, 66.1, 23.9, 21.6, 19.8; IR (film) 2950, 2871, 2219, 1739, 1601, 1485, 1442, 1384, 1365, 1344, 1299, 1257, 1208, 1161, 1089, 1068, 956, 893, 858, 822, $739 \mathrm{~cm}^{-1}$; HRMS-EI $(\mathrm{m} / \mathrm{z}):\left[\mathrm{M}^{+}\right]$calcd for $\mathrm{C}_{11} \mathrm{H}_{11} \mathrm{NO}$ : 173.0840, found 173.0842 . 
<smiles>Cc1ccc2c(c1C#N)CCCCO2</smiles>

7-Methyl-2,3,4,5-tetrahydro-benzo[b]oxepine-6-carbonitrile (6): Following the general procedure above on $0.2 \mathrm{mmol}$ of 5 , the crude product was purified by column chromatography using $10 \%$ ether in hexanes to give $23.0 \mathrm{mg}(62 \%$ Yield) of 6 as a clear colorless oil. ${ }^{1} \mathrm{H} \mathrm{NMR}\left(\mathrm{CDCl}_{3}, 400 \mathrm{MHz}\right) \delta 7.08(\mathrm{~d}, J=8.2 \mathrm{~Hz}, 1 \mathrm{H}), 7.03(\mathrm{~d}, J=8.2 \mathrm{~Hz}$, $1 \mathrm{H}), 4.00-3.96(\mathrm{~m}, 2 \mathrm{H}), 3.1-3.07(\mathrm{~m}, 2 \mathrm{H}), 2.48(\mathrm{~s}, 3 \mathrm{H}), 2.02-1.96(\mathrm{~m}, 2 \mathrm{H}), 1.80-1.73$ $(\mathrm{m}, 2 \mathrm{H}) ;{ }^{13} \mathrm{C}-\mathrm{NMR}\left(\mathrm{CDCl}_{3}, 75 \mathrm{MHz}\right) \delta 158.6,139.2,137.2,128.7,125.7,117.2,113.6$, 73.8, 32.0, 31.8, 25.3, 20.4; IR (film) 2936, 2869, 2220, 1734, 1609, 1576, 1480, 1383,

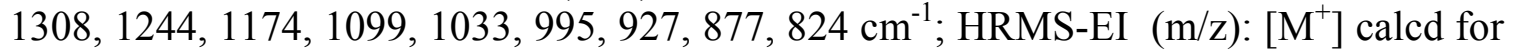
$\mathrm{C}_{12} \mathrm{H}_{13} \mathrm{NO}$ : 187.0997, found 187.0995.<smiles>N#Cc1c([N+](=O)[O-])ccc2c1CCO2</smiles>

5-Nitro-2,3-dihydro-benzofuran-4-carbonitrile (8): Following the general procedure above on $0.2 \mathrm{mmol}$ of 7 , the crude product was purified by column chromatography using $20 \%$ ether in hexanes to give $27.5 \mathrm{mg}(73 \%$ Yield) of 8 as a yellow solid, $\mathrm{mp}=$ 144-145 ${ }^{\circ} \mathrm{C} .{ }^{1} \mathrm{H}$ NMR $\left(\mathrm{CDCl}_{3}, 400 \mathrm{MHz}\right) \delta 8.22(\mathrm{~d}, J=8.9 \mathrm{~Hz}, 1 \mathrm{H}), 7.03(\mathrm{~d}, J=8.9 \mathrm{~Hz}$, $1 \mathrm{H}), 5.87$ (t, $J=8.8 \mathrm{~Hz}, 2 \mathrm{H}), 3.54$ (t, $J=8.8 \mathrm{~Hz}, 2 \mathrm{H}) ;{ }^{13} \mathrm{C}-\mathrm{NMR}\left(\mathrm{CDCl}_{3}, 100 \mathrm{MHz}\right) \delta$ 164.9, 141.2, 136.0, 127.8, 113.6, 112.4, 105.9, 73.4, 29.3; IR (film) 2978, 2279, 1586, 1526, 1340, 993, 981, $807 \mathrm{~cm}^{-1}$; HRMS-EI (m/z): $\left[\mathrm{M}^{+}\right]$calcd for $\mathrm{C}_{9} \mathrm{H}_{6} \mathrm{~N}_{2} \mathrm{O}_{3}: 19.0378$, found 190.0379 .<smiles>COc1ccc2c(c1C#N)CCO2</smiles>

5-Methoxy-2,3-dihydro-benzofuran-4-carbonitrile (10): Following the general procedure above on $0.2 \mathrm{mmol}$ of $\mathbf{9}$, the crude product was purified by column chromatography using $10 \%$ ether in hexanes to give $29.0 \mathrm{mg}(83 \%$ Yield) of $\mathbf{1 0}$ as a white solid, $\mathrm{mp}=131-133{ }^{\circ} \mathrm{C} .{ }^{1} \mathrm{H} \mathrm{NMR}\left(\mathrm{CDCl}_{3}, 400 \mathrm{MHz}\right) \delta 6.90(\mathrm{~d}, J=8.8 \mathrm{~Hz}, 1 \mathrm{H})$, $6.68(\mathrm{~d}, J=8.8 \mathrm{~Hz}, 1 \mathrm{H}), 4.62$ (t, $J=8.8 \mathrm{~Hz}, 2 \mathrm{H}), 3.86(\mathrm{~s}, 3 \mathrm{H}), 3.35$ (t, $J=8.7 \mathrm{~Hz}, 2 \mathrm{H})$; ${ }^{13} \mathrm{C}-\mathrm{NMR} \quad\left(\mathrm{CDCl}_{3}, 100 \mathrm{MHz}\right) \delta 155.5,154.0,132.6,115.0,113.9,110.5,98.7,71.5$, 56.6, 29.9; IR (film) 2959, 2229, 1603, 1481, 1464, 1437, 1420, 1319, 1238, 1074, 993, 982, $810 \mathrm{~cm}^{-1}$; HRMS-EI $(\mathrm{m} / \mathrm{z}):\left[\mathrm{M}^{+}\right]$calcd for $\mathrm{C}_{10} \mathrm{H}_{9} \mathrm{NO}_{2}: 175.0633$, found 175.0633 . 
<smiles>Cc1ccc2c(c1C#N)CCN2S</smiles>

5-Methyl-1-(toluene-4-sulfonyl)-2,3-dihydro-1H-indole-4-carbonitrile

(12):

Following the general procedure above on $0.2 \mathrm{mmol}$ of 11a, the crude product was purified by column chromatography using 30\% ether in hexanes to give $49.0 \mathrm{mg}$ (79\% Yield) and, $36.0 \mathrm{mg}$ (57\% yield) utilizing aryl bromide 11b, of $\mathbf{1 2}$ as a white solid, $\mathrm{mp}=196-197{ }^{\circ} \mathrm{C} .{ }^{1} \mathrm{H}$ NMR $\left(\mathrm{CDCl}_{3}, 400 \mathrm{MHz}\right) \delta 7.73(\mathrm{~d}, J=8.4 \mathrm{~Hz}, 1 \mathrm{H}), 7.64(\mathrm{~d}, J=8.4$ $\mathrm{Hz}, 2 \mathrm{H}), 7.25$ (d, $J=8.3 \mathrm{~Hz}, 2 \mathrm{H}), 7.13(\mathrm{~d}, J=8.5 \mathrm{~Hz}, 1 \mathrm{H}), 3.97$ (t, $J=8.5 \mathrm{~Hz}, 2 \mathrm{H}), 3.03$ (t, $J=8.5 \mathrm{~Hz}, 2 \mathrm{H}), 2.45$ (s, 3H), 2.39 (s, 3H); ${ }^{13} \mathrm{C}-\mathrm{NMR}\left(\mathrm{CDCl}_{3}, 100 \mathrm{MHz}\right) \delta 144.6,140.7$, 136.6, 136.3, 133.4, 129.8, 129.5, 127.2, 118.7, 116.0, 109.8, 49.7, 27.8, 21.5, 19.7; IR (thin film) 2956, 2224, 1597, 1472, 1357, 1168, 1092, 813, $673 \mathrm{~cm}^{-1}$; HRMS-EI (m/z): $\left[\mathrm{M}^{+}\right]$calcd for $\mathrm{C}_{17} \mathrm{H}_{16} \mathrm{~N}_{2} \mathrm{O}_{2} \mathrm{~S}: 312.0932$, found 312.0928 .<smiles>Cc1ccc2c(c1C#N)CCCN2S</smiles>

6-Methyl-1-(toluene-4-sulfonyl)-1,2,3,4-tetrahydro-quinoline-5-carbonitrile (14): Following the general procedure above on $0.2 \mathrm{mmol}$ of 13a, the crude product was purified by column chromatography using 30\% ether in hexanes to give $51.0 \mathrm{mg}$ (78 \% Yield) and, 36.0 (55\% yield) utilizing aryl bromide 13b, of 14 as a white solid, $\mathrm{mp}=172$ $173^{\circ} \mathrm{C} .{ }^{1} \mathrm{H} \mathrm{NMR}\left(\mathrm{CDCl}_{3}, 400 \mathrm{MHz}\right) \delta 7.94$ (d, $\left.J=8.6 \mathrm{~Hz}, 1 \mathrm{H}\right), 7.45(\mathrm{~d}, J=8.3 \mathrm{~Hz}$, $2 \mathrm{H}), 7.22(\mathrm{~d}, J=8.0 \mathrm{~Hz}, 2 \mathrm{H}), 7.14(\mathrm{~d}, J=8.6 \mathrm{~Hz}, 1 \mathrm{H}), 3.78-3.75 \quad(\mathrm{~m}, 2 \mathrm{H}), 2.65 \quad(\mathrm{t}, J=6.8$ $2 \mathrm{H}), 2.50(\mathrm{~s}, 3 \mathrm{H}), 2.40(\mathrm{~s}, 3 \mathrm{H}), 1.64-1.58 \quad(\mathrm{~m}, 2 \mathrm{H}) ;{ }^{13} \mathrm{C}-\mathrm{NMR} \quad\left(\mathrm{CDCl}_{3}, 100 \mathrm{MHz}\right) \delta$ $144.0,139.1,136.2$, 135.4, 133.7, 129.8, 129.3, 128.0, 127.0, 116.3, 113.1, 45.8, 25.6, 21.5, 20.3, 20.2,; IR (film) 2952, 2941, 2221, 1736, 1597, 1479, 1450, 1352, 1305, 1163, 1089, 999, 863, 814, 783, 736, 695, $665 \mathrm{~cm}^{-1}$; HRMS-EI $(\mathrm{m} / \mathrm{z}):\left[\mathrm{M}^{+}\right]$calcd for $\mathrm{C}_{18} \mathrm{H}_{18} \mathrm{~N}_{2} \mathrm{O}_{2} \mathrm{~S}: 326.1089$, found 326.1089 .<smiles>Cc1ccc2c(c1C#N)CCCCN2S</smiles>

7-Methyl-1-(toluene-4-sulfonyl)-2,3,4,5-tetrahydro-1H-benzo[b]azepine-6-

carbonitrile (16): Following the general procedure above on $0.2 \mathrm{mmol}$ of 15a, the crude product was purified by column chromatography using 30\% ether in hexanes to give 45.5 $\mathrm{mg}(67 \%$ Yield) and, 32.0mg (47\% yield) utilizing aryl bromide 15b, of $\mathbf{1 6}$ as a clear, colorless oil. ${ }^{1} \mathrm{H}$ NMR $\left(\mathrm{CDCl}_{3}, 500 \mathrm{MHz}, 45^{\circ} \mathrm{C}\right) \delta 7.63(\mathrm{~d}, J=8.0 \mathrm{~Hz}, 1 \mathrm{H}), 7.47$ (d, $J=8.1 \mathrm{~Hz}, 2 \mathrm{H}), 7.31$ (d, $J=9.6 \mathrm{~Hz}, 2 \mathrm{H}), 7.16$ (d, $J=8.1 \mathrm{~Hz}, 1 \mathrm{H}), 3.70$ (br s, 2H), 2.69 (br 
s, 2H), 2.57 (s, 3H), 2.47 (s, 3H), 1.81 (br s, 2H), 1.62 (br s, 2H); ${ }^{13} \mathrm{C}-\mathrm{NMR}\left(\mathrm{CDCl}_{3}\right.$, $75 \mathrm{MHz}) \delta 145.4,143.8,142.3,138.7,138.5,134.0,130.0,128.8,127.2,116.9,113.9$, 50.6, 31.6, 29.0, 24.7, 21.7, 21.1; IR (film)3423, 3029, 2926, 2857, 2220, 1918, 1738, 1597, 1493, 1479, 1446, 1401, 1366, 1346, 1304, 1264, 1249, 1232, 1158, 1094, 1066, 1042, 989, 949, 815802, 786, 760, 707, 685, 644, $620 \mathrm{~cm}^{-1}$; HRMS-EI (m/z): [M+ $]$ calcd forC ${ }_{19} \mathrm{H}_{20} \mathrm{~N}_{2} \mathrm{O}_{2} \mathrm{~S}: 340.1245$, found 340.1246 .<smiles>N#Cc1c2c(cc3c1CCO3)OCC2</smiles>

2,3,5,6-Tetrahydro-benzo[1,2-b;5,4-b']difuran-4-carbonitrile (18): Following the general procedure above on $0.2 \mathrm{mmol}$ of 17 , the crude product was purified by column chromatography using $10 \%$ ether in hexanes to give $30.5 \mathrm{mg}(88 \%$ Yield) of 18 as a white solid, $\mathrm{mp}=173-175{ }^{\circ} \mathrm{C} .{ }^{1} \mathrm{H}$ NMR $\left(300 \mathrm{MHz}, \mathrm{CDCl}_{3}\right) \delta 6.44(\mathrm{~s}, 1 \mathrm{H}), 4.65(\mathrm{t}, 4 \mathrm{H}, J$ $=8.8 \mathrm{~Hz}), 3.27(\mathrm{t}, 4 \mathrm{H}, J=8.8 \mathrm{~Hz}) ;{ }^{13} \mathrm{C} \mathrm{NMR}\left(75 \mathrm{MHz}, \mathrm{CDCl}_{3}\right) \delta 161.2,122.4,116.3$, 104.8, 97.5, 72.6, 29.1; IR (film) 2221, 1609, 1477, 1438, 1168, $1069 \mathrm{~cm}^{-1}$; HRMS-EI $(\mathrm{m} / \mathrm{z}):\left[\mathrm{M}^{+}\right]$calcd for $\mathrm{C}_{11} \mathrm{H}_{9} \mathrm{NO}_{2}: 187.0641$, found 187.0633 .<smiles>N#Cc1c2c(cc3c1CCCO3)OCCC2</smiles>

3,4,7,8-Tetrahydro-2H,6H-pyrano[3,2-g]chromene-5-carbonitrile (20): Following the general procedure above on $0.2 \mathrm{mmol}$ of 19, the crude product was purified by column chromatography using $10 \%$ ether in hexanes to give $32.0 \mathrm{mg}$ (75\% Yield) of 20 as a white solid, $\mathrm{mp}=149-150{ }^{\circ} \mathrm{C}$. ${ }^{1} \mathrm{H}$ NMR $\left(\mathrm{CDCl}_{3}, 400 \mathrm{MHz}\right) \delta 6.48 \quad(\mathrm{~s}, 1 \mathrm{H}), 4.16-$ $4.13(\mathrm{~m}, 4 \mathrm{H}), 2.87$ (t, $J=6.6 \mathrm{~Hz}, 4 \mathrm{H}), 2.06-1.99(\mathrm{~m}, 4 \mathrm{H}) ;{ }^{13} \mathrm{C}-\mathrm{NMR}\left(\mathrm{CDCl}_{3}, 100 \mathrm{MHz}\right) \delta$ 154.0, 118.6, 116.1, 112.9, 109.2, 66.3, 23.3, 21.7; IR (film) 2980, 2221, 1611, 1477 , 1465, 1420, 1301, 1143, $958 \mathrm{~cm}^{-1}$; HRMS-EI (m/z): [M $\left.{ }^{+}\right]$calcd for $\mathrm{C}_{13} \mathrm{H}_{13} \mathrm{NO}_{2}$ : 215.0946, found 215.0951 .<smiles>N#Cc1c2c(cc3c1CCO3)OCC2</smiles>

2,3,6,7-Tetrahydro-5H-furo[3,2-g]chromene-4-carbonitrile (22): Following the general procedure above on $0.13 \mathrm{mmol}$ of $\mathbf{2 1}$, the crude product was purified by column chromatography using $10 \%$ ether in hexanes to give $14.5 \mathrm{mg}(56 \%$ Yield) of 22 as a white solid, $\mathrm{mp}=133-135{ }^{\circ} \mathrm{C} .{ }^{1} \mathrm{H}$ NMR $\left(\mathrm{CDCl}_{3}, 400 \mathrm{MHz}\right) \delta 6.46(\mathrm{~s}, 1 \mathrm{H}), 4.61(\mathrm{t}, J=8.6$ $\mathrm{Hz}, 2 \mathrm{H}), 4.17-4.14(\mathrm{~m}, 2 \mathrm{H}), 3.29(\mathrm{t}, J=8.7 \mathrm{~Hz}, 2 \mathrm{H}), 2.86$ (t, $J=6.5 \mathrm{~Hz}, 2 \mathrm{H}), 2.05-1.99$ $(\mathrm{m}, 2 \mathrm{H}) ;{ }^{13} \mathrm{C}-\mathrm{NMR}\left(\mathrm{CDCl}_{3}, 100 \mathrm{MHz}\right) \delta 159.3,155.3,124.0,116.6,116.2,108.7,103.0$, 
71.8, 66.4, 29.0, 23.3, 21.6; IR (film) 2979, 2223, 1734, 1615, 1474, 1460, 1442, 1395, 1361, 1339, 1320, 1230, 1192, 1167, 1118, 1082, 982, 945, $855 \mathrm{~cm}^{-1}$; HRMS-EI (m/z): $\left[\mathrm{M}^{+}\right]$calcd for $\mathrm{C}_{12} \mathrm{H}_{11} \mathrm{NO}_{2}: 201.0789$, found 201.0789.

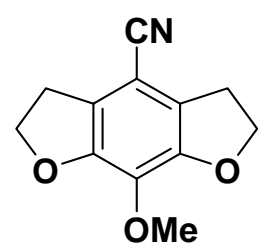

8-Methoxy-2,3,5,6-tetrahydro-benzo[1,2-b;5,4-b']difuran-4-carbonitrile

(24):

Following the general procedure above on $0.2 \mathrm{mmol}$ of $\mathbf{2 3}$, the crude product was purified by column chromatography using $10 \%$ ether in hexanes to give $32.0 \mathrm{mg}$ (74 \% Yield) of 24 as a white solid, $\mathrm{mp}=160-161{ }^{\circ} \mathrm{C} .{ }^{1} \mathrm{H}$ NMR $\left(\mathrm{CDCl}_{3}, 400 \mathrm{MHz}\right) \delta 4.68(\mathrm{t}$, $J=8.8 \mathrm{~Hz}, 4 \mathrm{H}), 4.05(\mathrm{~s}, 3 \mathrm{H}), 3.29(\mathrm{t}, J=8.8 \mathrm{~Hz}, 4 \mathrm{H}) ;{ }^{13} \mathrm{C}-\mathrm{NMR} \quad\left(\mathrm{CDCl}_{3}, 100 \mathrm{MHz}\right) \delta$ 150.4, 133.8, 124.7, 116.3, 97.2, 72.8, 60.2, 29.4; IR (film) 2964, 2218, 1734, 1718, $1610,1584,1502,1473,1458,1447,1424,1387,1345,1263,1192,1131,1064,1015$, 9999, 982, 895, 878, 747, $702 \mathrm{~cm}^{-1}$; HRMS-ESI $(\mathrm{m} / \mathrm{z}):\left[\mathrm{M}^{+}\right]$calcd for $\left[\mathrm{C}_{12} \mathrm{H}_{12} \mathrm{NO}_{3}\right]^{+}$: 218.0811, found 218.0819. 


\section{Modification of Benzonitrile Products}<smiles>NCc1c2c(cc3c1CCO3)OCC2</smiles>

2,3,5,6-Tetrahydro-benzo[1,2-b;5,4-b']difuran-4-ylmethyl-ammonium; chloride (25): To a solution of $18(56.1 \mathrm{mg}, 0.3 \mathrm{mmol})$ in $6 \mathrm{ml}$ anhydrous THF at $0{ }^{\circ} \mathrm{C}$ under nitrogen was added $\mathrm{LiAlH}_{4}(34.2 \mathrm{mg}, 0.3 \mathrm{mmol})$. The reaction was warmed to room temperature and stirred for 6 hours. Solid sodium potassium tartrate $(100 \mathrm{mg})$ was added followed by $5 \mathrm{ml}$ of a saturated sodium potassium tartrate solution and stirring continued for 1 hour. The resulting solution was extracted with ether. The organic phase was washed with water and brine and then dried over magnesium sulfate. The clear solution was then concentrated under reduced pressure and the redisolved in anhydrous ether. To this clear solution was added a $1 \mathrm{M}$ solution of hydrogen chloride in ether $(1 \mathrm{ml})$. The precipitate formed was filter and washed with anhydrous ether to give $64 \mathrm{mg}$ (94\% yield) of 25 as a pale yellow solid, decomposition $230{ }^{\circ} \mathrm{C} .{ }^{1} \mathrm{H}$ NMR $\left(\mathrm{D}_{2} \mathrm{O}, 300 \mathrm{MHz}\right) \delta 6.38$ $(\mathrm{s}, 1 \mathrm{H}), 4.65$ (t, $J=8.6 \mathrm{~Hz}, 4 \mathrm{H}), 4.11$ (br s, $2 \mathrm{H}), 3.20(\mathrm{t}, J=8.6 \mathrm{~Hz}, 4 \mathrm{H}) ;{ }^{13} \mathrm{C}-\mathrm{NMR}\left(\mathrm{D}_{2} \mathrm{O}\right.$, $75 \mathrm{MHz}) \delta 159.7,125.1,119.5,93.4,72.7,38.7,27.1$; IR (film) 2908, 2366, 2096, $1605,1458,1448,1387,1319,1257,1213,1175,1062,981,938,833 \mathrm{~cm}^{-1}$; HRMS-EI $(\mathrm{m} / \mathrm{z}):\left[\mathrm{M}^{+}\right]$calcd for $\left[\mathrm{C}_{11} \mathrm{H}_{14} \mathrm{NO}_{2}\right]^{+}:$191.0946, found 196.0949 .<smiles>NC(=O)c1c2c(cc3c1CCO3)OCC2</smiles>

2,3,5,6-Tetrahydro-benzo[1,2-b;5,4-b']difuran-4-carboxylic acid amide (26): To a solution of 18 (37.4 mg, $0.2 \mathrm{mmol})$ in $95 \%$ ethanol was added a $5 \mathrm{M}$ solution of sodium hydroxide (16 uL, $0.12 \mathrm{mmol})$ and hydrogen peroxide $(100 \mathrm{uL}, 1.5 \mathrm{mmol})$ and stirred for 8 hours at $60{ }^{\circ} \mathrm{C}$. The resulting solution was concentrated under reduced pressure to give a off white solid which was washed with water and dried under reduced pressure to give $31.5 \mathrm{mg}$ (77\% yield) of 26, decomposition $217^{\circ} \mathrm{C} .{ }^{1} \mathrm{H}$ NMR (DMSO, $\left.400 \mathrm{MHz}\right) \delta$ 7.46 (br s, $1 \mathrm{H}), 7.42$ (br s, $1 \mathrm{H}), 6.30$ (s, $1 \mathrm{H}), 4.50$ (t, $J=8.7 \mathrm{~Hz}, 4 \mathrm{H}), 3.16$ (t, $J=8.7 \mathrm{~Hz}$, $4 \mathrm{H}) ;{ }^{13} \mathrm{C}-\mathrm{NMR}$ (DMSO, $\left.100 \mathrm{MHz}\right) \delta 168.4,159.9,129.8,116.3,93.1,71.6,28.4$; IR (film) 3361, 2958, 2934, 2254, 2127, 1653, 1587, 1472, 1450, 1412, 1368, 1312, 1263 , 1156, 1124, 1083, 1025, 1008, 951, 824, $763 \mathrm{~cm}^{-1}$; HRMS-EI (m/z): $\left[\mathrm{M}^{+}\right]$calcd for $\mathrm{C}_{11} \mathrm{H}_{12} \mathrm{NO}_{3}$ : 206.0811, found 206.0814. 


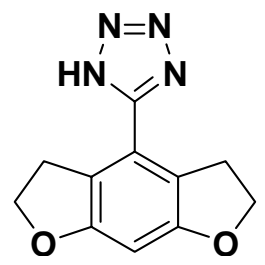

5-(2,3,5,6-Tetrahydro-benzo[1,2-b;5,4-b']difuran-4-yl)-1H-tetrazole $\quad$ (27): $\quad$ To a microwave vial with magnetic stir bar was added 18 (37.4 $\mathrm{mg} 0.2 \mathrm{mmol}$ ) was added sodium azide (156 mg, 2.4mmol) and ammonium chloride (128mg, $2.4 \mathrm{mmol})$. The vial was sealed and $2 \mathrm{ml}$ of anhydrous DMF was added. The vial was purged with nitrogen for 5 minutes and irradiated for 25 minutes at $220{ }^{\circ} \mathrm{C}$. The resulting solution was extracted with chloroform, washed with water and brine and concentrated under reduced pressure. The crude product was purified by preparatory TLC using ethyl acetate to give $16.0 \mathrm{mg}$ (35\% yield) of 27 as a off-white solid, $\mathrm{mp}=114-115^{\circ} \mathrm{C} .{ }^{1} \mathrm{H}$ NMR $\left(\mathrm{CDCl}_{3}, 400\right.$ $\mathrm{MHz}) \delta 6.45 \quad(\mathrm{~s}, 1 \mathrm{H}), 4.67 \quad(\mathrm{t}, J=8.7 \mathrm{~Hz}, 4 \mathrm{H}), 3.50(\mathrm{t}, J=8.7 \mathrm{~Hz}, 4 \mathrm{H}), 1.26 \quad(\mathrm{~s}, 1 \mathrm{H})$ $\left(\mathrm{CDCl}_{3}, 100 \mathrm{MHz}\right) \delta 161.3,117.8,94.9,77.2,72.3,30.5,29.7$; IR (film) 3449, 2981, 2972, 2252, 1792, 1700, 1609, 1545, 1482, 1458, 1382, 1317, 1297, 1263, 1163, 1095, $1069,1042,990,729,685 \mathrm{~cm}-1$; HRMS-EI $(\mathrm{m} / \mathrm{z}):\left[\mathrm{M}^{+}\right]$calcd for $\left[\mathrm{C}_{11} \mathrm{H}_{11} \mathrm{~N}_{4} \mathrm{O}_{2}\right]^{+}$: 231.0876 , found 231.0874 . 
Spectral Data

(In numerical order) 
4- (2-Bromo-ethoxy)-2-iodo-1-methyl-benzene (1)

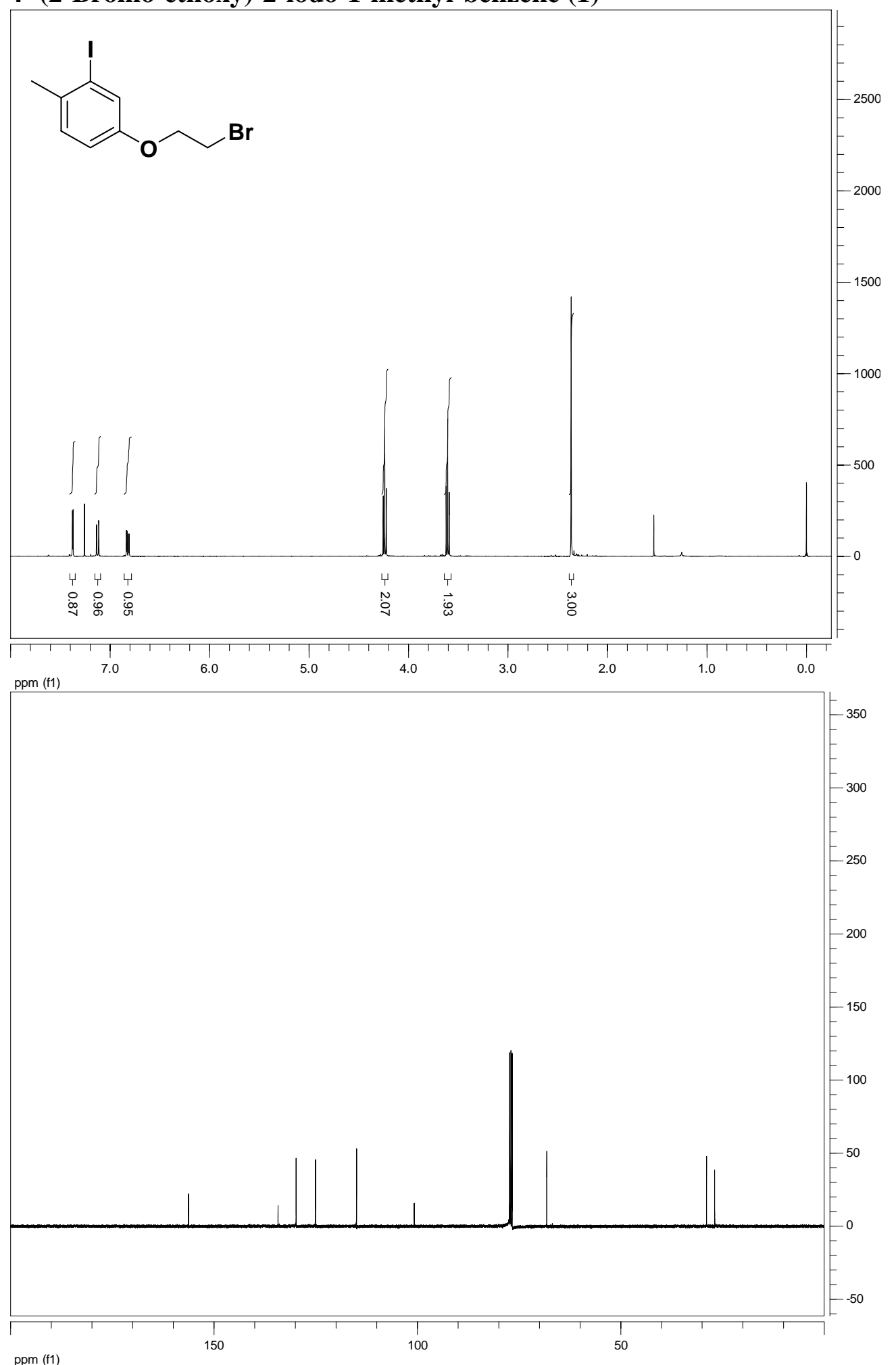

ppm (f1) 
5-Methyl-2,3-dihydro-benzofuran-4-carbonitrile (2)
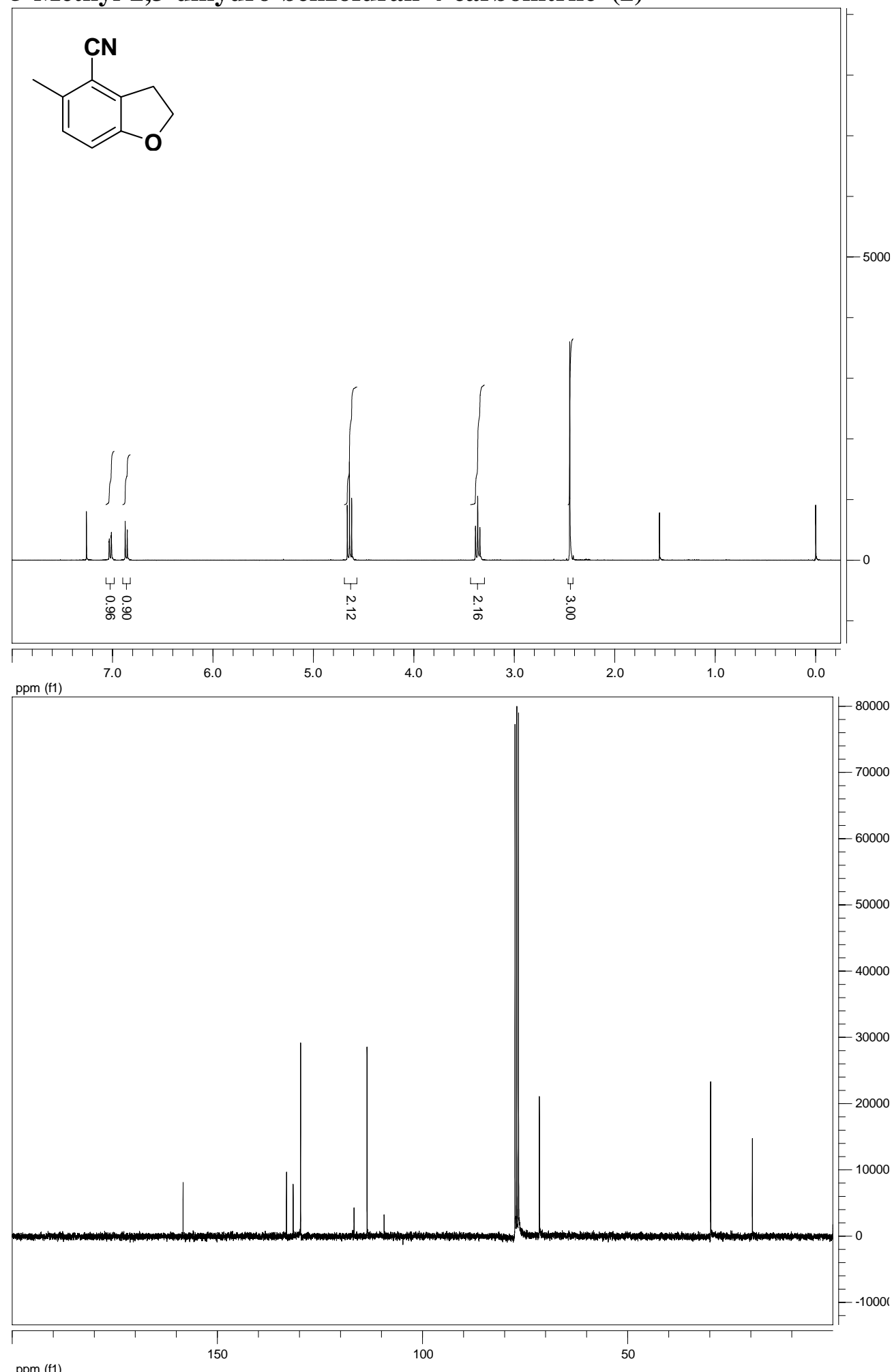

ppm (f1) 

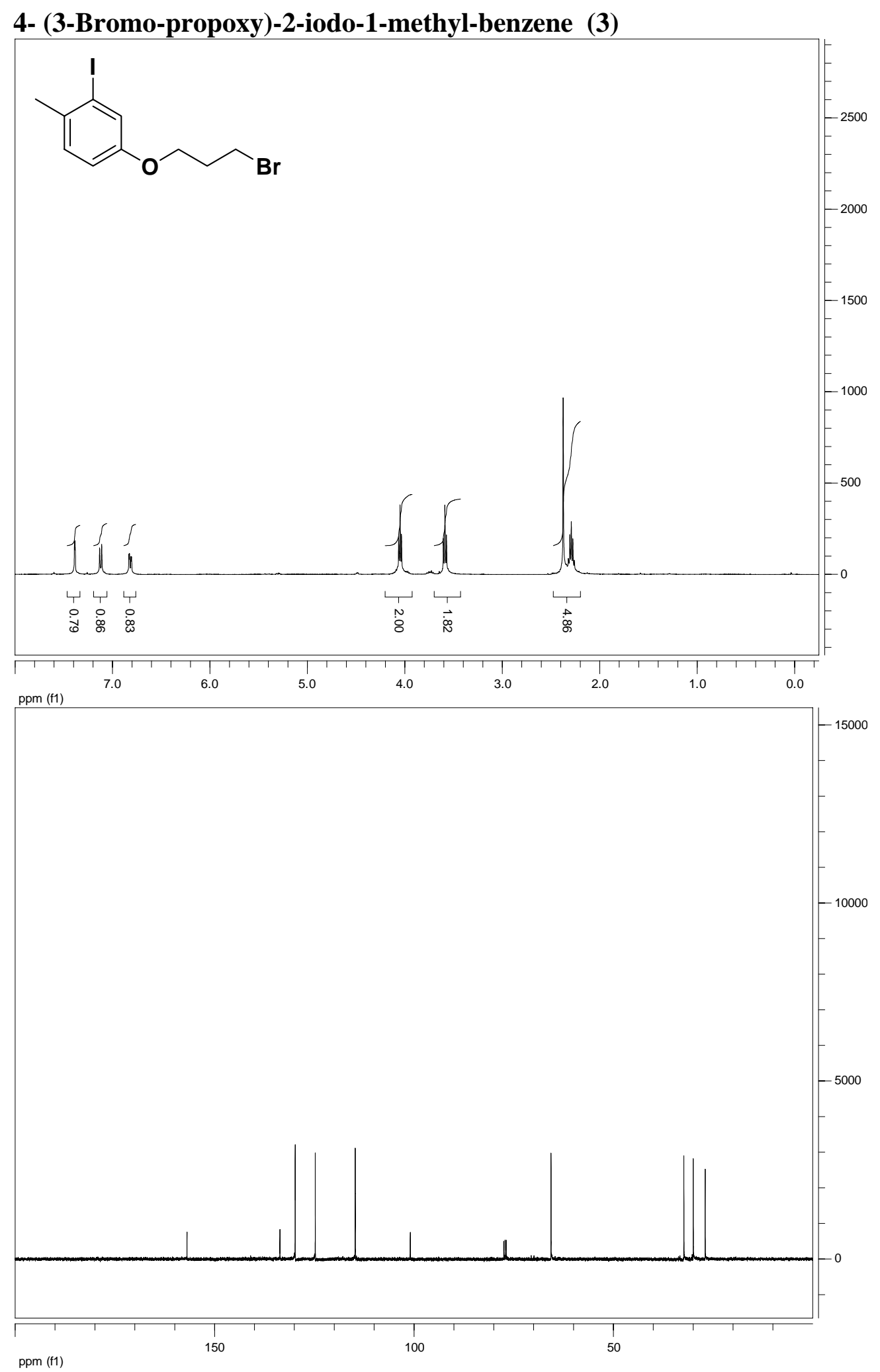

ppm (f1) 
6-Methyl-chroman-5-carbonitrile (4)

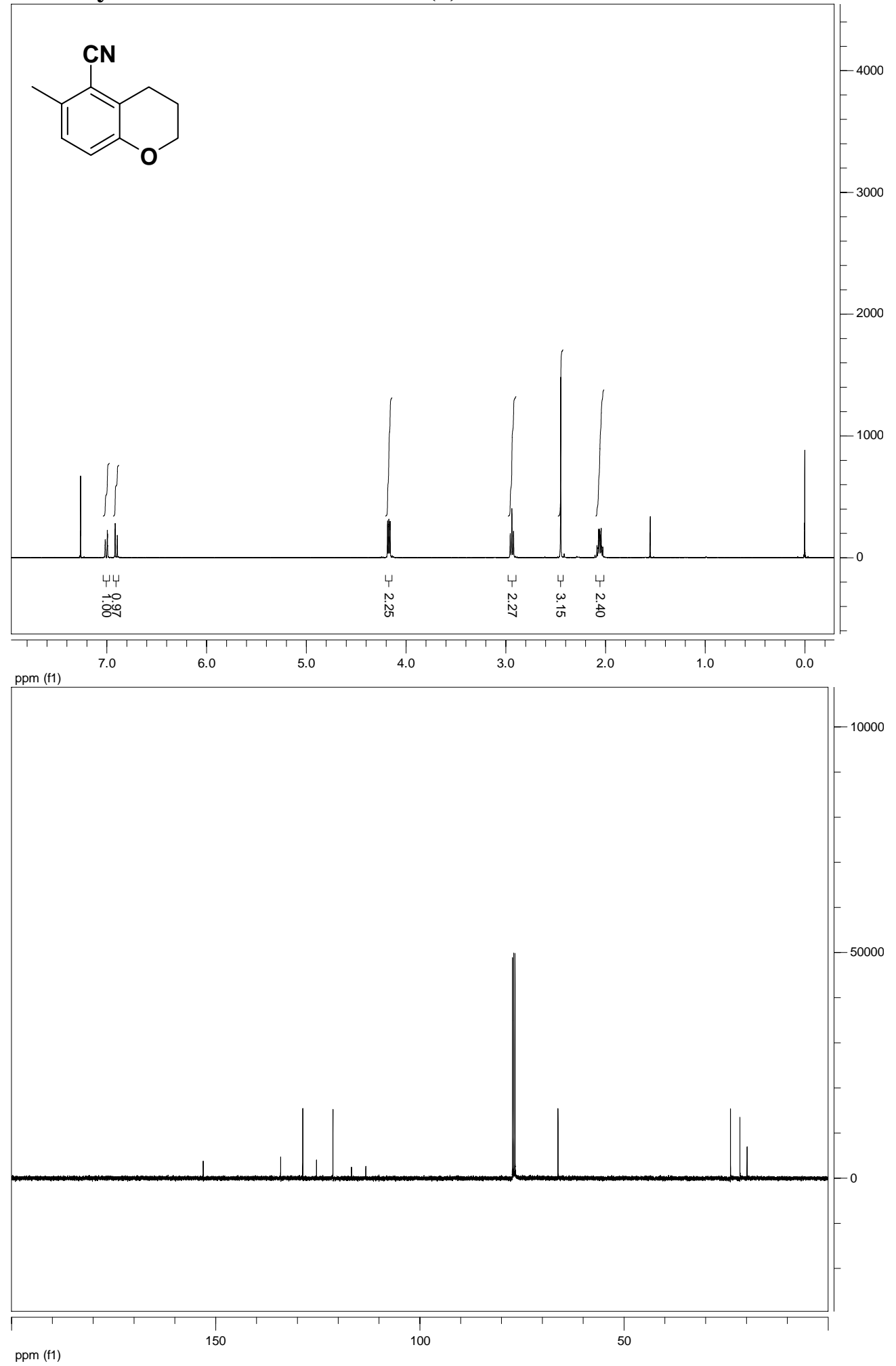


4- (4-Bromo-butoxy)-2-iodo-1-methyl-benzene (5)
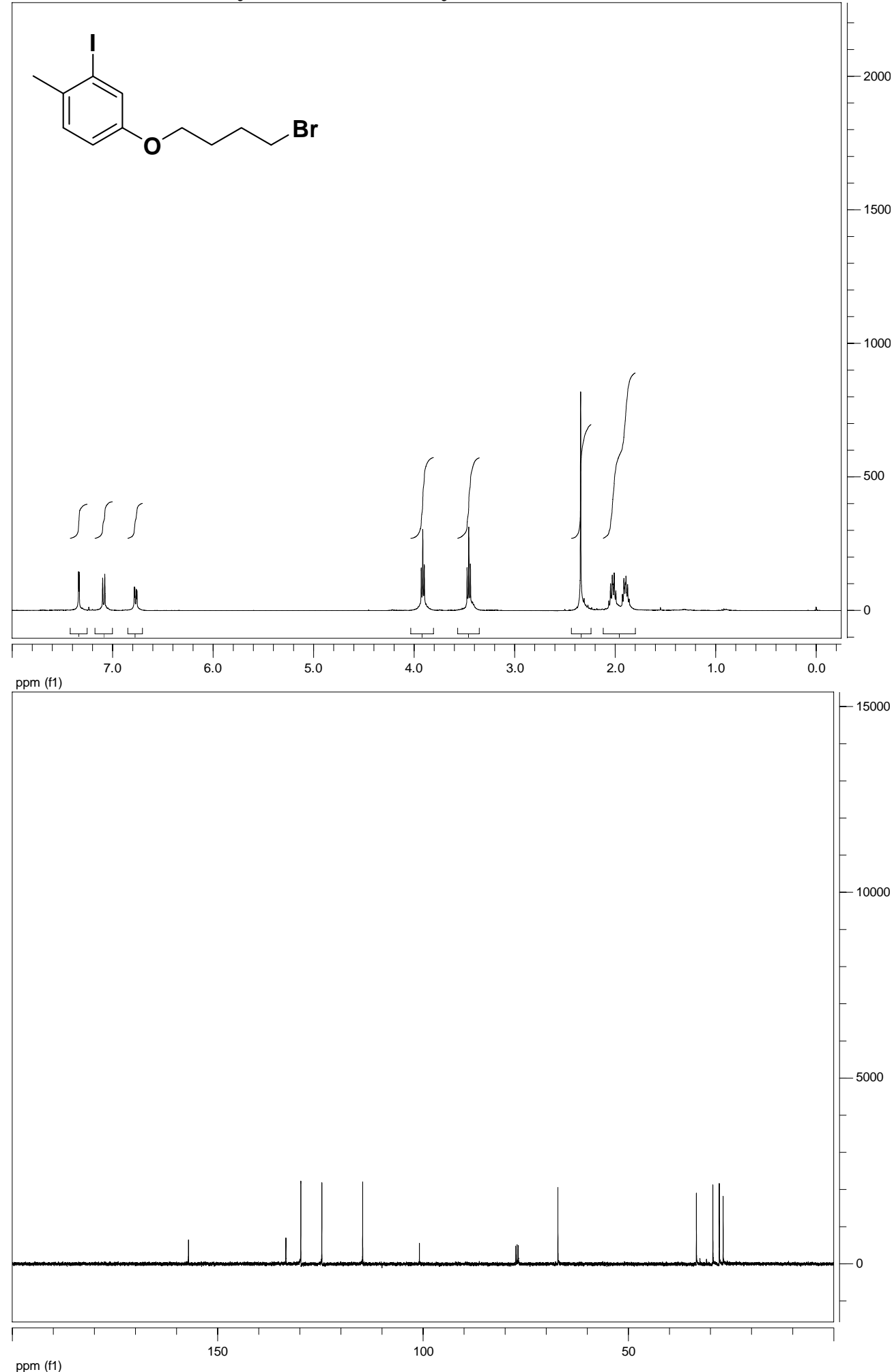
7-Methyl-2,3,4,5-tetrahydro-benzo[b]oxepine-6-carbonitrile (6)
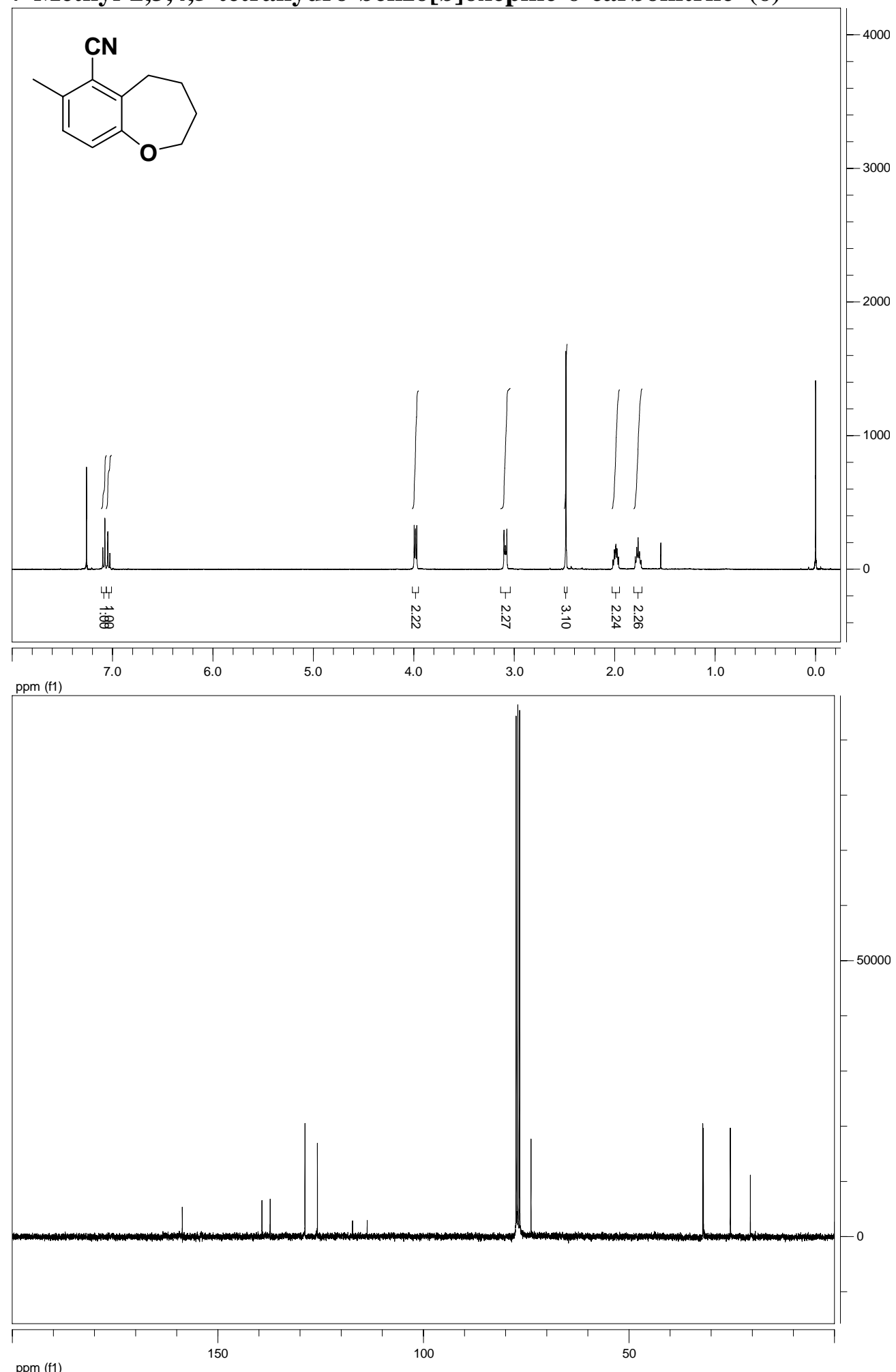
4- (2-Bromo-ethoxy)-2-iodo-1-nitro-benzene (7)
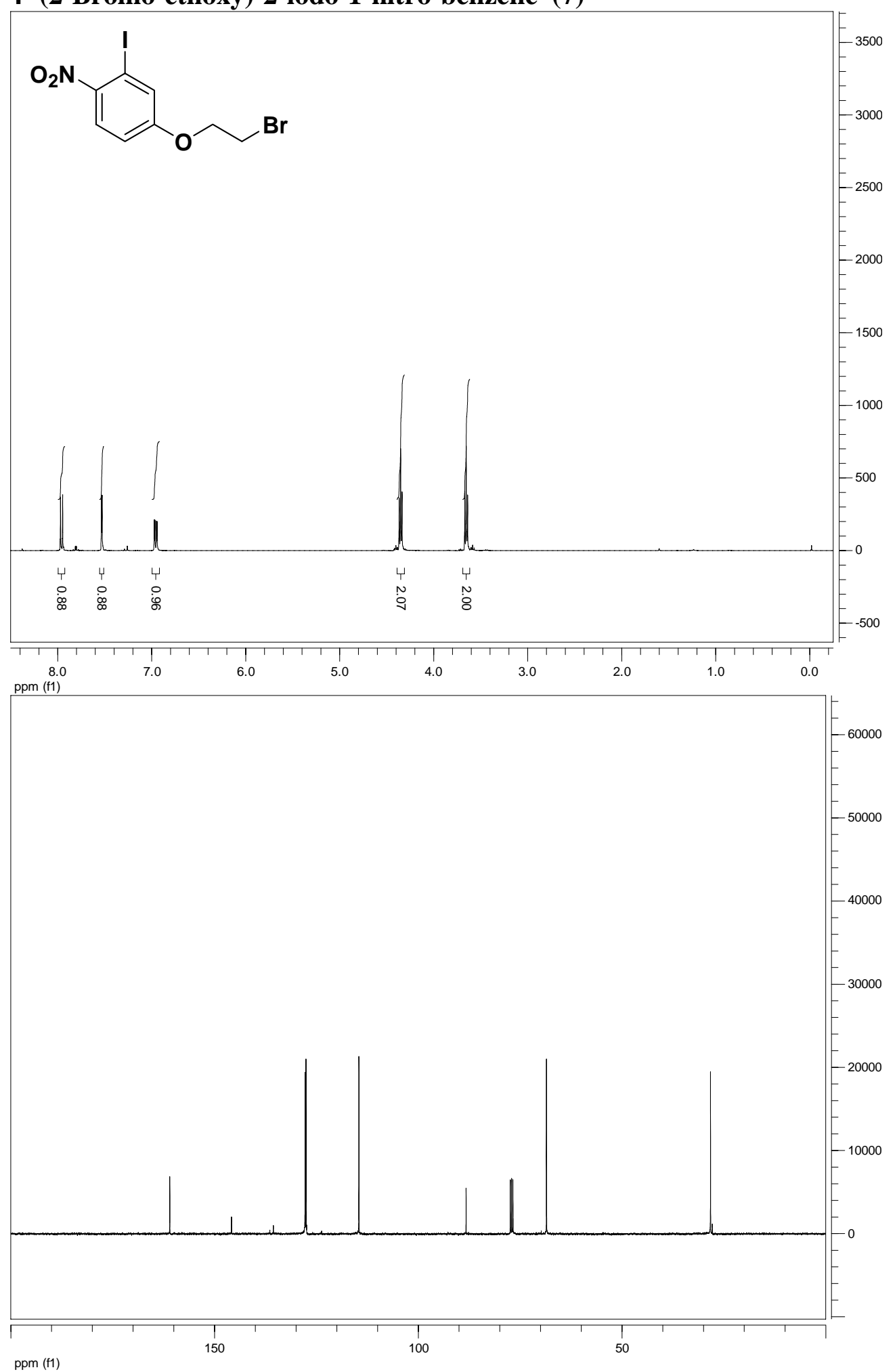
5-Methoxy-2,3-dihydro-benzofuran-4-carbonitrile (8)
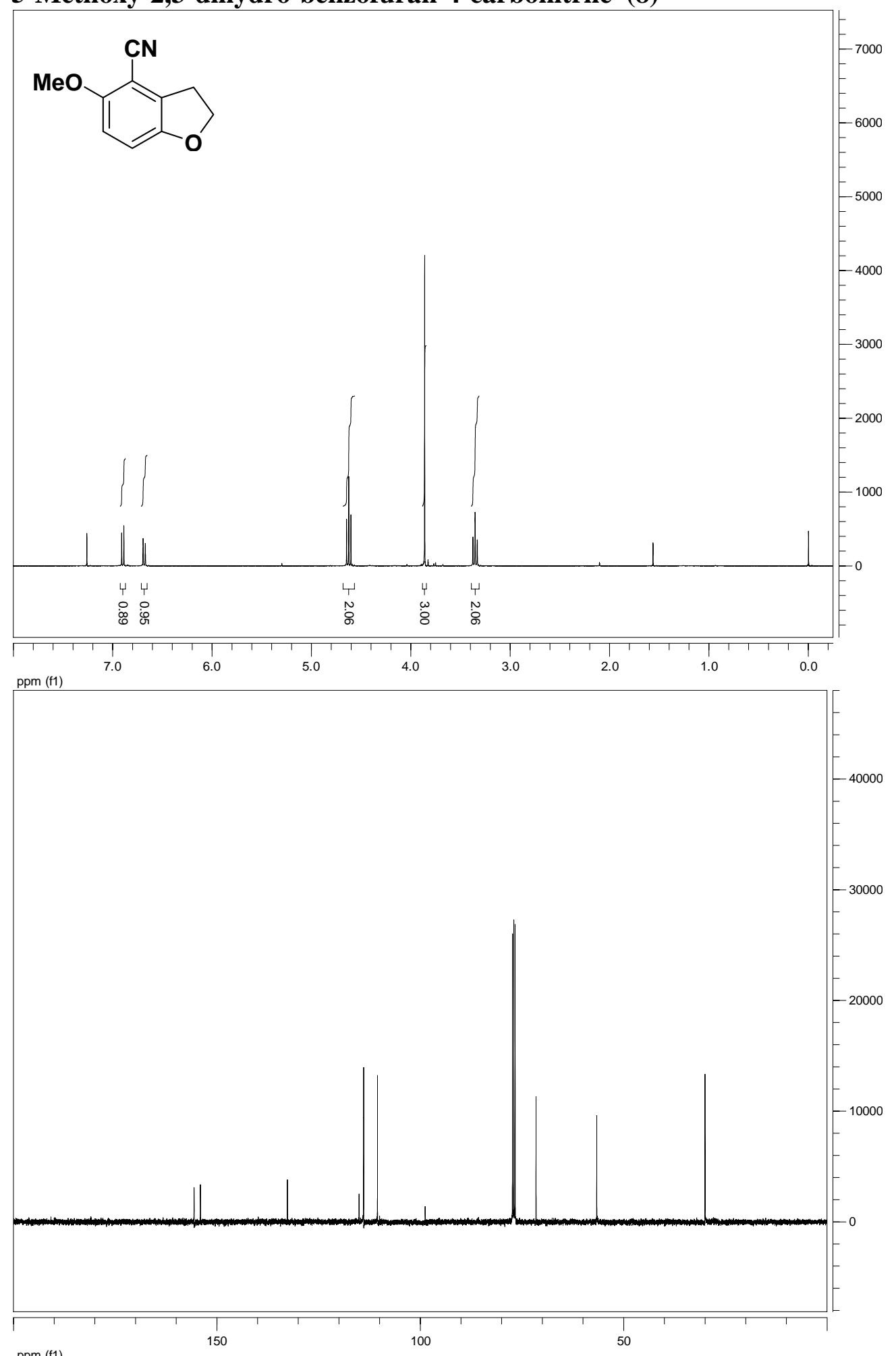

ppm (f1) 
4- (2-Bromo-ethoxy)-2-iodo-1-methoxy-benzene (9)
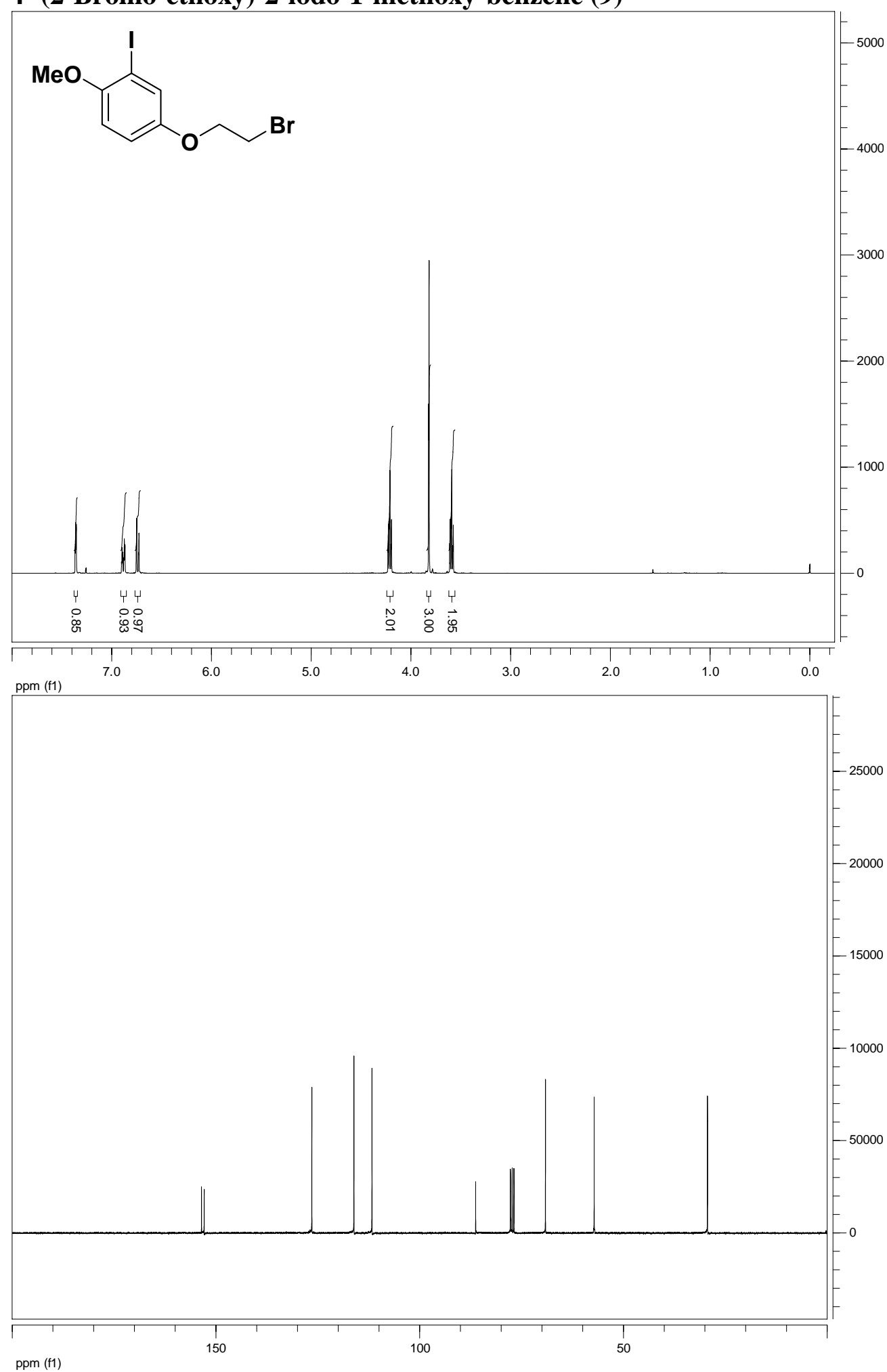
5-Nitro-2,3-dihydro-benzofuran-4-carbonitrile (10)

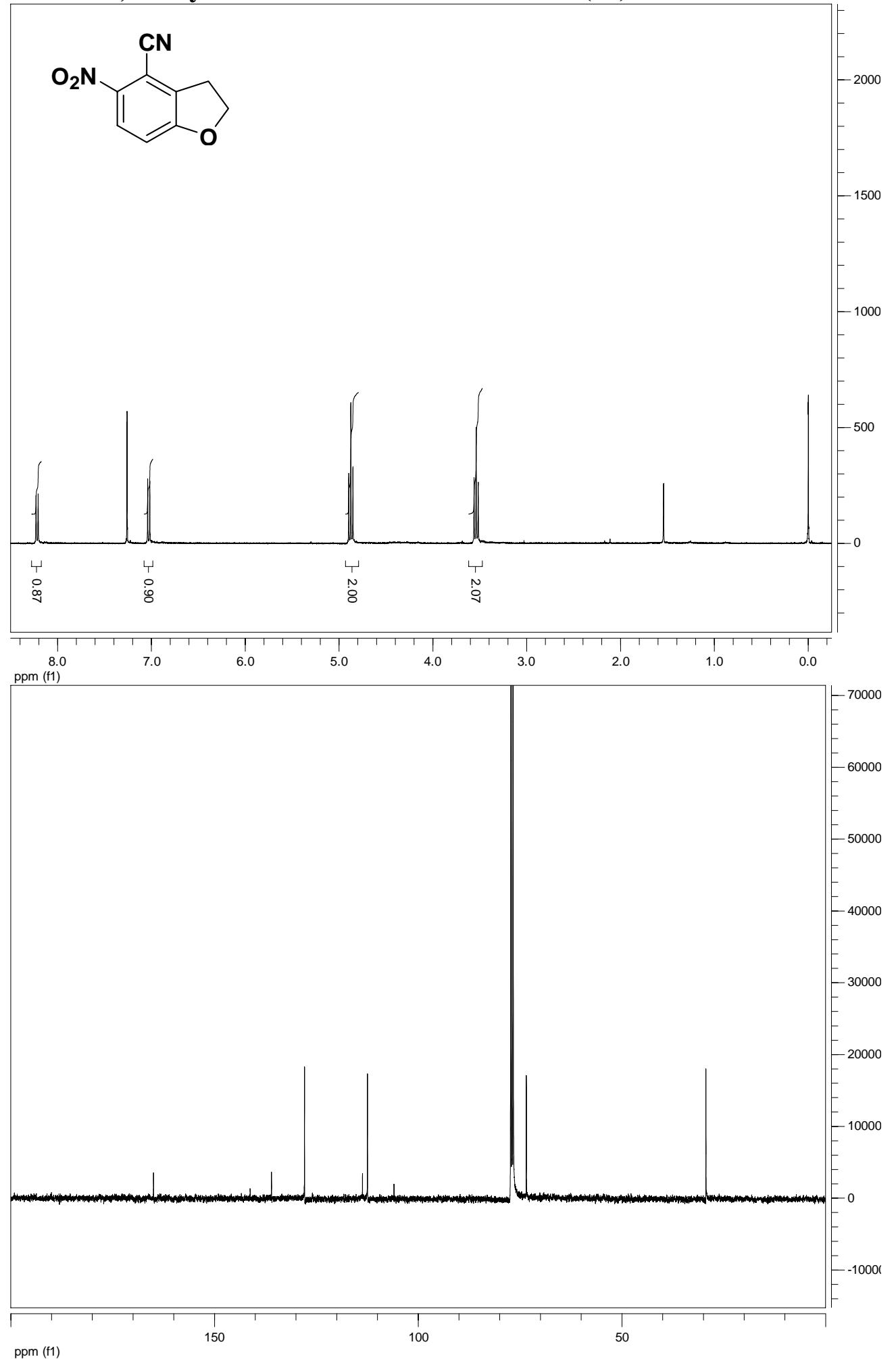


$N$ - (2-Bromo-ethyl)- $N$ - (3-iodo-4-methyl-phenyl)-4-methyl-benzenesulfonamide (11a)
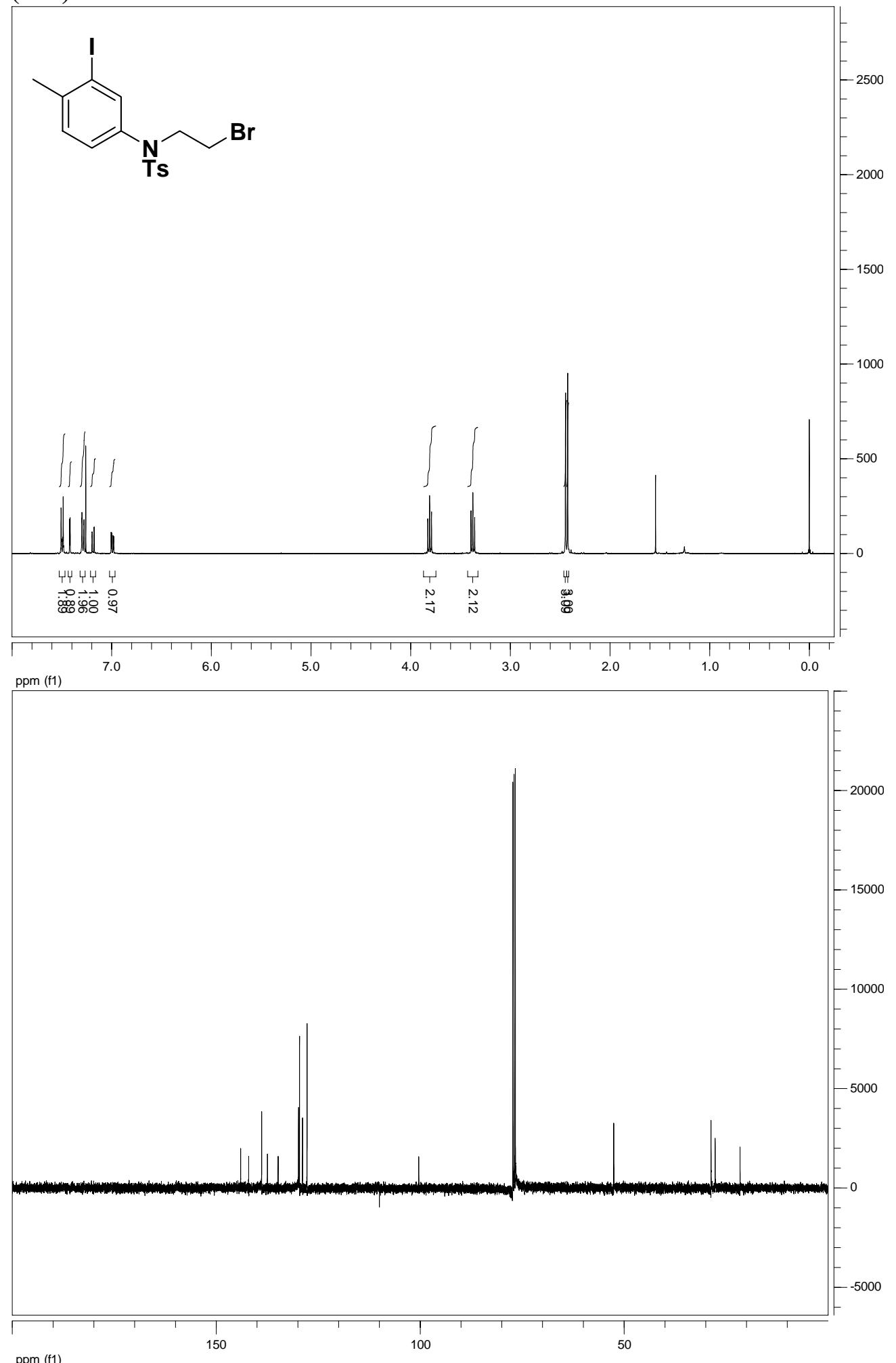

ppm (f1) 
$N$-(2-Bromo-ethyl)- $N$-(3-bromo-4-methyl-phenyl)-4-methyl-benzenesulfonamide (11b)
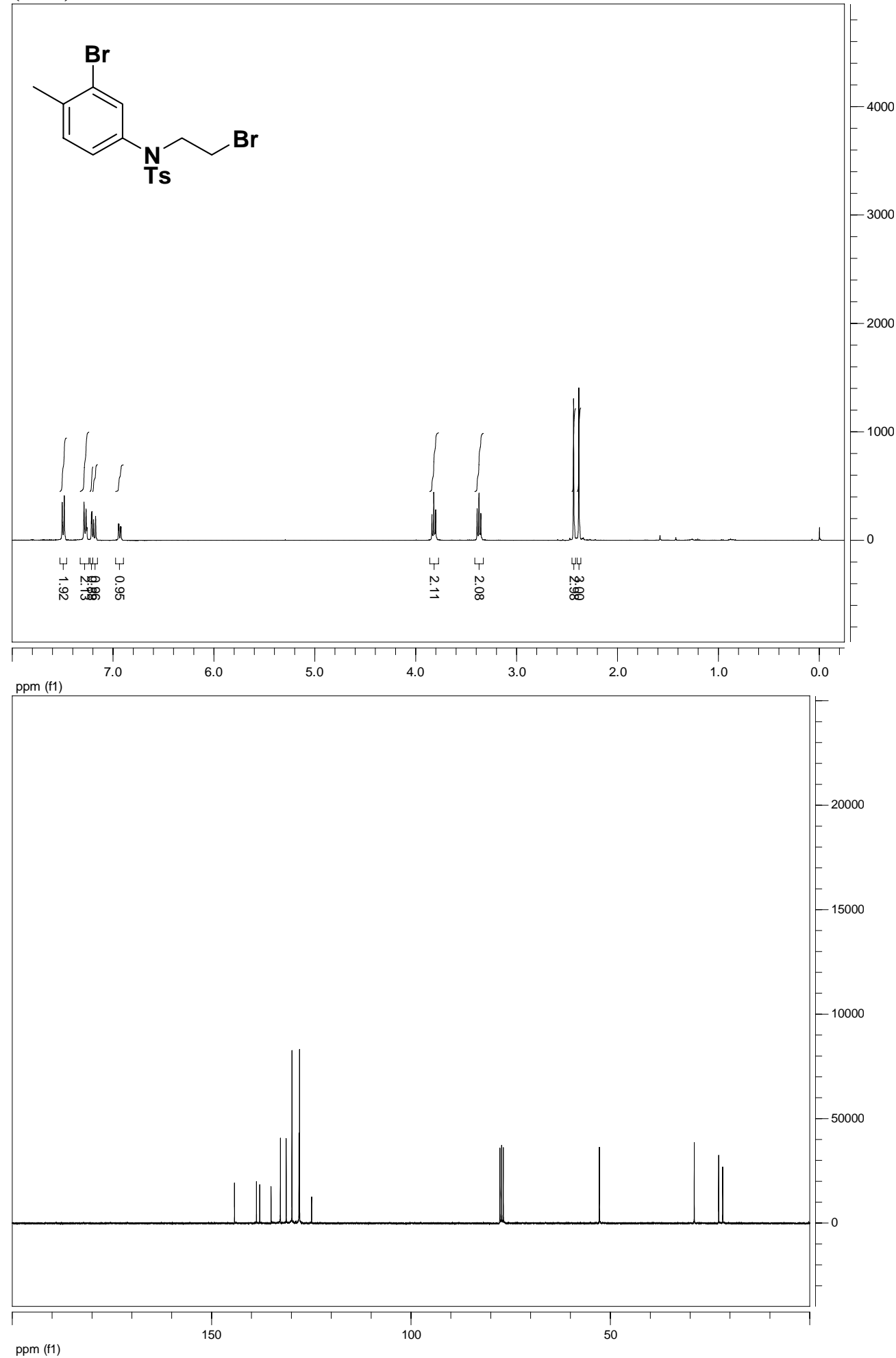
5-Methyl-1-(toluene-4-sulfonyl)-2,3-dihydro-1H-indole-4-carbonitrile (12)
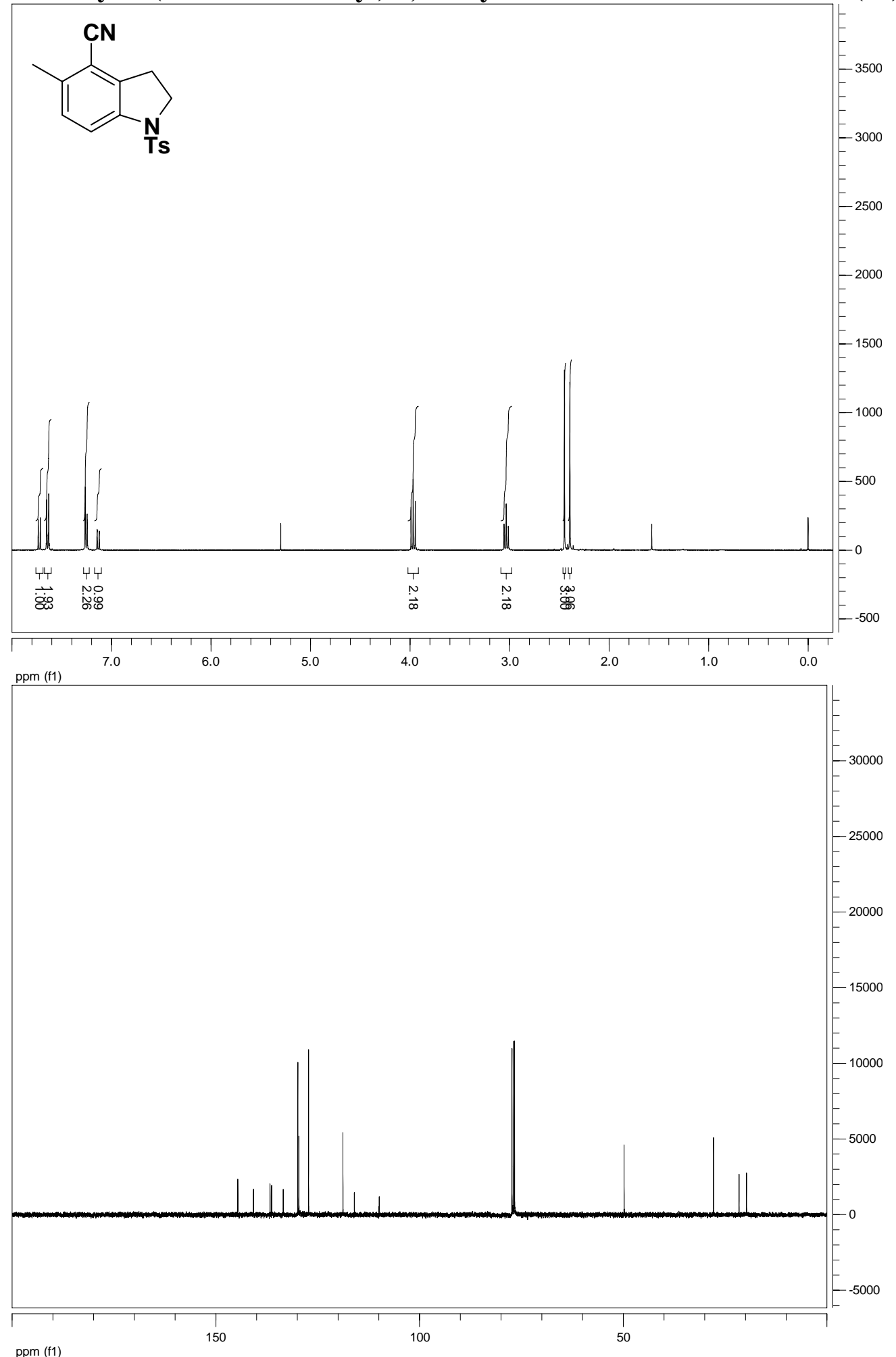
$N$-(3-Bromo-propyl)- $N$-(3-iodo-4-methyl-phenyl)-4-methyl-benzenesulfonamide (13a)
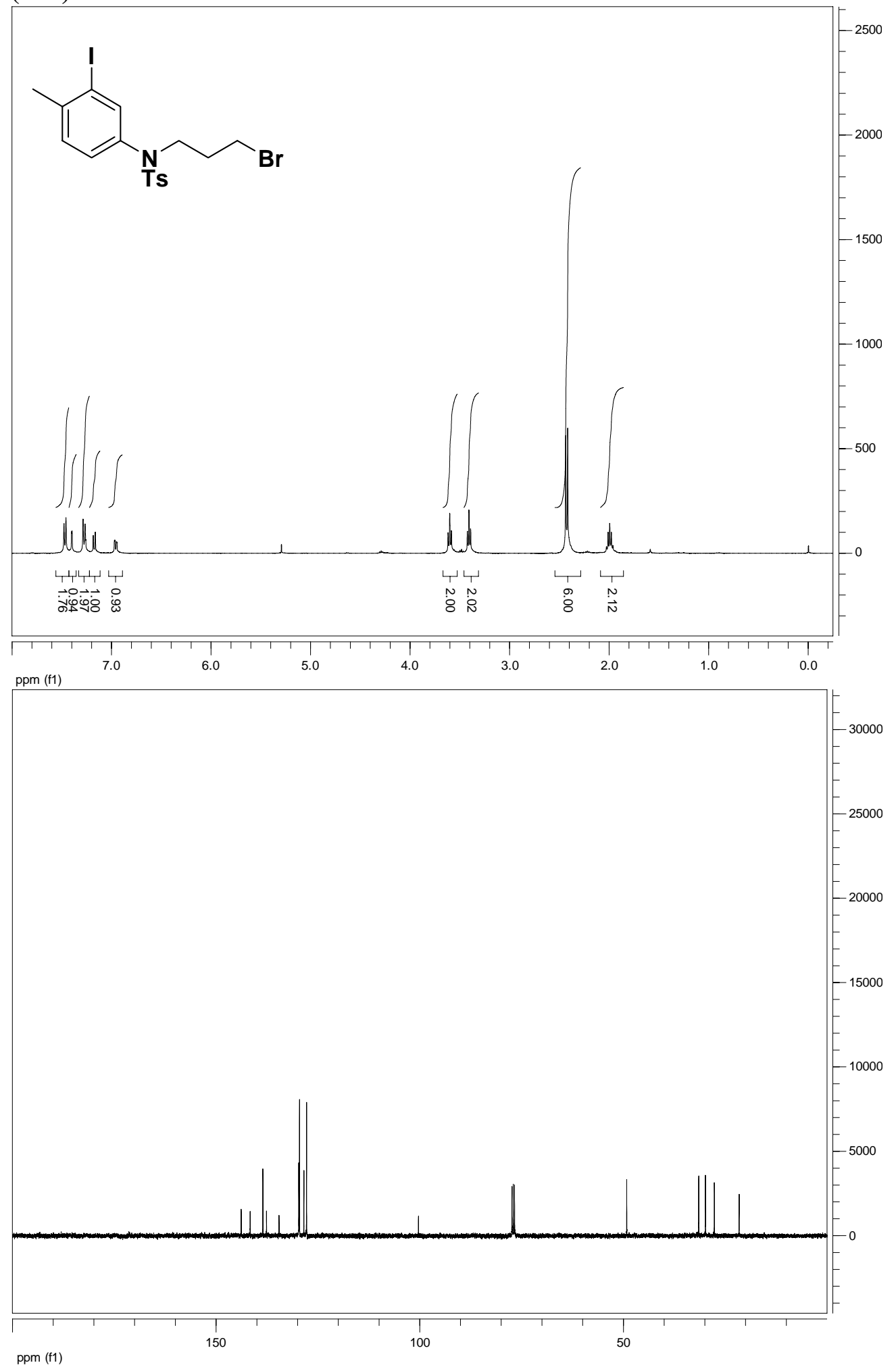
$N$-(3-Bromo-4-methyl-phenyl)- $N$-(3-bromo-propyl)-4-methyl-benzenesulfonamide (13b)
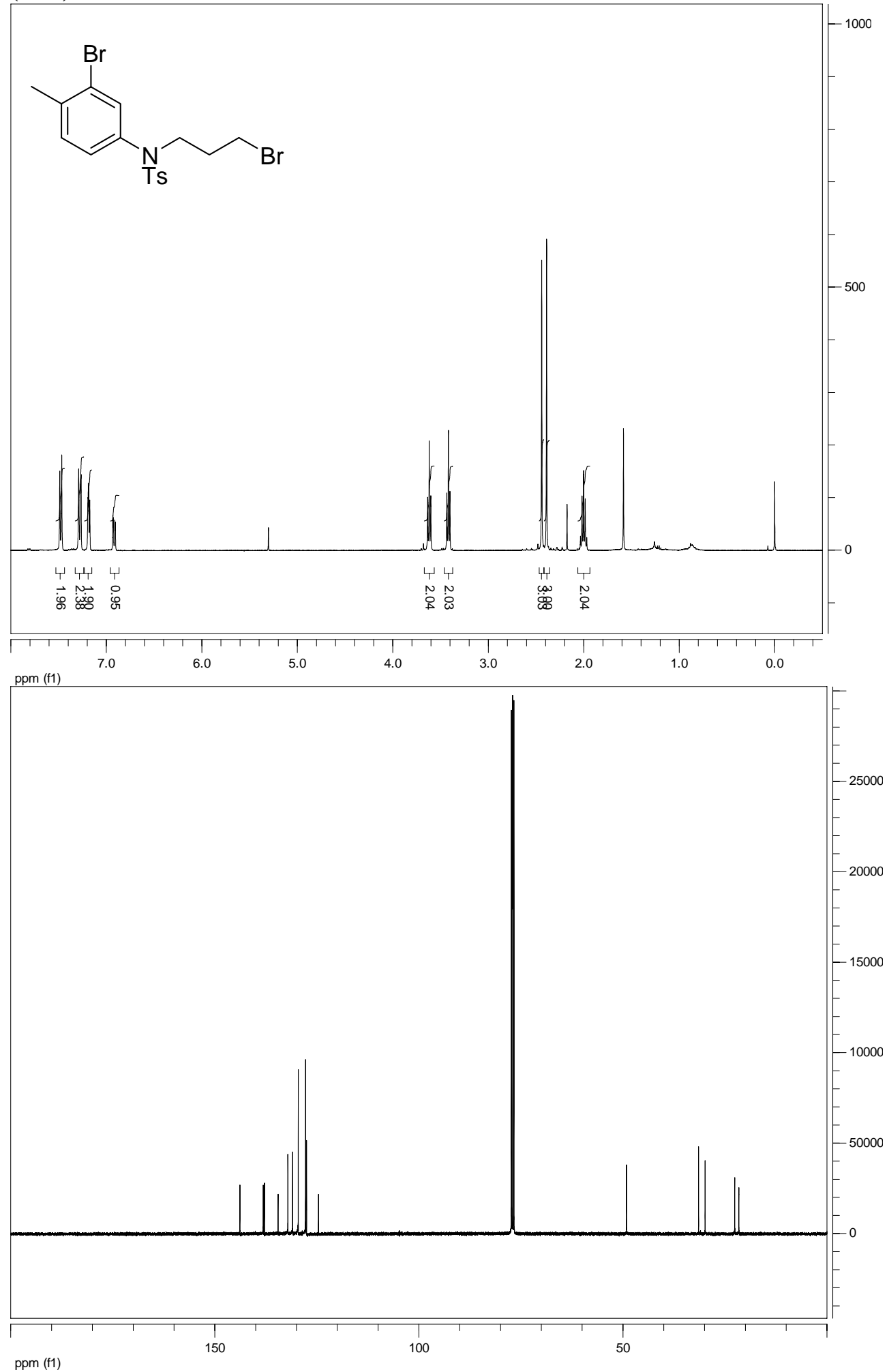
6-Methyl-1-(toluene-4-sulfonyl)-1,2,3,4-tetrahydro-quinoline-5-carbonitrile (14)

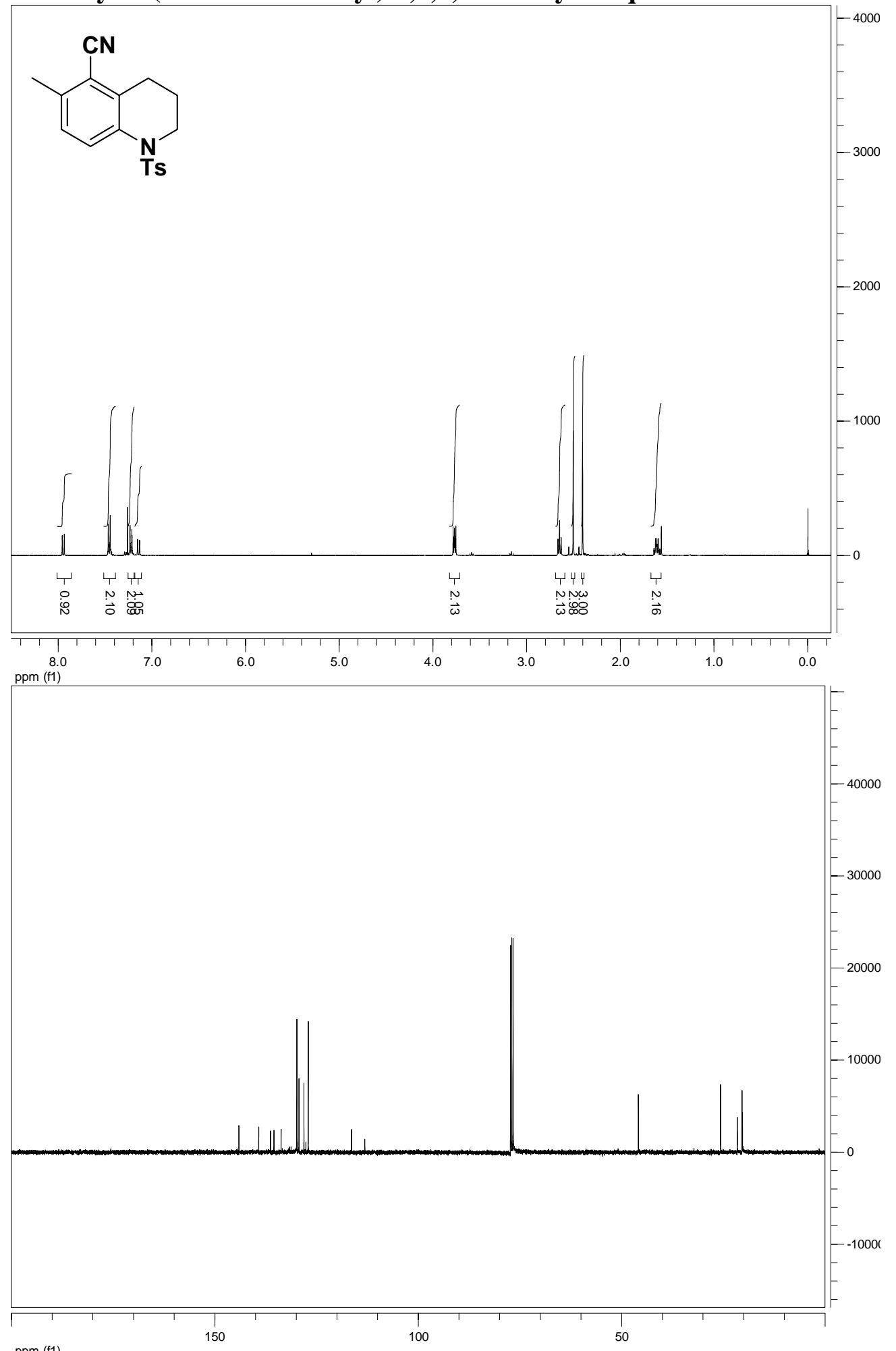

ppm (f1) 
$N$-(4-Bromo-butyl)- $N$-(3-iodo-4-methyl-phenyl)-4-methyl-benzenesulfonamide (15a)

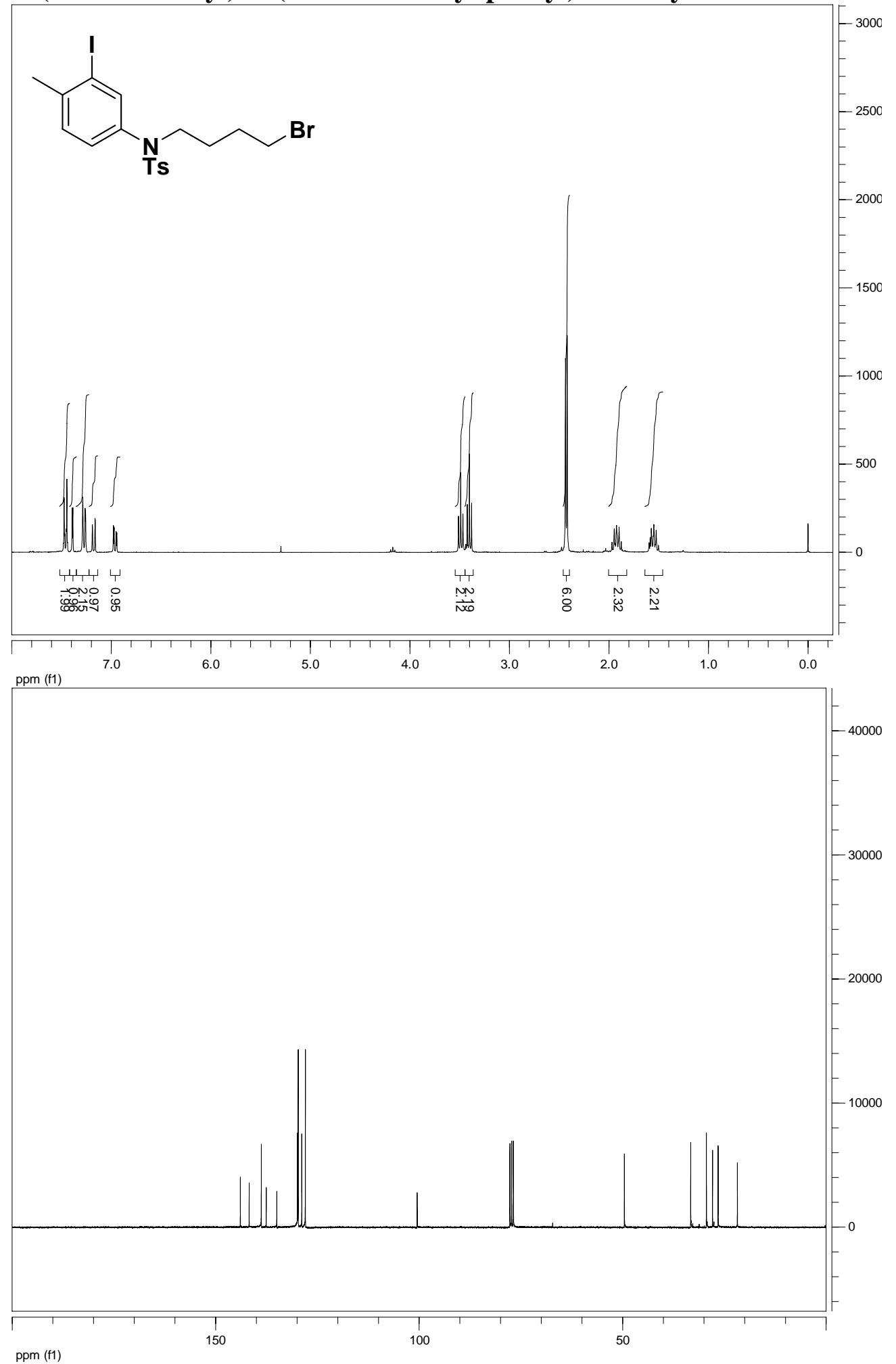


$N$-(4-Bromo-butyl)- $N$-(3-bromo-4-methyl-phenyl)-4-methyl-benzenesulfonamide (15b)
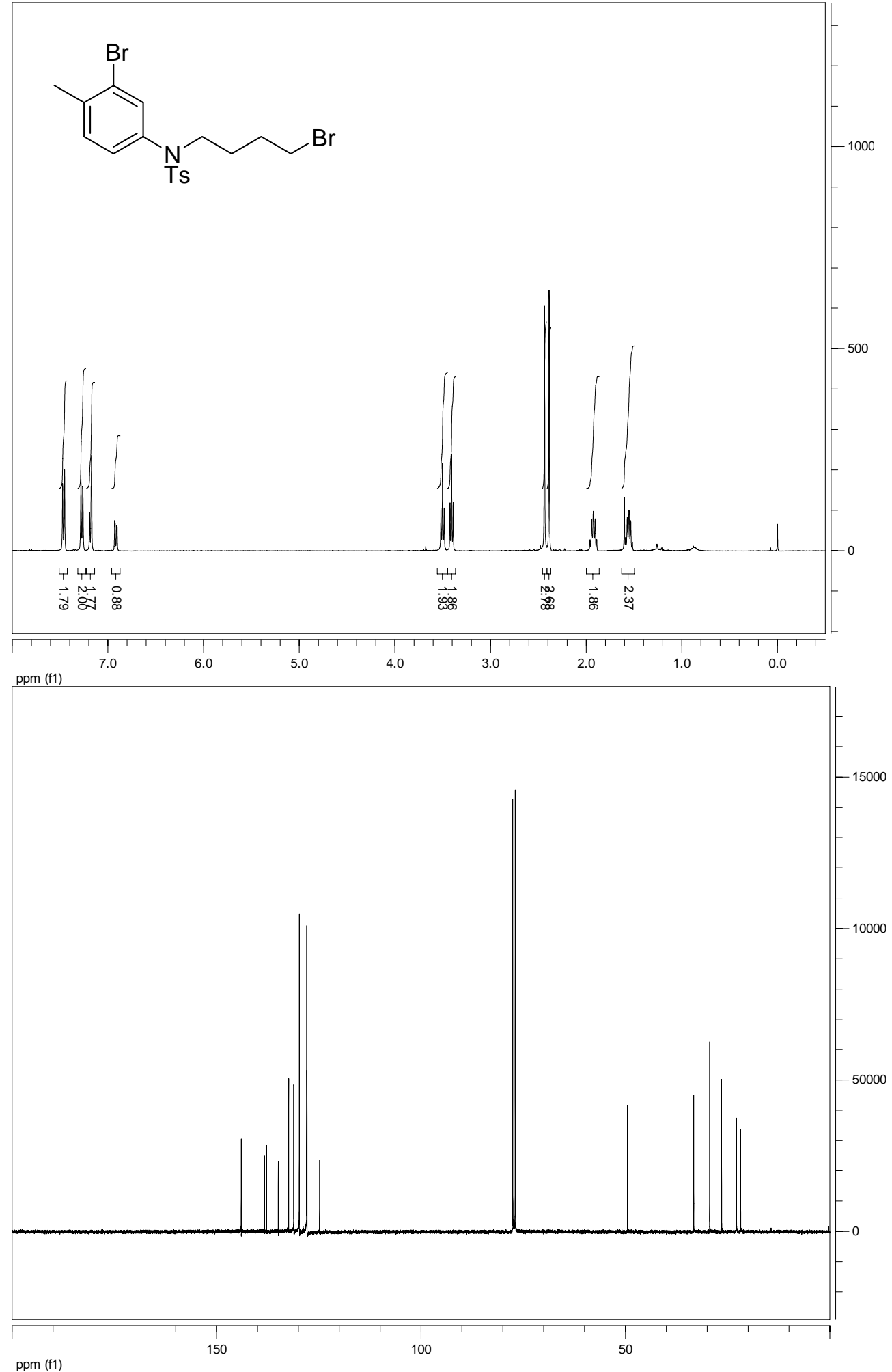
7-Methyl-1-(toluene-4-sulfonyl)-2,3,4,5-tetrahydro-1H-benzo[b]azepine-6carbonitrile (16)
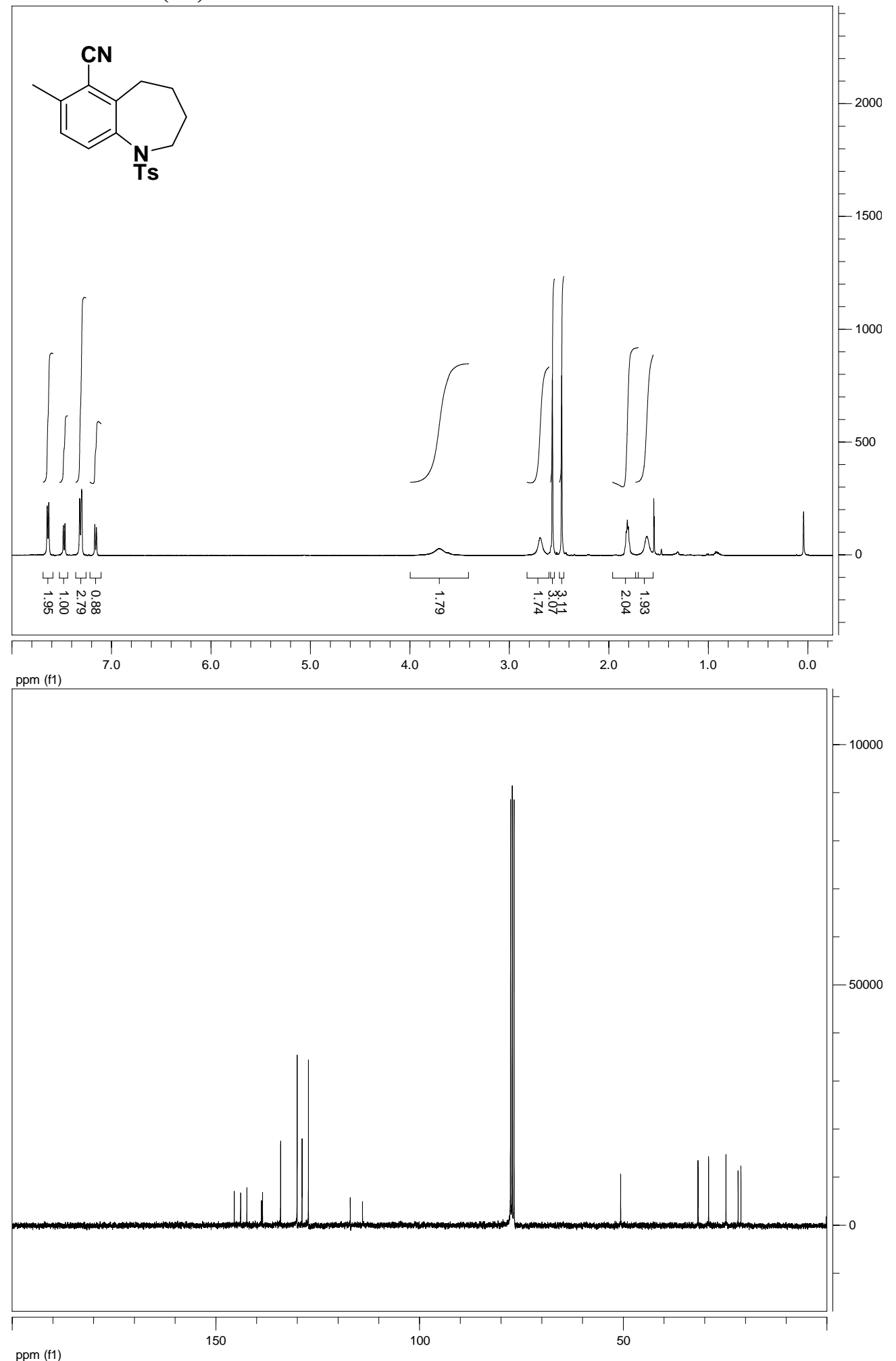


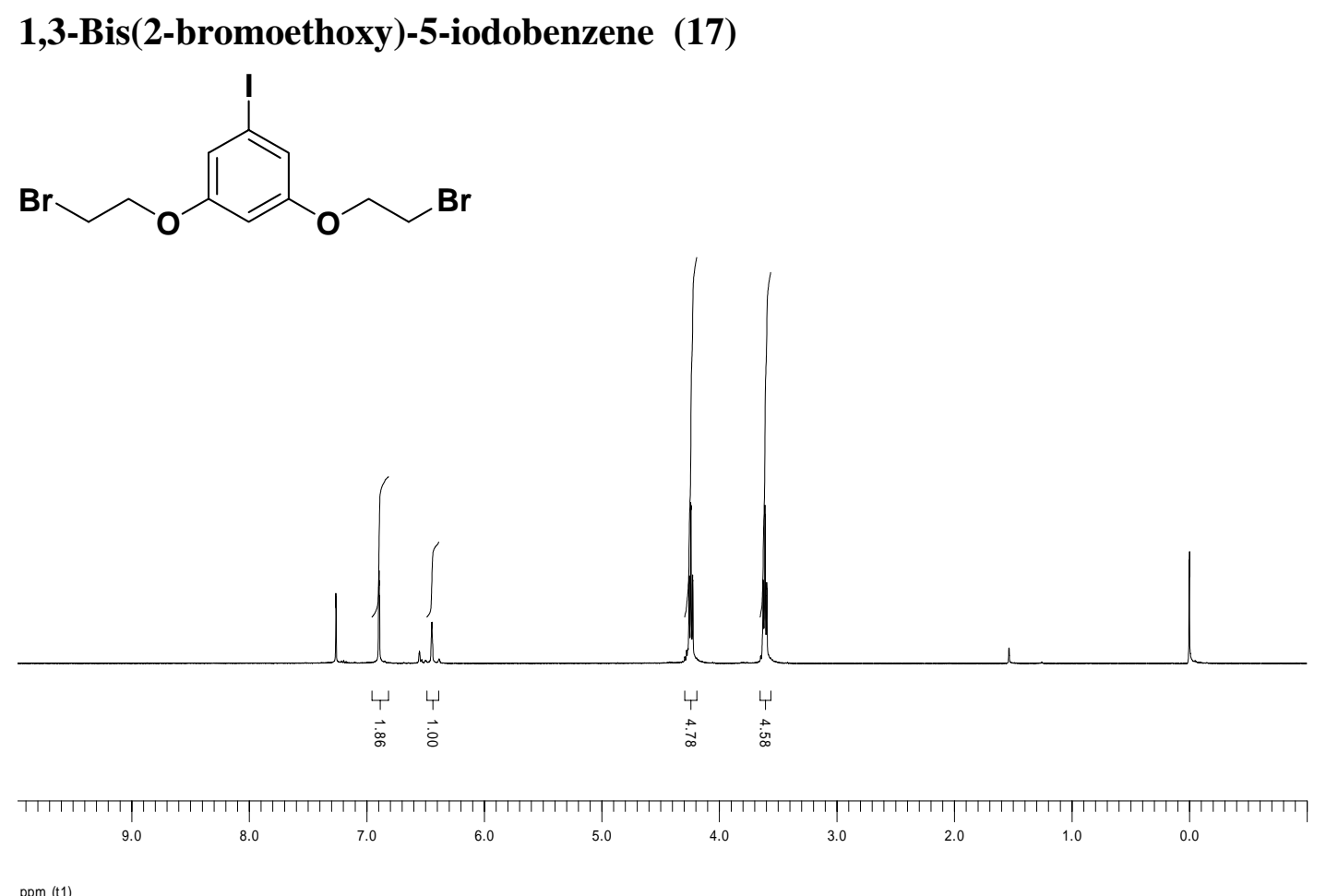

ppm (t1)

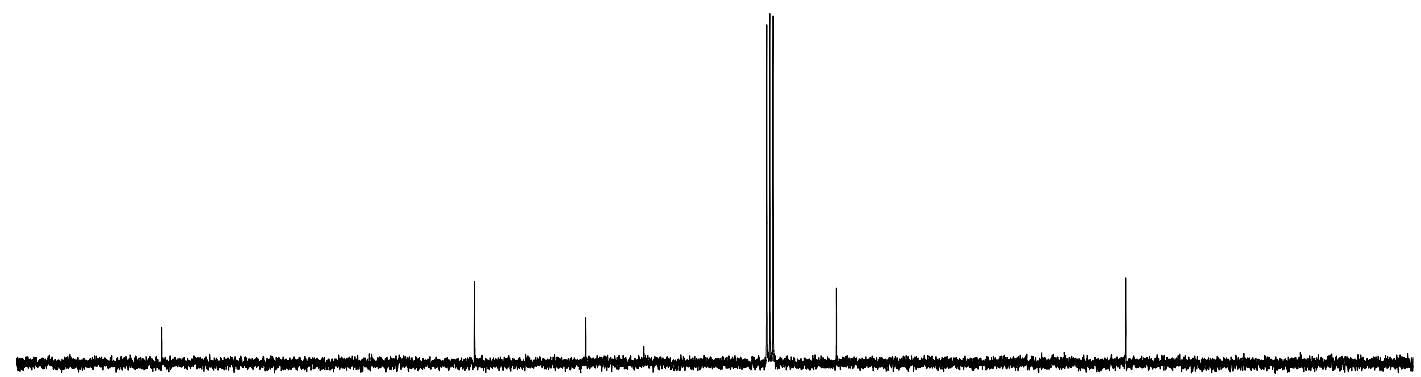

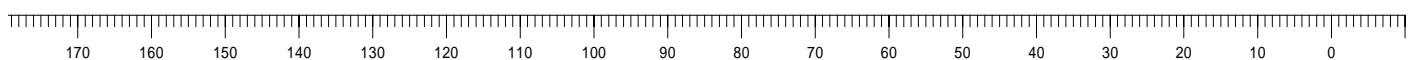
$\mathrm{ppm}(\mathrm{t} 1)$ 
2,3,5,6-Tetrahydro-benzo[1,2-b;5,4-b']difuran-4-carbonitrile (18)
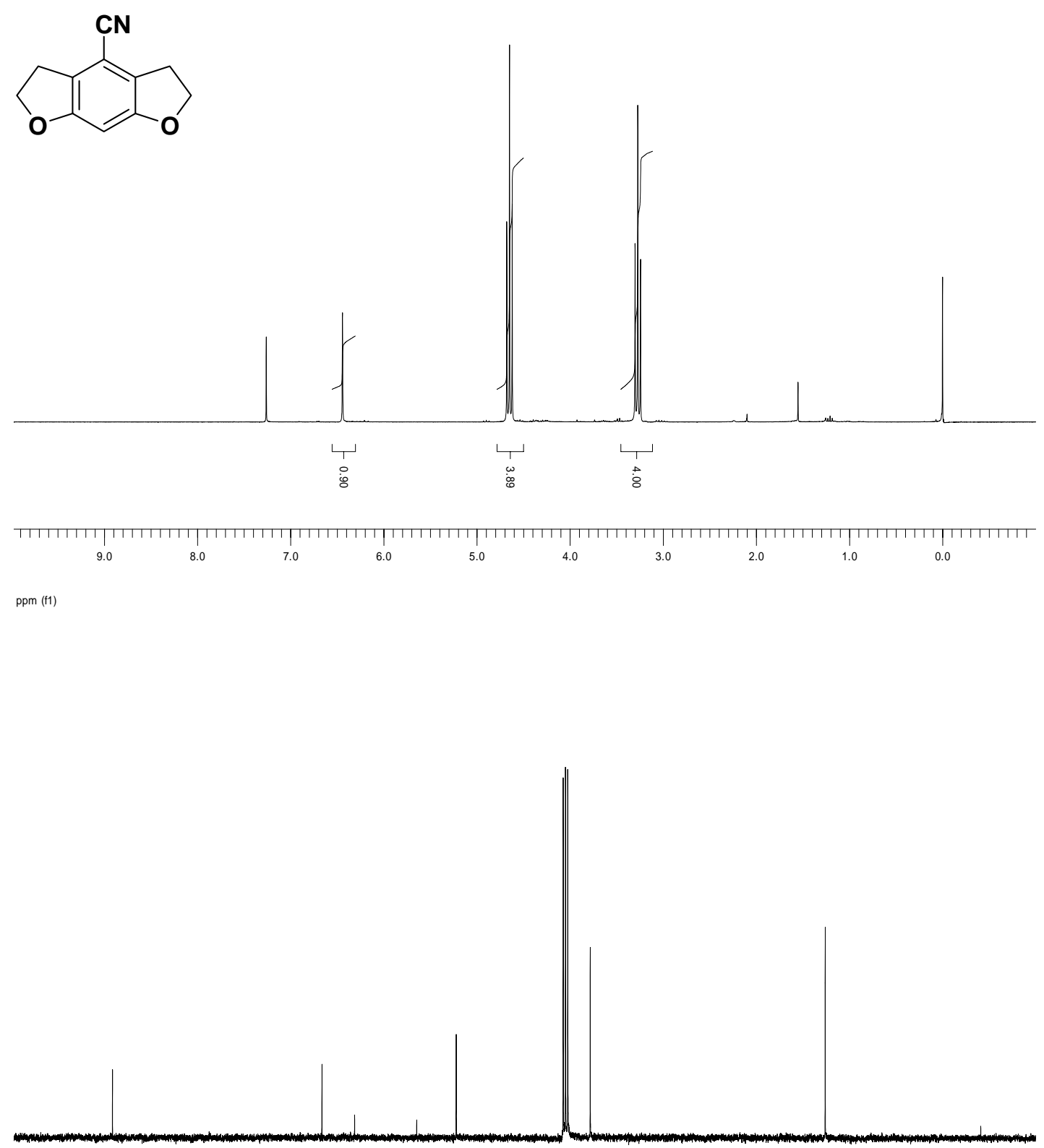

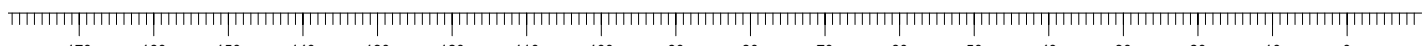
$\begin{array}{llllllllllllllllll}170 & 160 & 150 & 140 & 130 & 120 & 110 & 100 & 90 & 80 & 70 & 60 & 50 & 40 & 30 & 20 & 10 & 0\end{array}$

ppm (t1) 

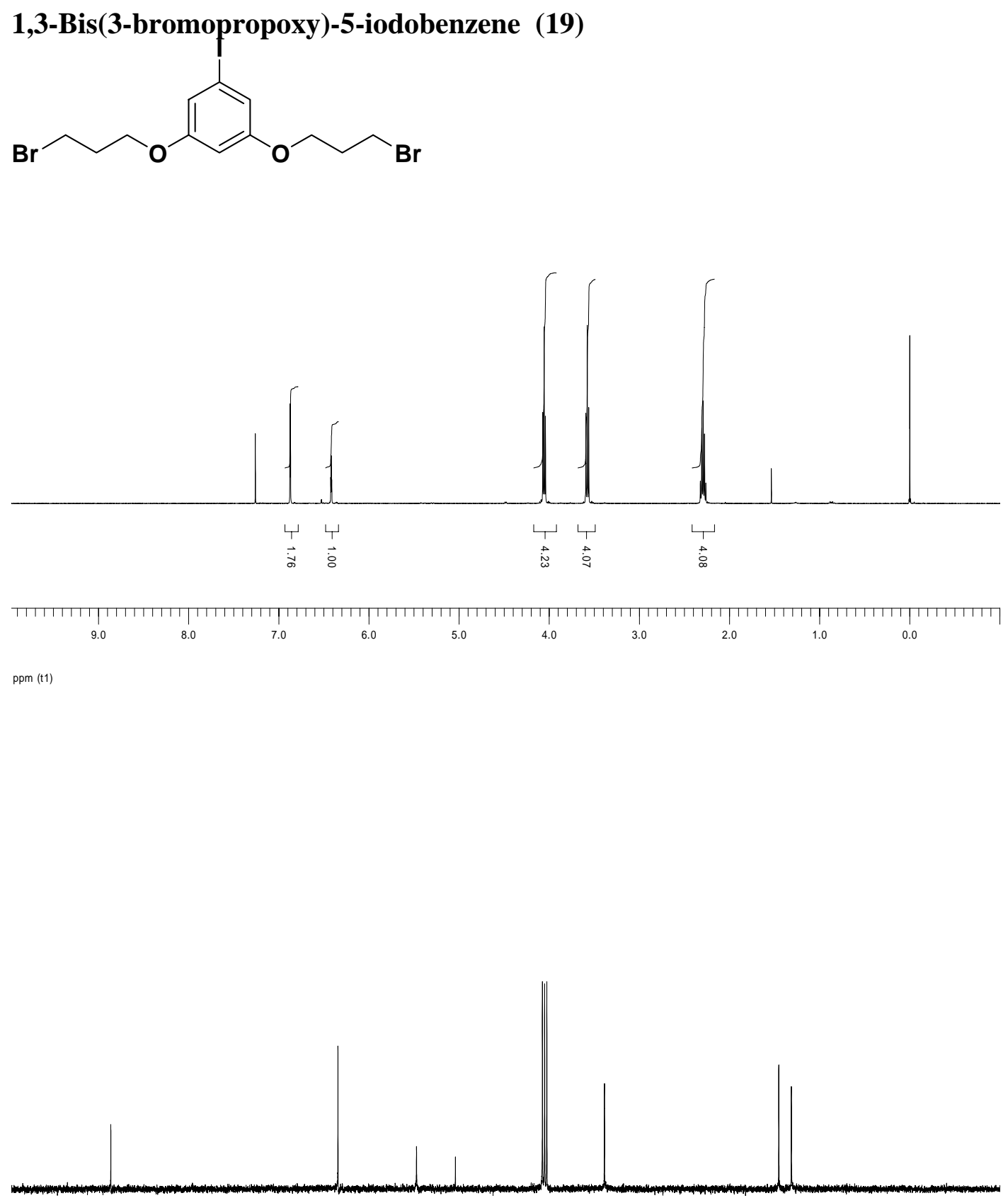

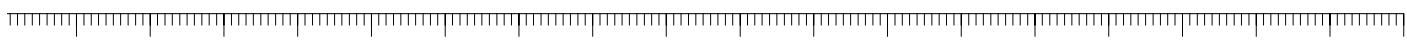

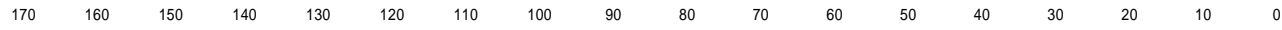

ppm (11) 
3,4,7,8-Tetrahydro-2H,6H-pyrano[3,2-g]chromene-5-carbonitrile (20)

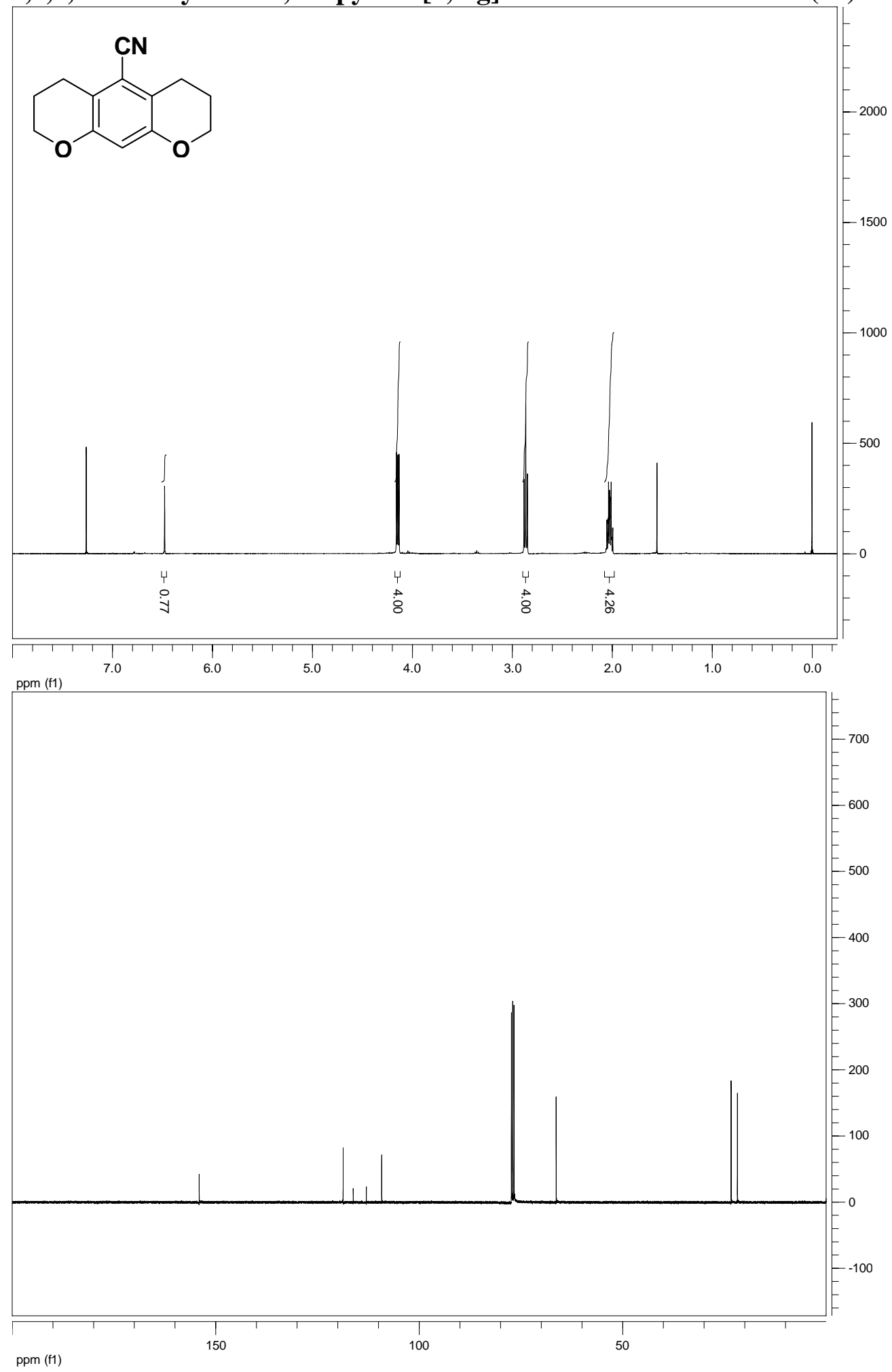



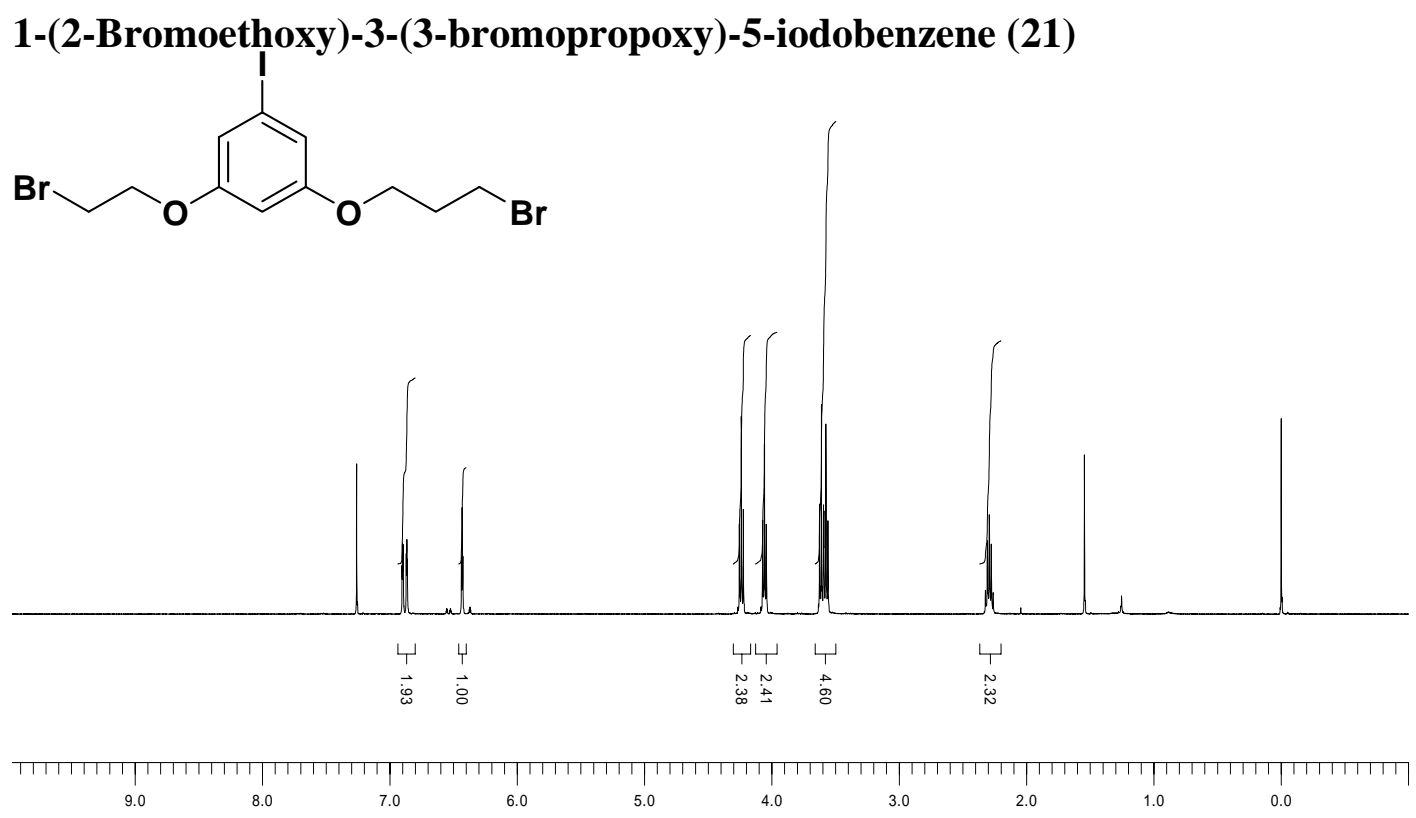

ppm (11)

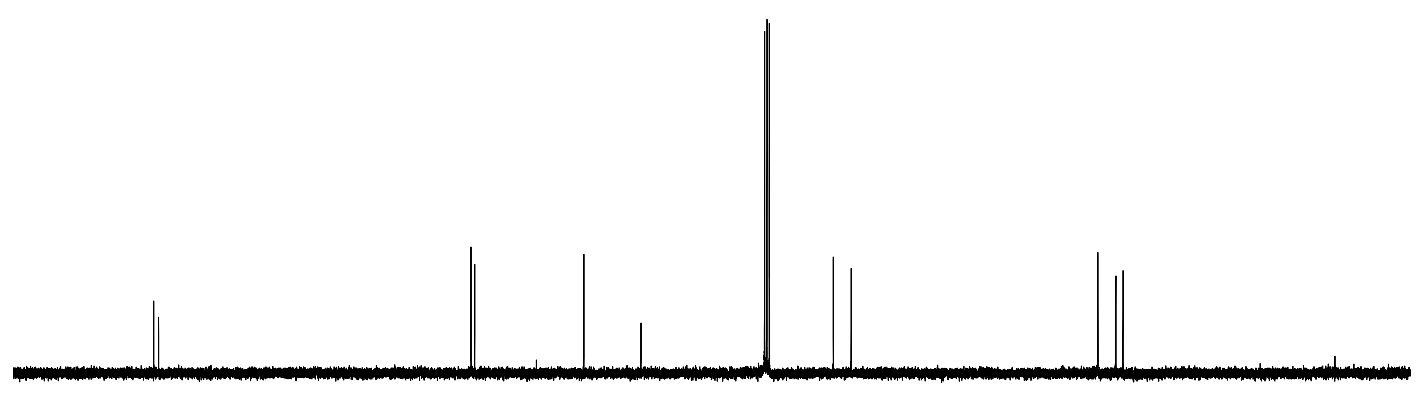

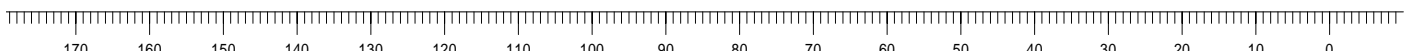
ppm (11) 
2,3,6,7-Tetrahydro-5H-furo[3,2-g]chromene-4-carbonitrile (22)
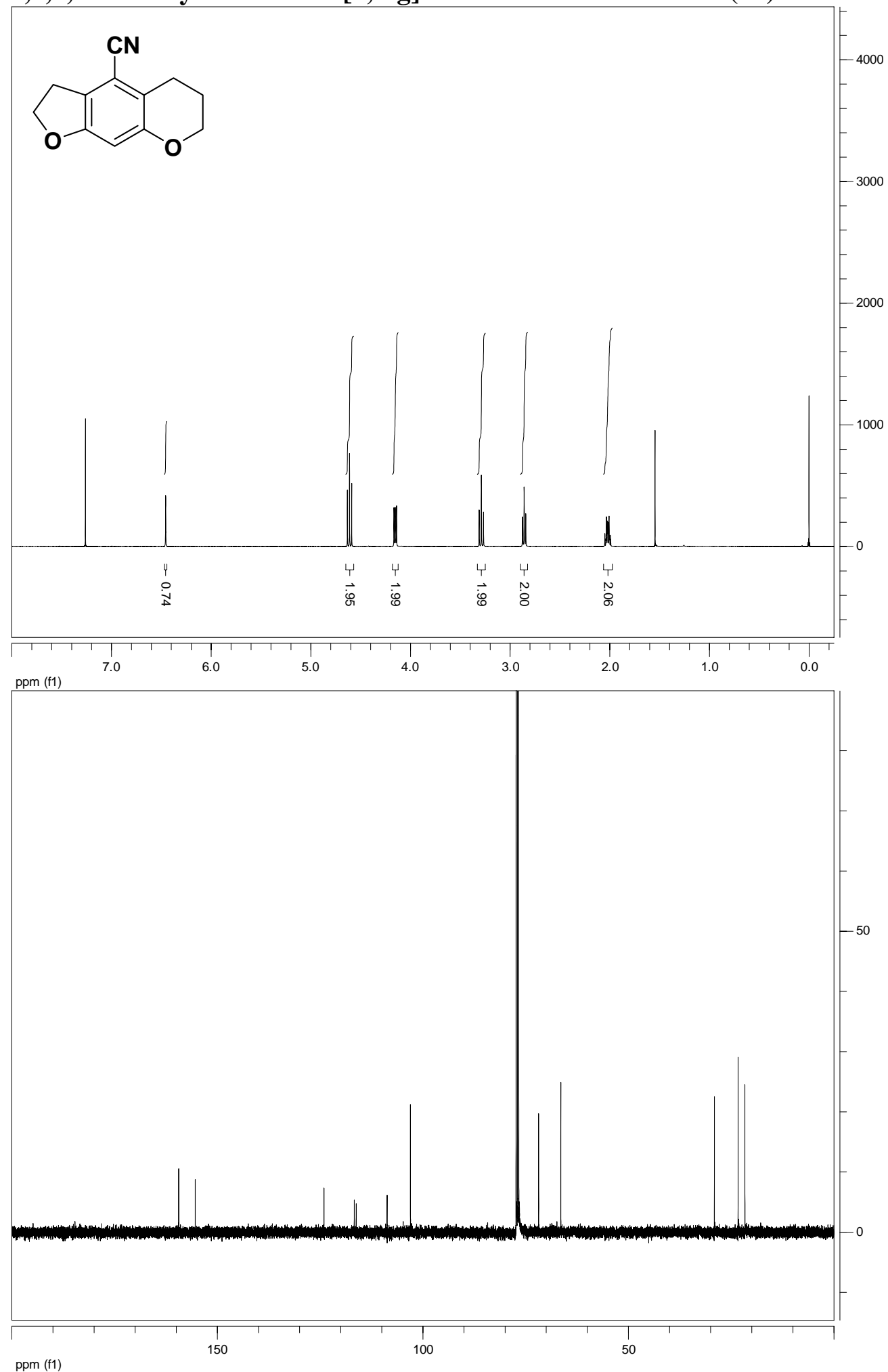


\section{1,3-Bis(2-bromoethoxy)-5-iodo-2-methoxybenzene (23)}<smiles>COc1c(OCCBr)cc(I)cc1OCCBr</smiles>
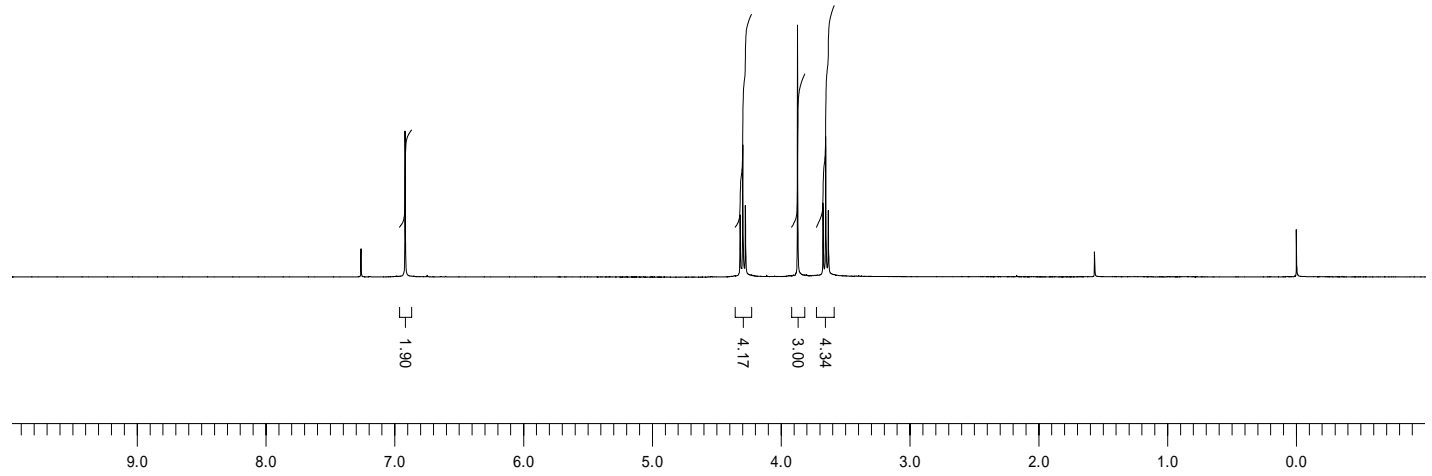

ppm (11)

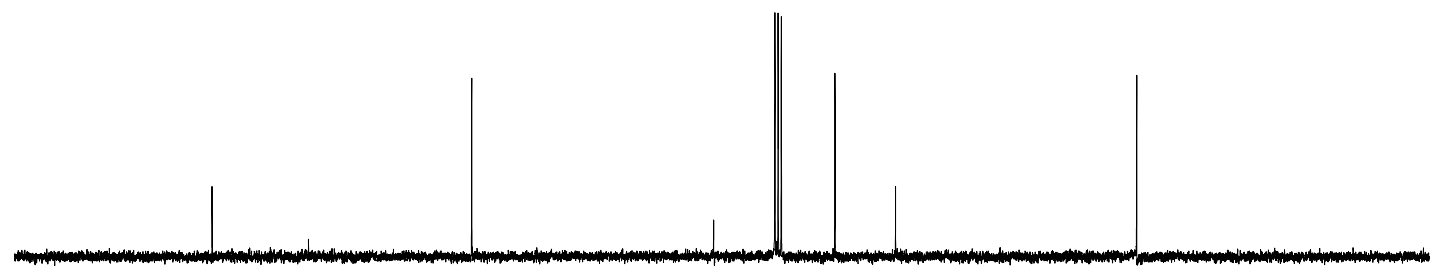

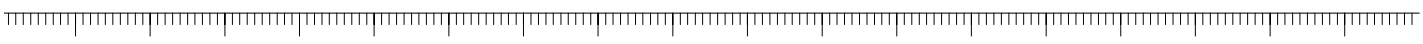

ppm (t1) 
8-Methoxy-2,3,5,6-tetrahydro-benzo[1,2-b;5,4-b']difuran-4-carbonitrile (24)
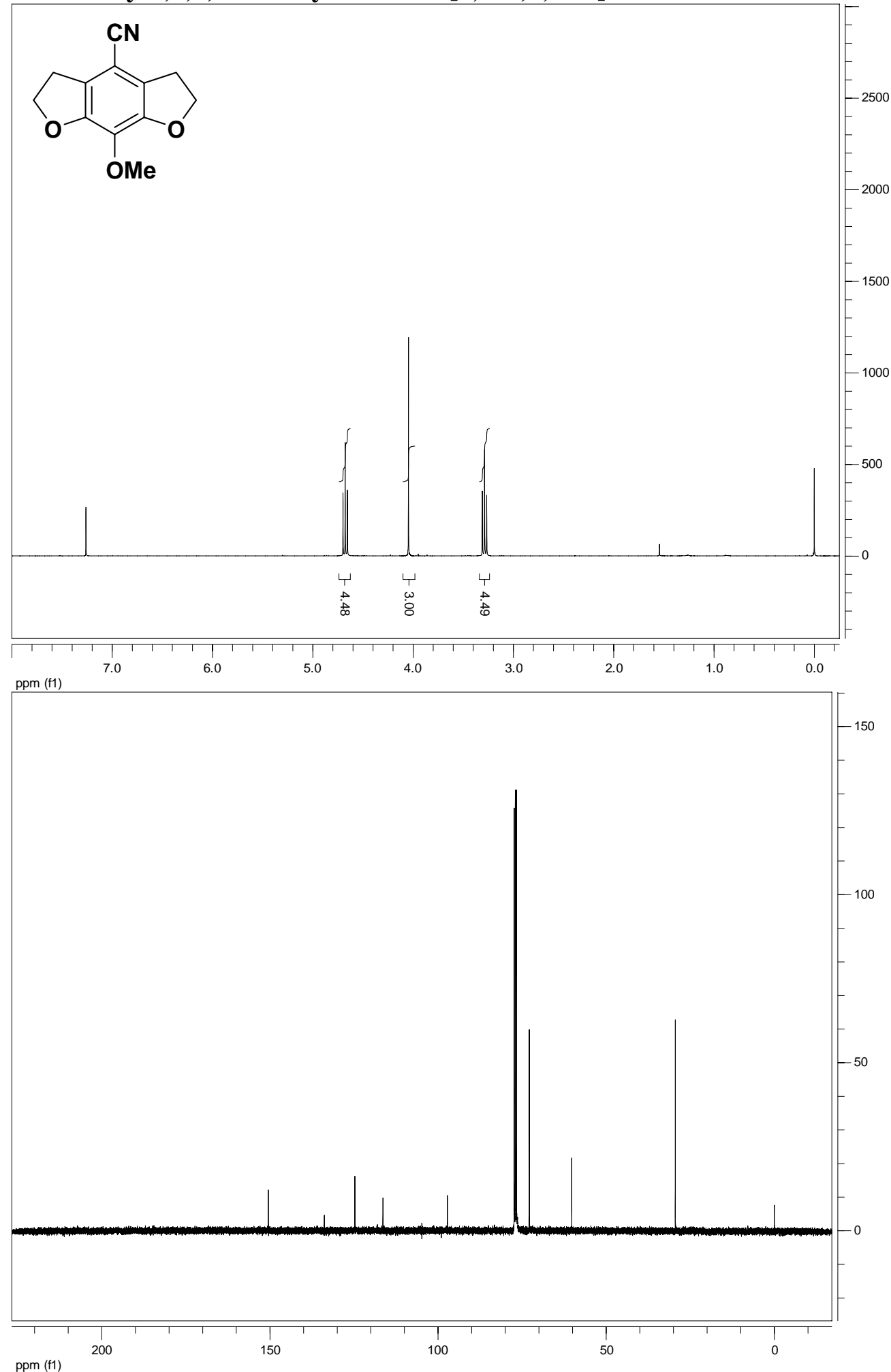
2,3,5,6-Tetrahydro-benzo[1,2-b;5,4-b']difuran-4-ylmethyl-ammonium; chloride (25)
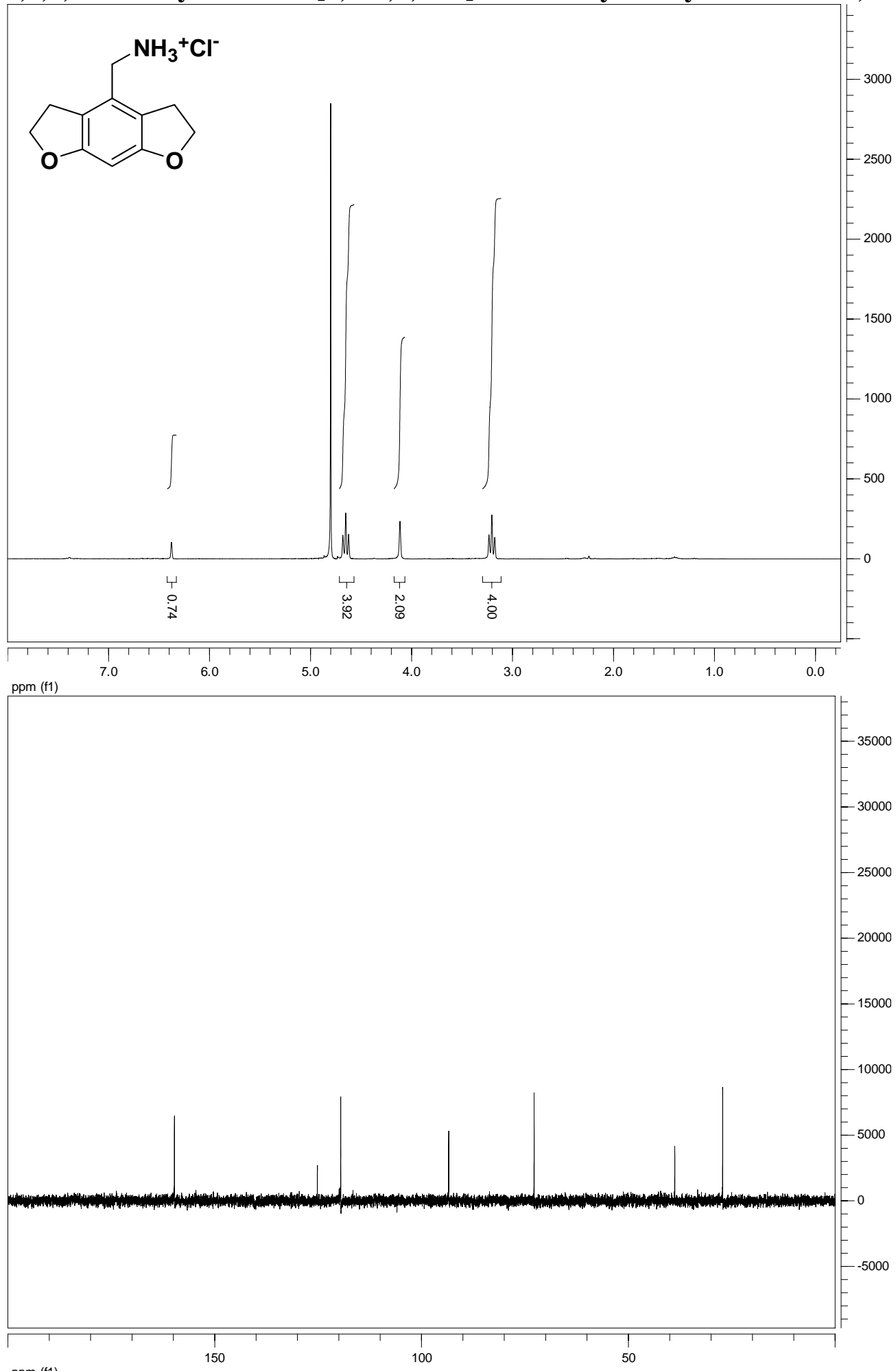

ppm (f1) 
2,3,5,6-Tetrahydro-benzo[1,2-b;5,4-b']difuran-4-carboxylic acid amide (26)
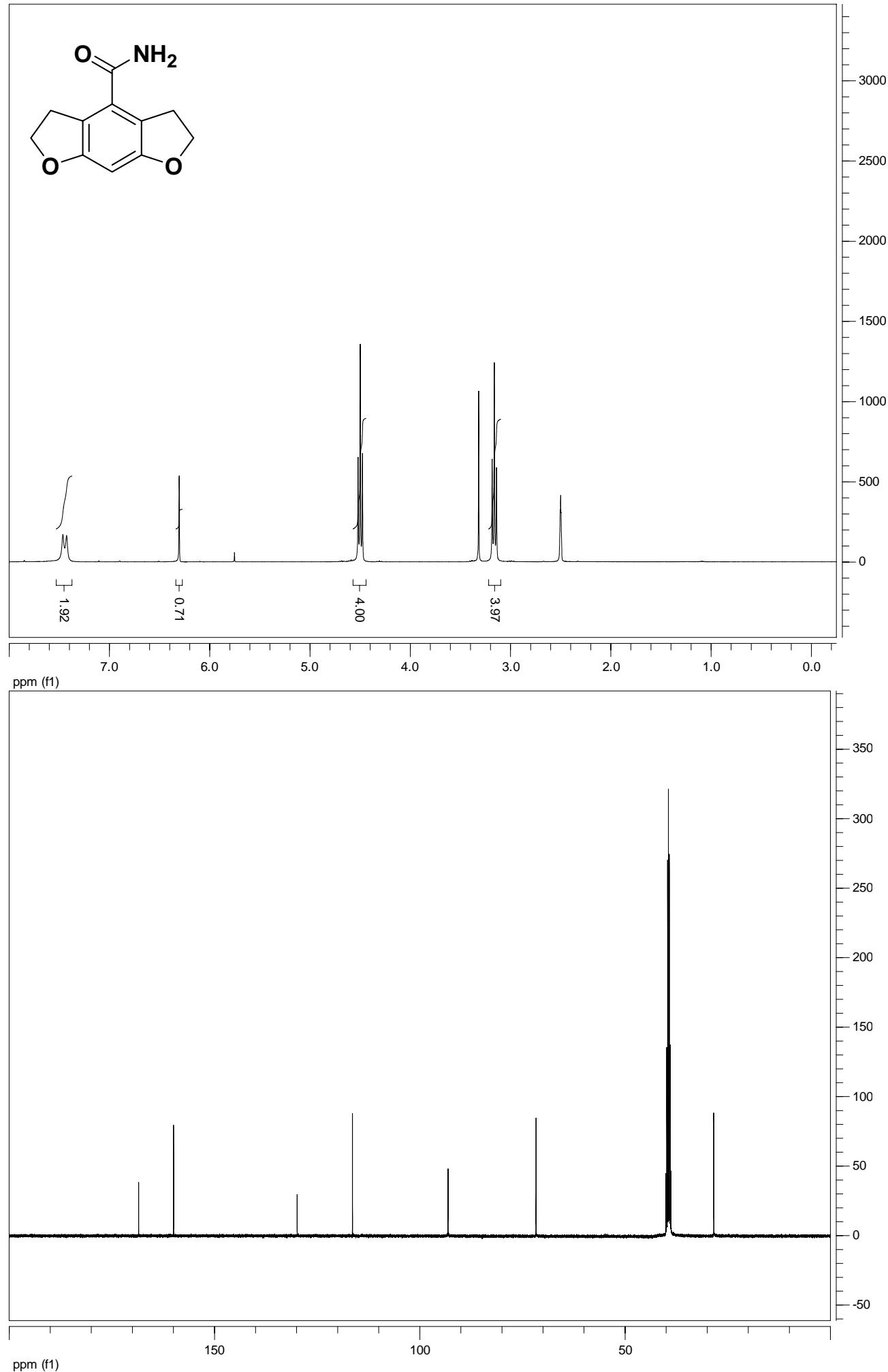
5-(2,3,5,6-Tetrahydro-benzo[1,2-b;5,4-b']difuran-4-yl)-1H-tetrazole (27)
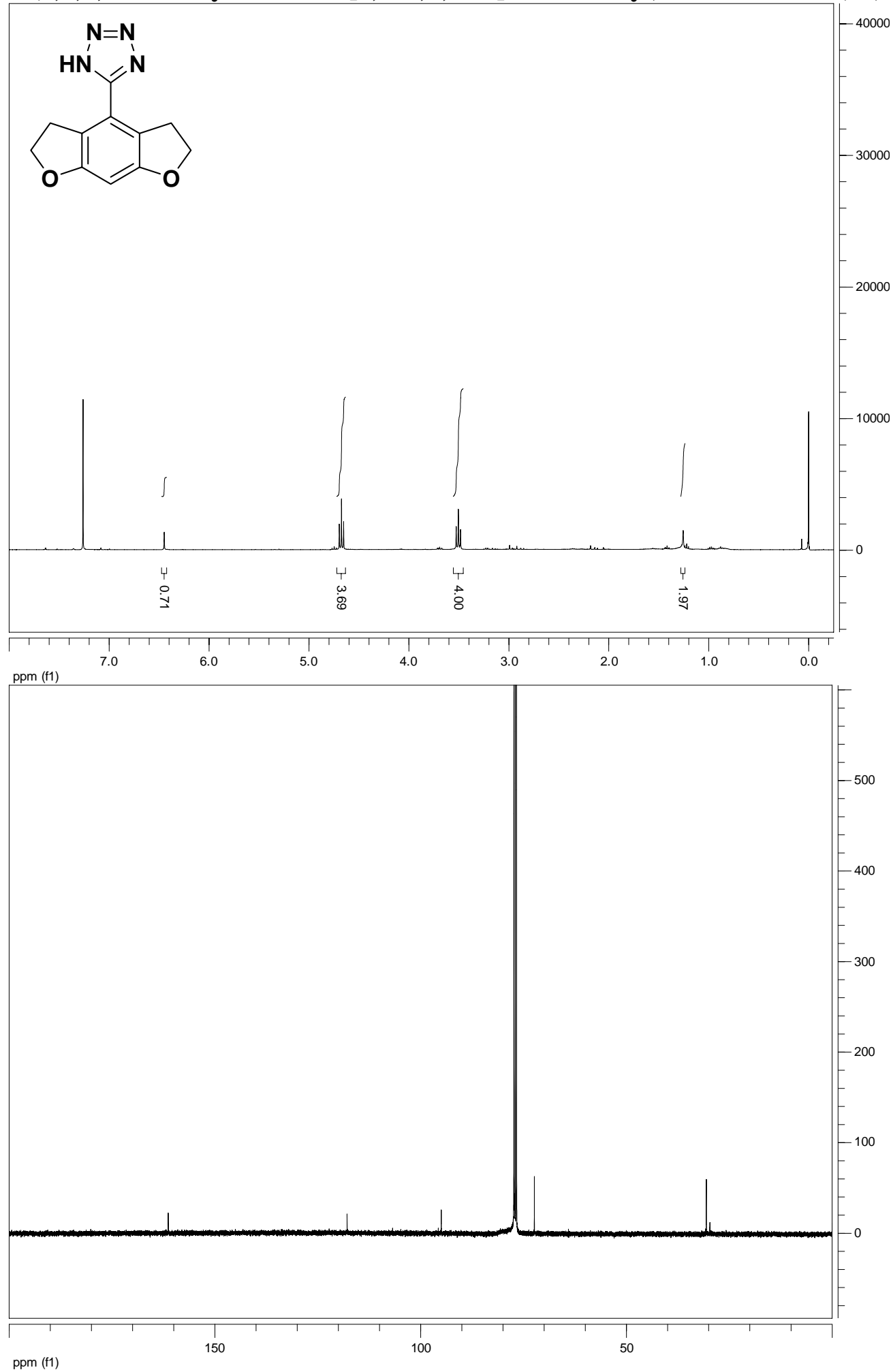
$N$-(3-Bromo-4-methyl-phenyl)-4-methyl-benzenesulfonamide (28)
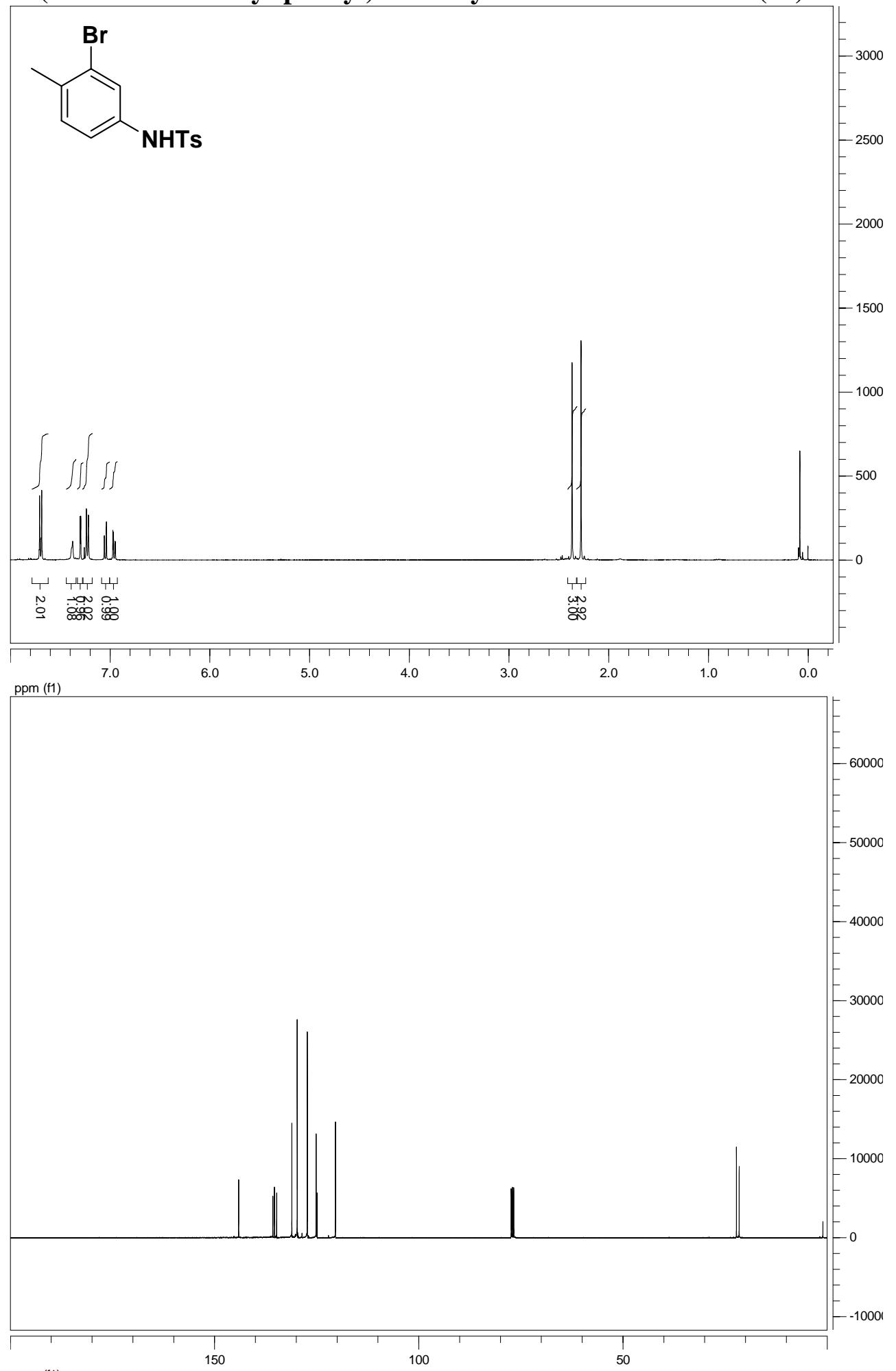

ppm (11) 


\section{1-Iodo-3,5-dimethoxybenzene (29)}<smiles>COc1cc(I)cc(OC)c1</smiles>
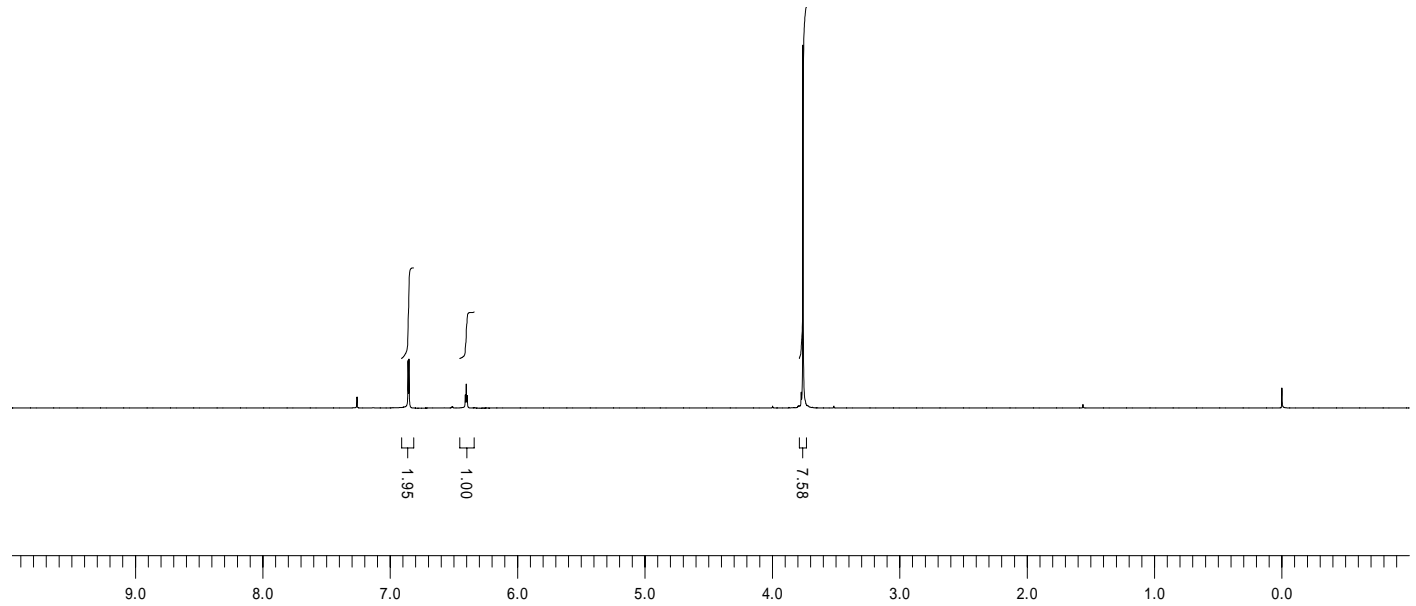

ppm (t1)

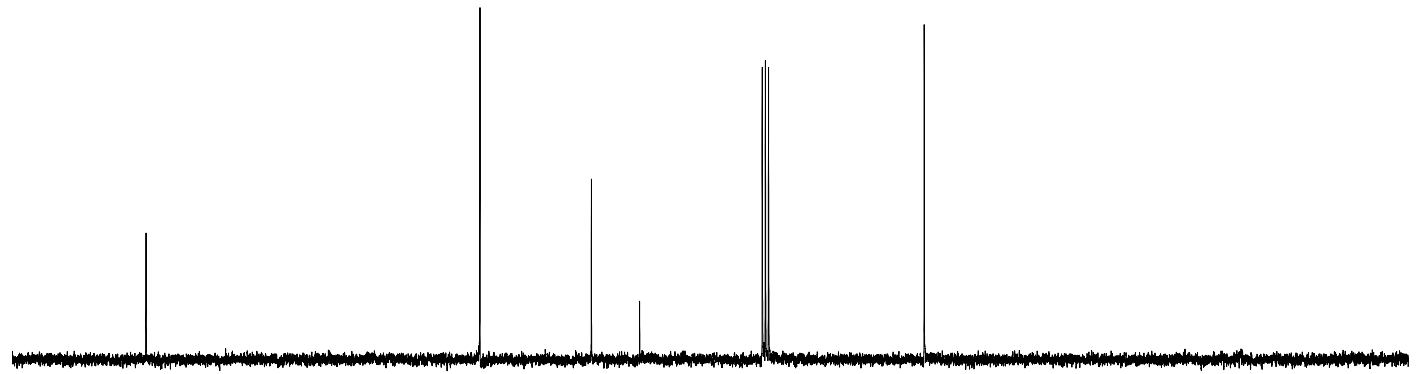

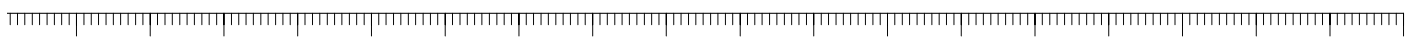

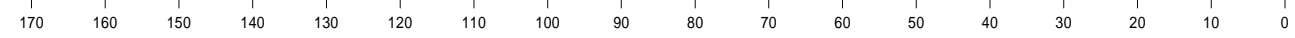

ppm (t1) 
5-Iodobenzene-1,3-diol (30)<smiles>Oc1cc(O)cc(I)c1</smiles>

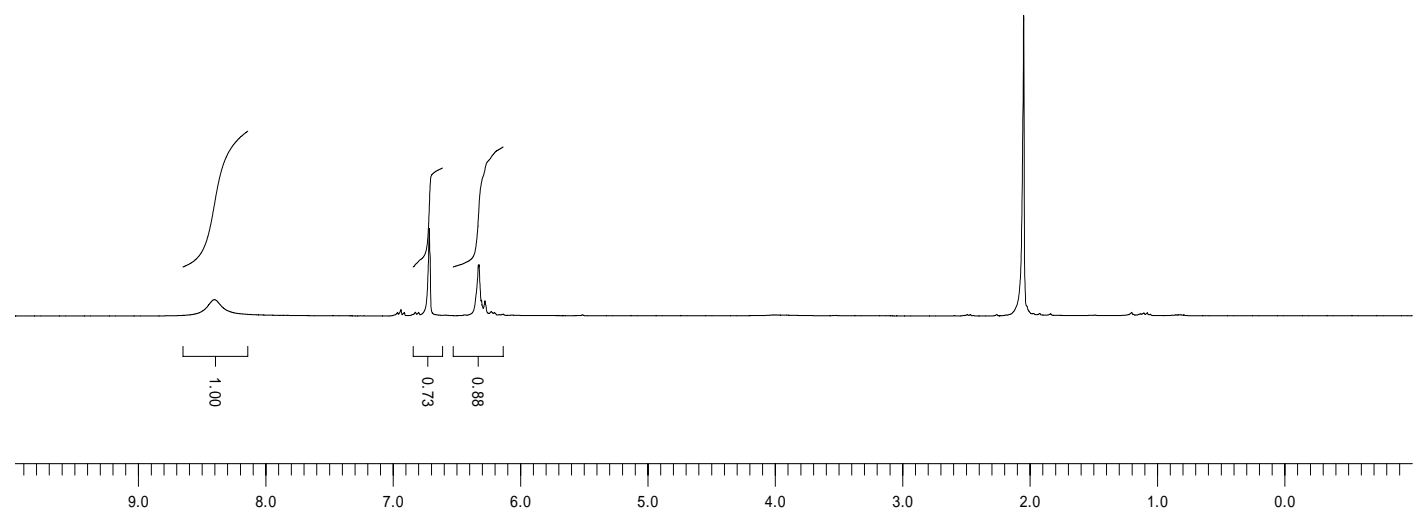

ppm (11)

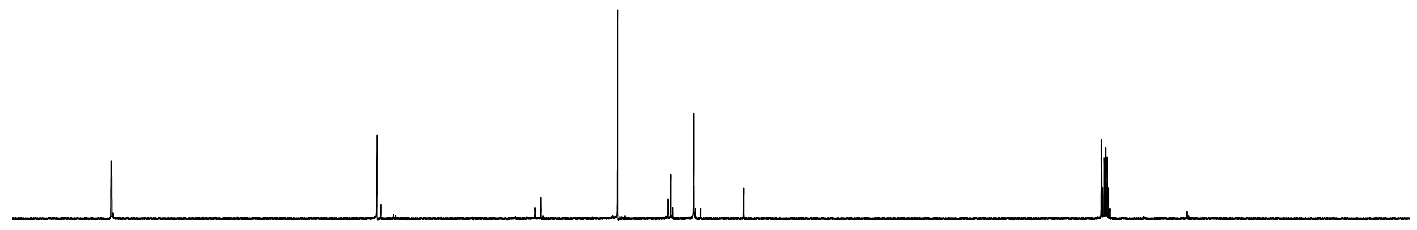

ताप |

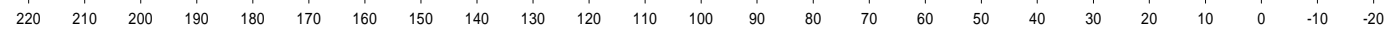
ppm (11) 


\section{3-(2-Bromoethoxy)-5-iodophenol (31)}<smiles>Oc1cc(I)cc(OCCBr)c1</smiles>
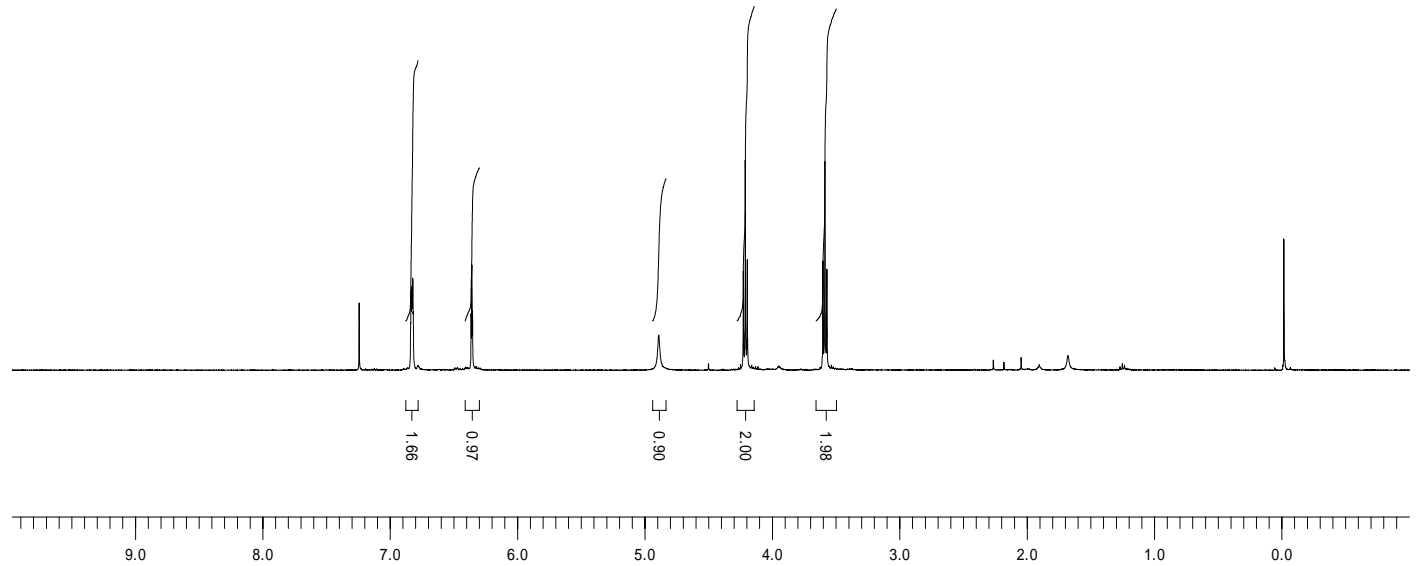

$\mathrm{ppm}(\mathrm{t} 1)$

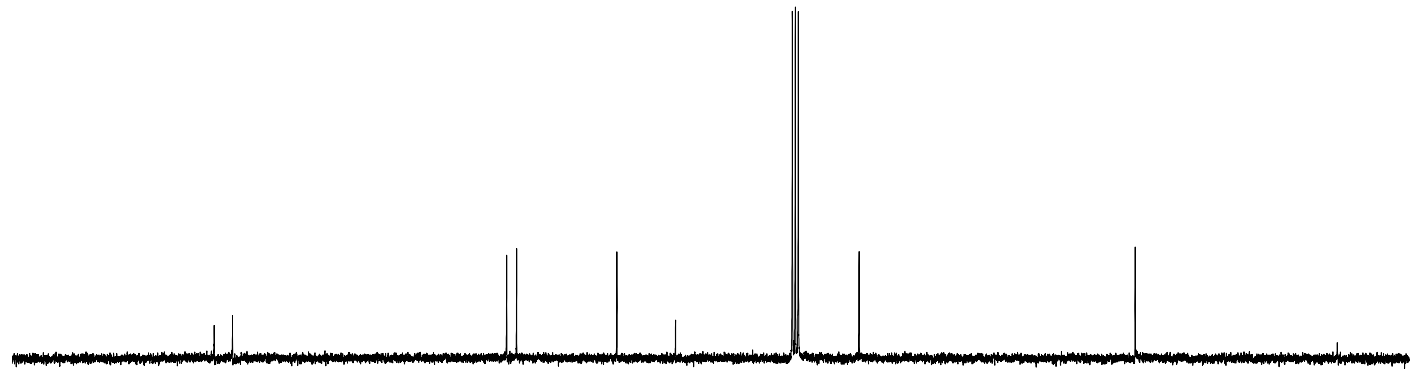

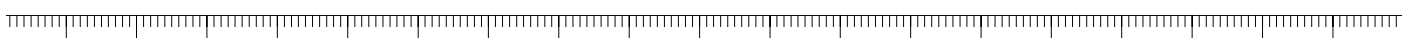

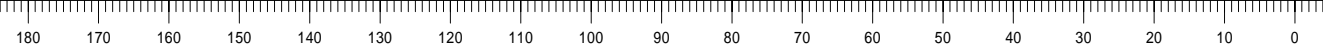
ppm (11) 


\section{1,3,5-Triiodo-2-methoxybenzene (32)}<smiles>COc1c(I)cc(I)cc1I</smiles>
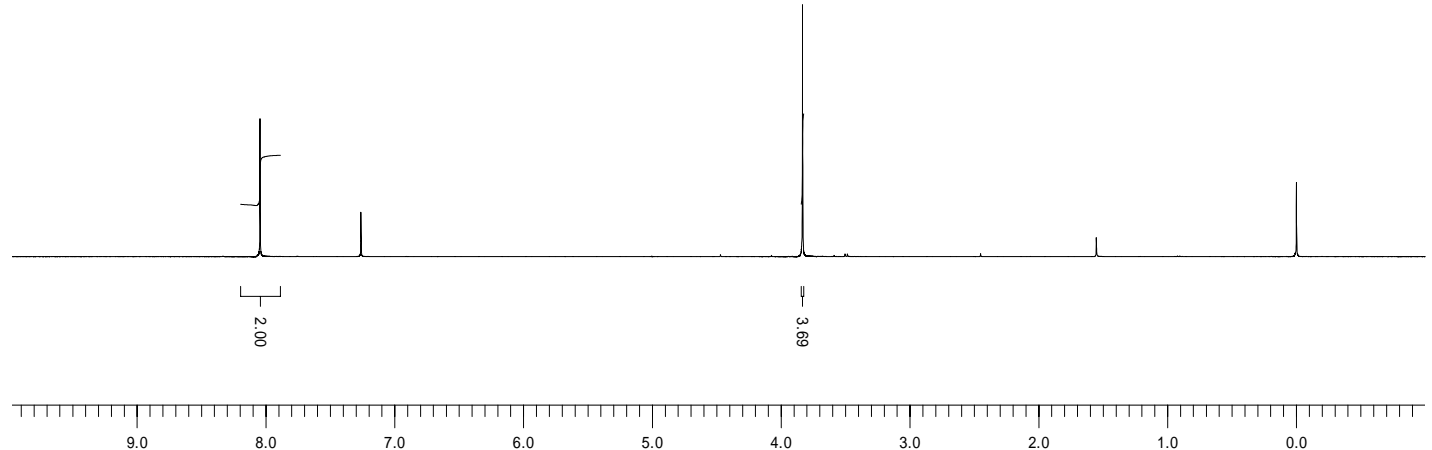

$\mathrm{ppm}(\mathrm{t} 1)$

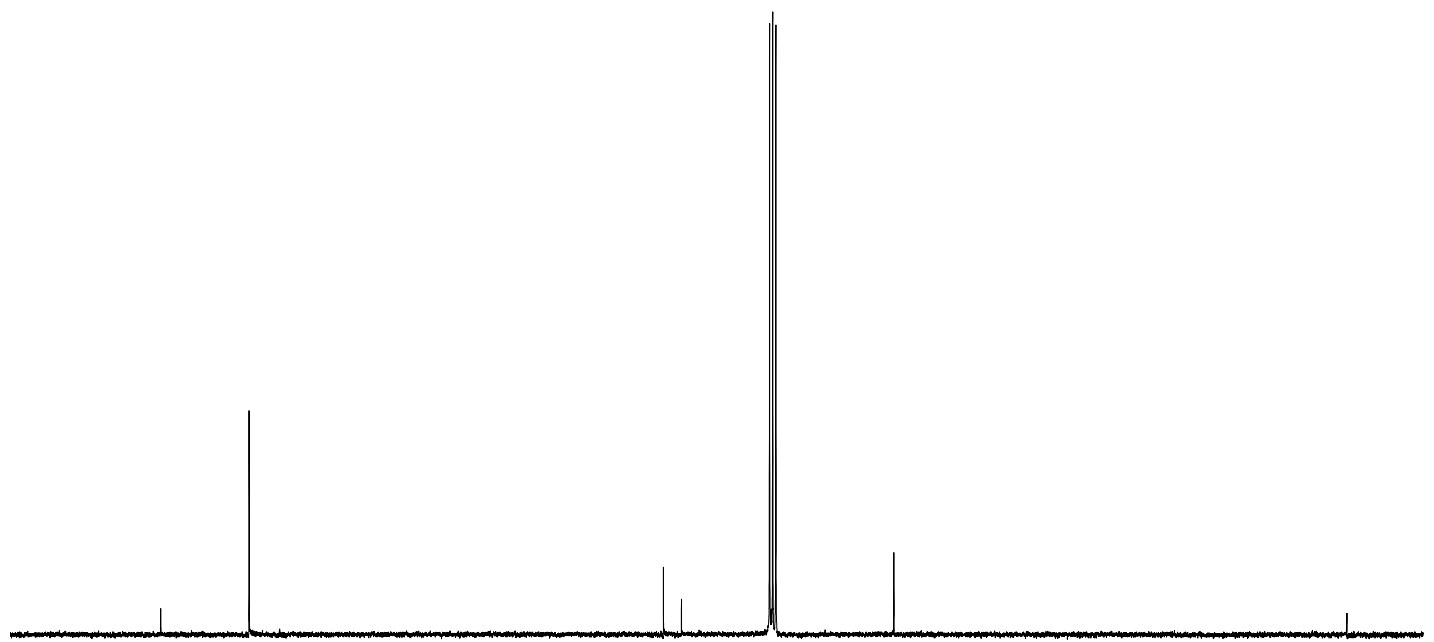

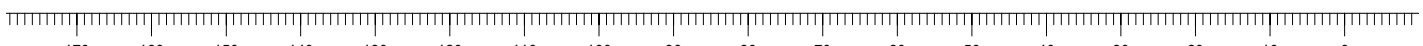

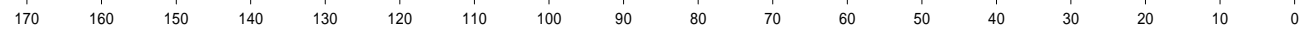

$\mathrm{ppm}(\mathrm{t} 1)$ 
5-Iodo-2-methoxybenzene-1,3-diol (33)<smiles>COc1c(O)cc(I)cc1O</smiles>
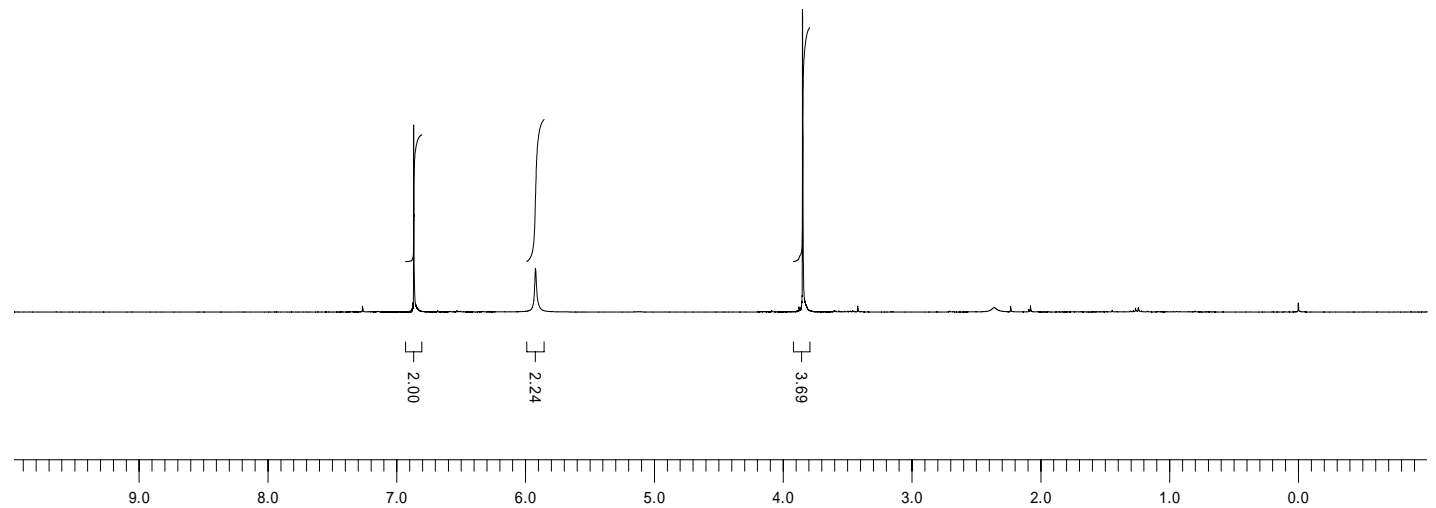

ppm (11)

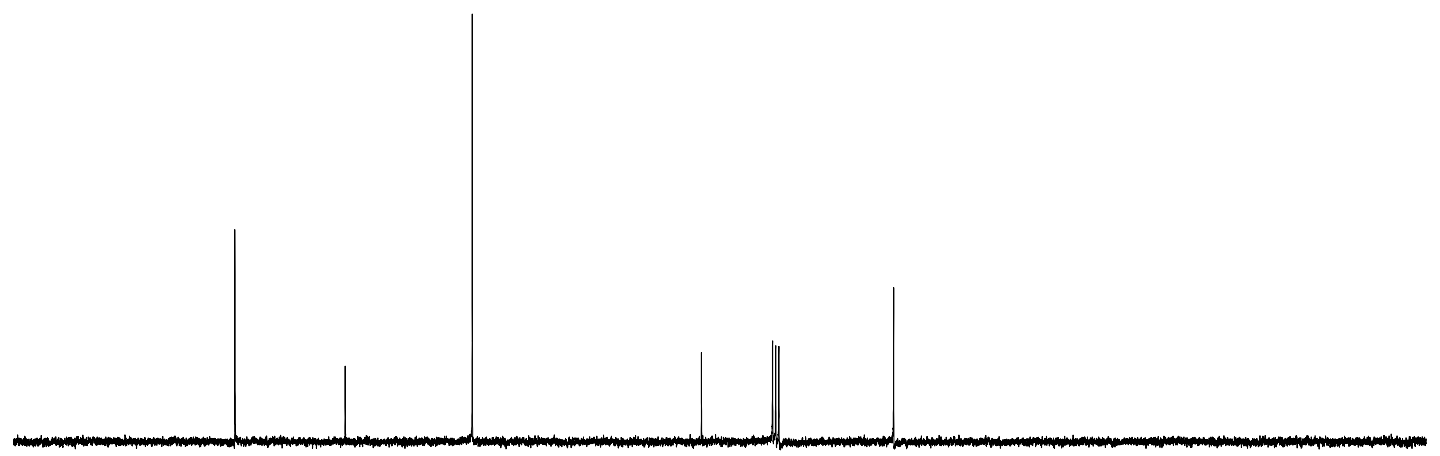

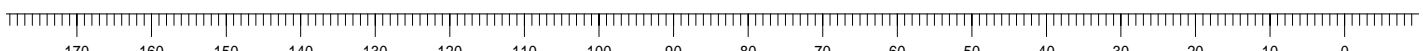
ppm (11) 